\title{
F-AREA ACID/CAUSTIC BASIN GROUNDWATER MONITORING REPORT (U)
}

\section{SECOND QUARTER 1994}

\section{Publication Date: September 1994}

Authorized Derivative Classifier:

UNCLASSIFIED

Does Not Contain Unclassified Controlled Nuclear Information

Westinghouse Savannah River Company Savannah River Site

Aiken, SC 29808 


\section{DISCLAIMER}

This report was prepared as an account of work sponsored by an agency of the United States Government. Neither the United States Government nor any agency thereof, nor any of their employees, makes any warranty, express or implied, or assumes any legal liability or responsibility for the accuracy, completeness, or usefulness of any information, apparatus, product, or process disclosed, or represents that its use would not infringe privately owned rights. Reference herein to any specific commercial product, process, or service by trade name, trademark, manufacturer, or otherwise does not necessarily constitute or imply its endorsement, recommendation, or favoring by the United States Government or any agency thereof. The views and opinions of authors expressed herein do not necessarily state or reflect those of the United States Government or any agency thereof.

This report has been reproduced directly from the best available copy.

Available to DOE and DOE contractors from the Office of Scientific and Technical Information, P.O. Box 62, Oak Ridge, TN 37831; prices available from (615) 576-8401.

Available to the public from the National Technical Information Service, U.S. Department of Commerce, 5285 Port Royal Road, Springfield, VA 22161. 


\section{F-AREA ACID/CAUSTIC BASIN GROUNDWATER MONITORING REPORT (U)}

\section{SECOND QUARTER 1994}

\section{Publication Date: September 1994}

Authorized Derivative Classifier:

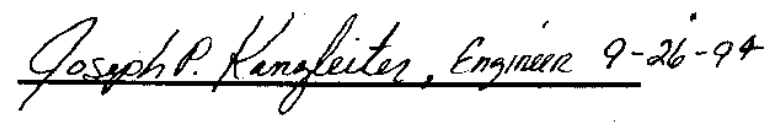

UNCLASSIFIED

Does Not Contain Unclassified

Controlled Nuclear Information

Westinghouse Savannah River Company

Savannah River Site

Aiken, SC 29808 
WSRC-TR-94-0347

Unclassified

\section{F-AREA ACID/CAUSTIC BASIN GROUNDWATER MONITORING REPORT (U)}

\section{SECOND QUARTER 1994}

Publication Date: September 1994

\section{Key Words}

aluminum

gross alpha

iron

manganese

total organic halogens

Westinghouse Savannah River Company

Savannah River Site

Aiken, SC 29808

Prepared for the U.S. Department of Energy under Control Contract No. DE-AC09-89SR18035 


\section{Abstract}

During second quarter 1994, samples from the FAC monitoring wells at the F-Area Acid/Caustic Basin were collected and analyzed for herbicides/pesticides, indicator parameters, metals, nitrate, radionuclide indicators, volatile organic compounds, and other constituents. Piezometer FAC 5P and monitoring well FAC 6 were dry and could not be sampled.

Analytical results that exceeded final Primary Drinking Water Standards (PDWS), other Savannah River Site (SRS) Flag 2 criteria, or the SRS turbidity standard of 50 NTU during the quarter were as follows: gross alpha exceeded the final PDWS and aluminum, iron, manganese, and total organic halogens exceeded the SRS Flag 2 criteria in one or more of the FAC wells. Turbidity
exceeded the SRS standard in well FAC 3 .

Groundwater flow direction and rate in the water table beneath the F-Area Acid/Caustic Basin
were similar to past quarters. 
THIS PAGE LEFT BLANK INTENTIONALLY. 


\section{Contents}

Page

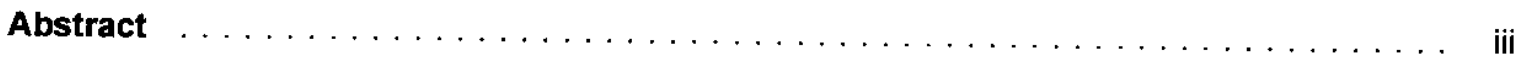

List of Figures $\ldots \ldots \ldots \ldots \ldots \ldots \ldots \ldots \ldots \ldots \ldots \ldots \ldots \ldots \ldots$

List of Tables $\ldots \ldots \ldots \ldots \ldots \ldots \ldots \ldots \ldots \ldots \ldots \ldots \ldots \ldots \ldots \ldots$

Executive Summary $\ldots \ldots \ldots \ldots \ldots \ldots \ldots \ldots \ldots \ldots \ldots \ldots \ldots$

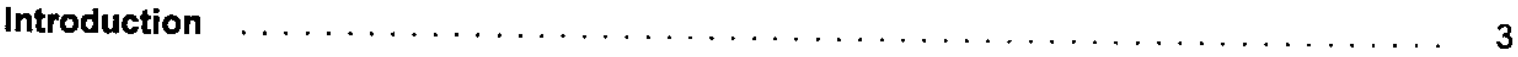

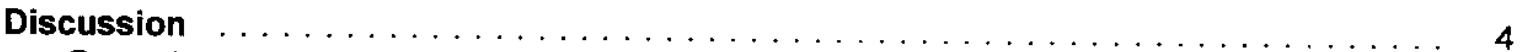

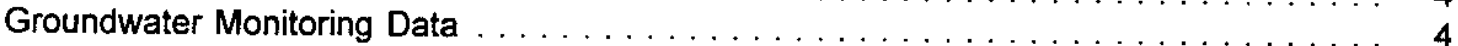

Analytical Results Exceeding Standards $\ldots \ldots \ldots \ldots \ldots \ldots \ldots \ldots \ldots \ldots$

Turbidity Results Exceeding Standards $\ldots \ldots \ldots \ldots \ldots \ldots \ldots \ldots \ldots \ldots \ldots \ldots \ldots$

Water Elevations, Flow Directions, and Flow Rates $\ldots \ldots \ldots \ldots \ldots \ldots \ldots \ldots \ldots$

Results for Upgradient vs. Downgradient Wells $\ldots \ldots \ldots \ldots \ldots \ldots \ldots \ldots$

Conclusions $\ldots \ldots \ldots \ldots \ldots \ldots \ldots \ldots \ldots \ldots \ldots \ldots \ldots \ldots \ldots \ldots$

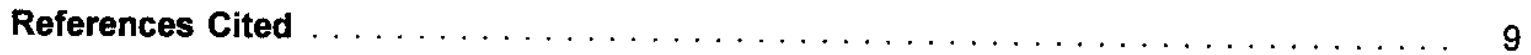

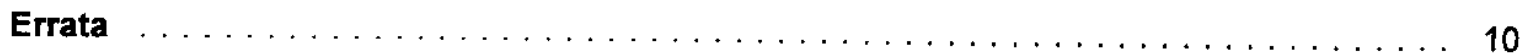

Appendix A-Final Primary Drinking Water Standards $\ldots \ldots \ldots \ldots \ldots \ldots \ldots$

Appendix B-Flagging Criteria $\ldots \ldots \ldots \ldots \ldots \ldots \ldots \ldots \ldots \ldots \ldots \ldots \ldots$

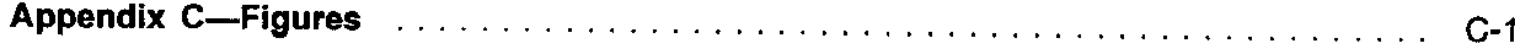

Appendix D-Groundwater Monitoring Results Tables $\ldots \ldots \ldots \ldots \ldots \ldots \ldots$

Appendix E-Data Quality/Useability Assessment $\ldots \ldots \ldots \ldots \ldots \ldots \ldots \ldots$ E-1 .

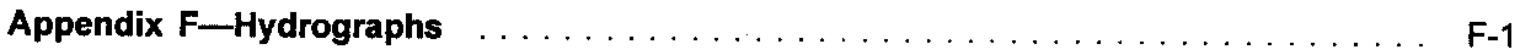




\section{List of Figures}

1.

1. Location of the F-Area Acid/Caustic Basin at the Savannah River Site . . . . . . C C-3

2. Location of Groundwater Monitoring Wells and Piezometer Wells at the F-Area
Acid/Caustic Basin

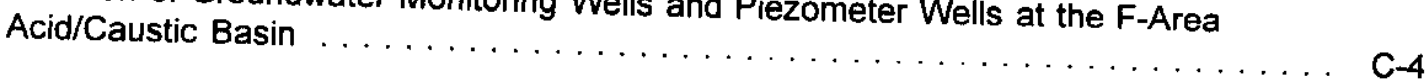

3. Piezometric Surface Map of the Water Table at the F-Area Acid/Caustic Basin,
Monitoring Well Data

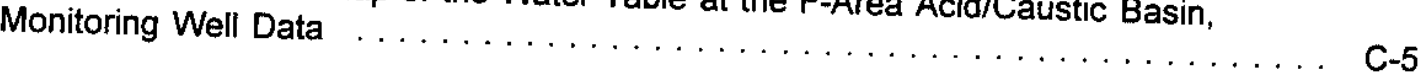
4. Water-Elevation Contour Map of the Water Table at the F-Area Acid/Caustic Basin,
Piezometer Data ... . . . .

\section{List of Tables}

1. Maximum Results for Constituents Exceeding Final Primary Drinking Water
Standards

2. Maximum Results for Constituents Exceeding Other Flag 2 Criteria or the SRS
Turbidity Standard....

3. Groundwater Monitoring Results for Individual Wells 


\section{Executive Summary}

The six monitoring wells and piezometer FAC 5P at the F-Area Acid/Caustic Basin are scheduled for sampling quarterly as part of the Savannah River Site (SRS) Groundwater Monitoring Program and to comply with South Carolina Hazardous Waste Management Regulations.

During second quarter 1994, groundwater from successfully sampled monitoring wells was analyzed for herbicides/pesticides, indicator parameters, metals, nitrate, radionuclide indicators, volatile organic compounds, and other constituents. Piezometer FAC 5P and monitoring well FAC 6 were dry and were not sampled. Monitoring results that exceeded final Primary Drinking Water Standards (PDWS), other SRS Flag 2 criteria, or the SRS turbidity standard are the focus
of this report.

Gross alpha was the only constituent exceeding final PDWS in the FAC wells during second quarter; it was elevated in one well. Aluminum, iron, manganese, total organic halogens, and turbidity exceeded SRS Flag 2 criteria in one or more of the FAC wells. Aluminum, iron, total organic halogens, and turbidity were elevated in the upgradient wells FAC 3 and 5 .

Groundwater flow direction and rate in the water table beneath the F-Area Acid/Caustic Basin
were similar to past quarters. 
THIS PAGE LEFT BLANK INTENTIONALLY. 


\section{Introduction}

The F-Area Acid/Caustic Basin is located east of F Area at the Savannah River Site (SRS) (Figure 1, Appendix C) on a slope that leads to an unnamed tributary of Upper Three Runs Creek. The following description outlines important events in the history of the F-Area Acid/Caustic Basin.

- The F-Area Acid/Caustic Basin, constructed in the early 1950s, is an unlined earthen pit that received dilute sulfuric acid and sodium hydroxide solutions and other wastes from several areas within SRS. The basin provided mixing and neutralization of the dilute solutions before their discharge to nearby streams (Heffner and Exploration Resources, 1991).

- The basin remained in service until new neutralization facilities became operational in 1982 (Heffner and Exploration Resources, 1991).

- Wells FAC 1, 2, 3, and 4 were installed at the basin between August 1983 and July 1984 (EPD/EMS, 1994).

- Under the terms of a consent decree signed May 26, 1988, by the U.S. District Court (Civif Action 1:85-2583-6, District of South Carolina, Aiken Division), the basin became subject to requirements of Subtitle $\mathrm{C}$ of the Resource Conservation and Recovery Act (RCRA), the South Carolina Hazardous Waste Management Regulations (SCHWMR), and associated regulations on June 1, 1988.

- The FAC monitoring wells were reevaluated during the summer of 1988 to ensure compliance with SCHWMR. As part of this compliance effort, wells FAC 5,6, 7, and 8 were installed at the basin during third quarter 1988 (EPD/EMS, 1994).

- Wells FAC 1 and 2 were abandoned in March 1989 (EPD/EMS, 1994) because they were dry and were not included in the RCRA monitoring program.

- The revised Groundwater Quality Assessment Plan for the F-Area Acid/Caustic Basin (WSRC, 1991), submitted to the South Carolina Department of Health and Environmental Control (SCDHEC) on April 30, 1991, proposed the installation of six permanent piezometers to provide additional water-elevation data that would help define the groundwater flow direction and assess the current monitoring well network as required by SCHWMR.

- The six piezometers were installed in early 1992 (EPD/EMS, 1994) (Figure 2, Appendix C).

- During first quarter 1993, piezometer FAC 5P was added to the sampling schedule.

Currently, the Environmental Protection Department/Environmental Monitoring Section (EPD/EMS) samples monitoring wells FAC 3, 4, 5, 6, 7, and 8 and piezometer FAC 5P (Figure 2, Appendix $C$ ) each quarter as part of the SRS Groundwater Monitoring Program. The Environmental Restoration Department provides a quarterly report describing the monitoring results to SCDHEC in compliance with SCHWMR. 


\section{Discussion}

\section{Groundwater Monitoring Data}

The EPD/EMS groundwater sampling procedure (EPD/EMS, 1992) requires evacuation of a minimum of two well volumes and stabilization of $\mathrm{pH}$, specific conductance, and turbidity prior to sample collection. Stability is established when a minimum of three successive measurements, taken within a given time period, are within a specified tolerance range. If a well pumps dry before two well volumes are purged or before stabilization is achieved, it must be revisited within 24 hours for the data to be considered the result of a single sampling event. On the second visit within 24 hours, samples are taken without purging or measuring stability; thus, these samples may not be representative of the groundwater quality.

During second quarter 1994, samples from the five successfully sampled monitoring wells at the F-Area Acid/Caustic Basin were analyzed for herbicides/pesticides, indicator parameters, metals, nitrate, radionuclide indicators, volatile organic compounds, and other constituents. This report describes results that equaled or exceeded the Safe Drinking Water Act final Primary Drinking Water Standards (PDWS) or drinking water screening levels, as established by the U.S. Environmental Protection Agency (EPA) (Appendix A); the South Carolina final PDWS for lead (Appen$\operatorname{dix}$ A); SRS flagging criteria that are based on final and proposed PDWS, Secondary Drinking Water Standards, and method detection limits (Appendix B); or the SRS turbidity standard. Constituent levels that equaled or exceeded the final PDWS, screening levels, or other Flag 2 criteria are described as exceeding or above standards or as elevated.

The final PDWS for individual analytes presented in Appendix A may not always match the SRS flagging criteria presented in Appendix B. The final PDWS generally are used in this compliance report as guidelines to meet regulatory requirements; the flagging criteria are used by EPD/EMS to identify relative levels of constituents in the groundwater and as guides for scheduling ground-
water monitoring.

\section{Analytical Results Exceeding Standards}

Results for analytes that exceeded the final PDWS (Appendix A) during second quarter 1994 are summarized in Table 1 (Appendix D). Gross alpha activity exceeded the final PDWS in monitoring well FAC 3 , with a maximum activity of $2.3 E+01 \mathrm{pCi} / \mathrm{L}$. No other constituent exceeded final PDWS this quarter.

Constituents that exceeded other flagging criteria (Appendix B) during second quarter are summarized in Table 2 (Appendix D). Aluminum exceeded its Flag 2 criterion in all FAC wells sampled this quarter, with a maximum concentration of $3,130 \mu \mathrm{g} / \mathrm{L}$ in well FAC 3 . Iron exceeded its Flag 2 criterion in wells FAC 3,5 , and 8 , with a maximum concentration of $11,100 \mu \mathrm{g} / \mathrm{L}$ in well FAC 3. Manganese exceeded its Flag 2 criterion in well FAC 4 , at $519 \mu \mathrm{g} / \mathrm{L}$. Total organic halogens was elevated in wells FAC 3 and 5 , with a maximum concentration of $93 \mu \mathrm{g} / \mathrm{L}$ in well FAC 5.

Table 3 (Appendix D) presents all of the results for individual wells and indicates the analytical laboratories that conducted the analyses, the dilution factors used in the analyses, and the 
analyses that received modifiers (which help identify laboratory accuracy and precision) or that exceeded the EPA-approved holding times during second quarter 1994. Constituent results in Table 3 that appear to equal the final PDWS but are not marked in the $D$ column (exceeded final PDWS or screening level) are below the final PDWS in the database. Database results, the results that are compared to the final PDWS, are entered with more significant digits than the results given in this report. Apparent discrepancies are the result of the rounding of reported results.

Table 3 also lists the number of well volumes purged from each well during second quarter 1994 and provides a statement that describes incomplete or unsuccessful sampling events. Well FAC 5P pumped dry before any field parameters were collected. Well FAC 6 went dry before samples were collected and yielded depth to water only. Wells FAC $3,5,7$, and 8 failed to produce two well volumes before samples were collected; thus, these wells may not have produced representative groundwater samples.

Appendix $D$ provides definitions of the abbreviations and the modifiers used in the results tables as well as descriptions of holding times, data rounding, and data qualification practices.

Appendix $E$ provides a general assessment of the quality and useability of the data provided by EPD/EMS.

\section{Turbidity Results Exceeding Standards}

A value of 5 nephelometric turbidity units (NTU), established by EPA (1986) as a general standard for acceptability of groundwater samples, is considered unrealistic for monitoring wells at SRS. Gass (1989) has documented turbidity measurements as high as 5,000 NTU from properly designed wells screened in poorly productive formations. During the 1989 RCRA Compliance Monitoring Evaluation Inspection, officials from EPA Region IV indicated that the SRS turbidity standard of 50 NTU is conservative. These officials also agreed that water-table wells in this area often correspond to nonaquifer formations, rendering development of these wells more difficult due to the low yield and high proportion of mobile fines typical of these formations (Bergren and Bennett, 1989).

During second quarter 1994, the laboratory-determined turbidity of samples from wells FAC 4,5 , 7 , and 8 was less than 5 NTU. Well FAC 3 exceeded the SRS turbidity standard with a value of 198 NTU (Tables 2 and 3, Appendix D). Wells FAC 5P and 6 purged insufficient water volumes to permit turbidity measurements.

\section{Water Elevations, Flow Directions, and Flow Rates}

Because most of the FAC monitoring wells are screened into and through a clay-confining layer (the tan clay) beneath the F-Area Acid/Caustic Basin, water-level measurements from the FAC monitoring wells do not provide a clear groundwater flow pattern and thus have made flow rate estimates uncertain. Piezometers screened above this clay layer were installed during 1992 to provide additional water elevations for more accurate estimates of groundwater flow direction and rate within the water table.

Figure 3 (Appendix C) was generated using only water elevations from the FAC monitoring wells, and Figure 4 (Appendix C) was generated using only water elevations from the FAC piezometers (FAC 1P through 6P). Using universal transverse Mercator coordinates, the groundwater flow direction derived from FAC monitoring well water elevations is west, whereas the direction derived using the FAC piezometers is northwest. 

The groundwater flow rate in the water table (Aquifer Zone $\| \mathrm{B}_{2}$ ) beneath the F-Area Acid/Caustic
Basin is estimated using the following equation:

$$
\text { Flow }(\mathrm{ft} / \text { day })=\frac{\text { Hydraulic Conductivity }(\mathrm{ft} / \text { day) }}{\text { Porosity (unitless) }} \times \frac{d h(\mathrm{ft})}{d l(\mathrm{ft})}
$$

A hydraulic conductivity constant of $10 \mathrm{ft} /$ day (Geraghty \& Miller, Inc., 1990) is a conservative estimate (i.e., the actual hydraulic conductivity should be somewhat less than $10 \mathrm{ft} /$ day). The and $d l$ is the length of the flow path to the (Killian et al., 1987); $d \mathrm{~h}$ is the difference in head, on the vertical gradient between wells, the of data points. For this reason, the estimation of the area under consideration, and the number an order of magnitude.

Flow rate estimates are calculated as follows: flow rate per day is calculated to two significant figures using the above equation. This value is then multiplied by 365 and rounded to two signif-
icant figures for the flow rate per year.

The flow rate estimate for groundwater in the water table beneath the F-Area Acid/Caustic Basin using water-level data from the FAC monitoring wells (see Figure 3, Appendix $C$ ) is as follows:

$$
\begin{aligned}
& \frac{10}{0.20} \times \frac{6}{30}=10 \mathrm{ft} / \text { day } \\
& 10 \mathrm{ft} / \text { day } \times 365 \text { days } \approx 3,700 \mathrm{ft} / \mathrm{yr}
\end{aligned}
$$

The flow rate estimate for groundwater in the water table beneath the F-Area Acid/Caustic Basin using water-level data from the FAC piezometers (see Figure 4, Appendix $C$ ) is as follows:

$$
\frac{10}{0.20} \times \frac{0.9}{130}=0.35 \mathrm{ft} / \mathrm{day}
$$

$$
0.35 \mathrm{ft} \text { day } \times 365 \text { days } \approx 130 \mathrm{ft} / \mathrm{yr}
$$

Estimated Horizontal Groundwater Flow Rate (ft/yr) in the Water Table beneath the
F-Area Acid/Caustic Basin

\begin{tabular}{|lllll|}
\hline Flow Rate & $3 \mathrm{Q} 93$ & $4 \mathrm{Q} 93$ & 1 Q94 & 2 Q94 \\
\hline Monitoring wells & 2,700 & 2,400 & 3,700 & 3,700 \\
Piezometers & 120 & 130 & 150 & 130 \\
\hline
\end{tabular}

Water elevations for the FAC piezometers probably represent fully unconfined conditions, while water elevations for FAC monitoring wells intersecting the clay layer may have equilibrated with hydrostatic head below the clay layer. As a result, water-table conditions determined by piezometer water elevations are more representative of true water-table conditions than those determined by water elevations obtained at monitoring wells.

As can be seen in Figures 3 and 4 (Appendix $C$ ), the monitoring wells display a greater gradient over a shorter distance than do the piezometers. This difference is due to the variation in 
screening of the monitoring wells through and into the tan clay. The monitoring wells essentially depict a variation in head resulting from different pressure gradients. Hydrographs for the FAC monitoring wells and the FAC piezometers are presented in
Appendix $F$.

\section{Results for Upgradient vs. Downgradient Wells}

Based on the groundwater flow direction determined from the piezometer data, wells FAC 3 and 5 are upgradient at the F-Area Acid/Caustic Basin, while well FAC 6 is downgradient.

During second quarter 1994, upgradient well FAC 3 contained gross alpha above the final PDWS. Aluminum, iron, and total organic halogens exceeded SRS Flag 2 criteria in both upgradient wells. Also, turbidity was elevated in upgradient well FAC 3.

Downgradient well FAC 6 was dry and could not be sampled. 


\section{Conclusions}

During second quarter 1994, gross alpha, aluminum, iron, manganese, and total organic halogens exceeded final PDWS or other Flag 2 criteria in FAC wells at the F-Area Acid/Caustic Basin. The SRS turbidity standard was exceeded in groundwater from well FAC 3 at 198 NTU. Samples from this well have consistently exceeded the turbidity standard in previous quarters.

During second quarter 1994, one or both of the upgradient wells, FAC 3 and 5 , contained elevated levels of aluminum, gross alpha, iron, total organic halogens, and turbidity. Downgradient well FAC 6 was dry and could not be sampled.

Because historical records indicate that no radionuclides were disposed of at this waste management unit (Heffner and Exploration Resources, 1991), the elevated level of radioactive constituents in the FAC wells is not considered to be due to seepage from the F-Area Acid/Caustic
Basin.

Water-table elevations from the FAC piezometers at the F-Area Acid/Caustic Basin indicate a northwest flow direction at a rate of approximately $1,30 \mathrm{ft} / \mathrm{yr}$. The groundwater flow depicted from the piezometers is a more realistic projection of flow conditions in the water table than the flow rate and direction obtained from monitoring well data. 


\section{References Cited}

Bergren, C. L., and C. B. Bennett, 1989. Assessment of SRS Groundwater Monitoring Wells
Impacted by Turbidity, WSRC-RP-89-891. Westinghouse Impacted by Turbidity, WSRC-RP-89-891. Westinghouse Savannah River Company, Aiken,

EPA (U.S. Environmental Protection Agency), 1986. RCRA Ground Water Monitoring Technical Enforcement Guidance Document, OSWER-9950.1. Washington, DC.

EPD/EMS, (Environmental Protection Department/Environmental Monitoring Section), 1992. Hydrogeologic Data Collection Procedures and Specifications: Sampling Groundwater Monitoring Wells, Manual 3Q5, Chapter 14, Revision 0. Environmental Protection Department, Environmental Monitoring Section, Savannah River Site, Aiken, SC.

EPD/EMS (Environmental Protection Department/Environmental Monitoring Section), 1994. Environmental Protection Department's Well Inventory (through the fourth quarter of 1993), ESH-EMS-930262, March 1994. Westinghouse Savannah River Company, Savannah River Site, Aiken, SC.

Gass, T. E., 1989. Monitoring Wells in Non-Aquifer Formations. Water Well Journal 43(2):27-29.

Geraghty \& Miller, Inc., 1990. Evaluation of Integrated Waste Facility Closure Capping on Ground-Water Flow and Solute Transport in General Separations Area, Savannah River Site: Flow Model and Particle-Tracking Analysis, Final Report. Prepared by Geraghty \& Miller Modeling Group for Westinghouse Savannah River Company, Waste Management Technology, Savannah River Site, Aiken, SC.

Heffner, J. D., and Exploration Resources, Inc., 1991. Technical Summary of Groundwater Quality Protection Program at the Savannah River Site (1952-1986), Volume I-Site Geohydrology and Waste Sites, DPSP-88-1002. Westinghouse Savannah River Company, Aiken, SC.

Killian, T. H., N. L. Kolb, P. Corbo, and I. W. Marine, 1987. F-Area Seepage Basins, DPST-85-704. Savannah River Laboratory, E. I. du Pont de Nemours \& Company, Aiken, SC.

WSRC (Westinghouse Savannah River Company), 1991. F-, H-, K-, and P-Area Acid/Caustic Basins Groundwater Quality Assessment Plan, WSRC-TR-91-178, Revision 1.0. Westinghouse Savannah River Company, Aiken, SC. 


\section{Errata}

In tables with four quarters of data, some values for earlier quarters may differ from values for those same quarters presented in earlier reports because some reanalyses may have been perSecond Quarter 1993 through Fourth Quarter 1993:

- No errata have been reported.

First Quarter 1994:

- Page 5, Groundwater Monitoring Data: The copper standard is the final PDWS established
by EPA. 


\section{Appendix A}

\section{Final Primary Drinking Water Standards}


THIS PAGE LEFT BLANK INTENTIIONALLY. 


\section{Final Primary Drinking Water Standards}

\begin{tabular}{|c|c|c|c|c|}
\hline Analyte & Unit & Level & Status & Source \\
\hline $\begin{array}{l}\text { Alachlor } \\
\text { Aldicarb }^{a}\end{array}$ & $\mu g / L$ & 2 & Final & EPA, 1993 \\
\hline Aldicarb sulfone ${ }^{a}$ & $\mu \mathrm{g} / \mathrm{L}$ & 3 & Final & EPA, 1993 \\
\hline $\begin{array}{l}\text { Aldicarb sulfone } e^{a} \\
\text { Aldicarb sulfoxide }\end{array}$ & $\mu \mathrm{g} / \mathrm{L}$ & 2 & Final & EPA, 1993 \\
\hline Antimony & $\mu g / L$ & 4 & Final & EPA, 1993 \\
\hline $\begin{array}{l}\text { Antimony } \\
\text { Antimony, dissolved }\end{array}$ & $\mu g / L$ & 6 & Final & EPA, 1993 \\
\hline $\begin{array}{l}\text { Antimony, dissolved } \\
\text { Antimony, total recoverable }\end{array}$ & $\mu \mathrm{g} / \mathrm{L}$ & 6 & Final & EPA, 1993 \\
\hline $\begin{array}{l}\text { Antimony, total recoverable } \\
\text { Arsenic }\end{array}$ & $\mu g / L$ & 6 & Final & EPA, 1993 \\
\hline $\begin{array}{l}\text { Arsenic } \\
\text { Arsenic, dissolved }\end{array}$ & $\mu \mathrm{g} / \mathrm{L}$ & 50 & Final & EPA, 1993 \\
\hline $\begin{array}{l}\text { Arsenic, dissolved } \\
\text { Arsenic, total recoverable }\end{array}$ & $\mu \mathrm{g} / \mathrm{L}$ & 50 & Final & EPA, 1993 \\
\hline Asbestos & $\mu g / L$ & 50 & Final & EPA, 1993 \\
\hline Atrazine & Fibers/L & $7,000,000$ & Final & EPA, 1993 \\
\hline $\begin{array}{l}\text { Atrazine } \\
\text { Barium }\end{array}$ & $\mu g / L$ & 3 & Final & EPA, 1993 \\
\hline Barium, dissolved & $\mu g / L$ & 2,000 & Final & EPA, 1993 \\
\hline Barium, total recoverable & $\mu \mathrm{g} / \mathrm{L}$ & 2,000 & Final & EPA, 1993 \\
\hline $\begin{array}{l}\text { Barium, total recoverable } \\
\text { Benzene }\end{array}$ & $\mu g / L$ & 2,000 & Fina! & EPA, 1993 \\
\hline $\begin{array}{l}\text { Benzene } \\
\text { Benzo[a]pyrene }\end{array}$ & $\mu \mathrm{g} / \mathrm{L}$ & 5 & Final & EPA, 1993 \\
\hline $\begin{array}{l}\text { Benzo[a]pyrene } \\
\text { Beryilium }\end{array}$ & $\mu \mathrm{g} / \mathrm{L}$ & 0.2 & Final & EPA, 1993 \\
\hline $\begin{array}{l}\text { Beryllium } \\
\text { Beryllium, dissolved }\end{array}$ & $\mu \mathrm{g} / \mathrm{L}$ & 4 & Final & EPA, 1993 \\
\hline & $\mu g / L$ & 4 & Final & EPA, 1993 \\
\hline $\begin{array}{l}\text { Beryllium, total recoverable } \\
\text { Bis(2-ethylhexyl) phthalate }\end{array}$ & $\mu g / L$ & 4 & Final & EPA, 1993 \\
\hline & $\mu \mathrm{g} / \mathrm{L}$ & 6 & Final & EPA, 1993 \\
\hline $\begin{array}{l}\text { Bromodichloromethane } \\
\text { Bromoform }\end{array}$ & $\mu g / L$ & 100 & Final & EPA, 1993 \\
\hline $\begin{array}{l}\text { Bromoform } \\
\text { 2-sec-Butyl-4,6-dinitrophenol }\end{array}$ & $\mu g / L$ & 100 & Final & EPA, 1993 \\
\hline $\begin{array}{l}\text { 2-sec-Butyl-4,6-dinitrophenol } \\
\text { Cadmium }\end{array}$ & $\mu g / L$ & 7 & Final & EPA, 1993 \\
\hline & $\mu \mathrm{g} / \mathrm{L}$ & 5 & Final & EPA, 1993 \\
\hline $\begin{array}{l}\text { Cadmium, dissolved } \\
\text { Cadmium, total recoverable }\end{array}$ & $\mu \mathrm{g} / \mathrm{L}$ & 5 & Final & EPA, 1993 \\
\hline $\begin{array}{l}\text { Cadmium, total recoverable } \\
\text { Carbofuran }\end{array}$ & $\mu g / L$ & 5 & Final & EPA, 1993 \\
\hline $\begin{array}{l}\text { Carbofuran } \\
\text { Carbon tetrachloride }\end{array}$ & $\mu g / L$ & 40 & Final & EPA, 1993 \\
\hline $\begin{array}{l}\text { Carbon tetrachloride } \\
\text { Chlordane }\end{array}$ & $\mu g / L$ & 5 & Final & EPA, 1993 \\
\hline $\begin{array}{l}\text { Chlordane } \\
\text { Chlorobenzene }\end{array}$ & $\mu g / L$ & 2 & Final & EPA, 1993 \\
\hline & $\mu g / L$ & 100 & Final & EPA, 1993 \\
\hline $\begin{array}{l}\text { Chloroethene (Vinyl chloride) } \\
\text { Chloroform }\end{array}$ & $\mu g / L$ & 2 & Final & EPA, 1993 \\
\hline $\begin{array}{l}\text { Chloroform } \\
\text { Chromium }\end{array}$ & $\mu g / L$ & 100 & Final & EPA, 1993 \\
\hline & $\mu g / L$ & 100 & Final & EPA, 1993 \\
\hline $\begin{array}{l}\text { Chromium, dissolved } \\
\text { Chromium, total recoverable }\end{array}$ & $\mu g / L$ & 100 & Final & EPA, 1993 \\
\hline $\begin{array}{l}\text { Chromium, total recoverable } \\
\text { Copper }\end{array}$ & $\mu g / L$ & 100 & Final & EPA, 1993 \\
\hline & $\mu g / L$ & 1,300 & Final & EPA, 1993 \\
\hline $\begin{array}{l}\text { Copper, dissolved } \\
\text { Copper, total recoverable }\end{array}$ & $\mu g / L$ & 1,300 & Final & EPA, 1993 \\
\hline $\begin{array}{l}\text { Copper, total recoverable } \\
\text { Cyanide }\end{array}$ & $\mu g / L$ & 1,300 & Final & EPA, 1993 \\
\hline $\begin{array}{l}\text { Cyanide } \\
\text { Dalapon }\end{array}$ & $\mu g / L$ & 200 & Final & EPA, 1993 \\
\hline & $\mu g / L$ & 200 & Final & EPA, 1993 \\
\hline $\begin{array}{l}\text { Dibromochloromethane } \\
\text { 1,2-Dibromo-3-chloropropane }\end{array}$ & $\mu g / L$ & 100 & Final & EPA, 1993 \\
\hline $\begin{array}{l}\text { 1,2-Dibromo-3-chloropropane } \\
\text { 1,2-Dibromoethane }\end{array}$ & $\mu g / L$ & 0.2 & Final & EPA, 1993 \\
\hline $\begin{array}{l}\text { 1,2-Dibromoethane } \\
\text { 1,2-Dichlorobenzene }\end{array}$ & $\mu g / L$ & 0.05 & Final & EPA, 1993 \\
\hline $\begin{array}{l}\text { 1,2-Dichlorobenzene } \\
\text { 1,4-Dichlorobenzene }\end{array}$ & $\mu g / L$ & 600 & Final & EPA, 1993 \\
\hline $\begin{array}{l}\text { 1,4-Dichlorobenzene } \\
\text { 1,2-Dichloroethane }\end{array}$ & $\mu g / L$ & 75 & Final & EPA, 1993 \\
\hline $\begin{array}{l}\text { 1,2-Dichloroethane } \\
\text { 1,1-Dichloroethylene }\end{array}$ & $\mu g / L$ & 5 & Final & EPA, 1993 \\
\hline $\begin{array}{l}\text { 1,1-Dichloroethylene } \\
\text { 1,2-Dichioroethylene }\end{array}$ & $\mu \mathrm{g} / \mathrm{L}$ & 7 & Final & EPA, 1993 \\
\hline $\begin{array}{l}\text { 1,2-Dichioroethylene } \\
\text { cis-1,2-Dichloroethylene }\end{array}$ & $\mu g / L$ & 50 & Final & EPA, 1993 \\
\hline $\begin{array}{l}\text { cis-1,2-Dichloroethylene } \\
\text { trans-1,2-Dichloroethylene }\end{array}$ & $\mu g / L$ & 70 & Final & EPA, 1993 \\
\hline & $\mu g / L$ & 100 & Final & EPA, 1993 \\
\hline $\begin{array}{l}\text { Dichloromethane (Methylene chloride) } \\
\text { 2,4-Dichlorophenoxyacetic acid }\end{array}$ & $\mu g / L$ & 5 & Final & EPA, 1993 \\
\hline 2,4-Dichlorophenoxyacetic acid & $\mu g / L$ & 70 & Final & EPA, 1993 \\
\hline
\end{tabular}




Analyte
1,2-Dichloropropane
Di(2-ethylhexyl) adipate
Diquat dibromide
Endothall
Endrin
Ethylbenzene
Fluoride
Glyphosate
Gross alpha
Heptachlor
Heptachlor epoxide
Hexachlorobenzene
Hexachlorocyclopentadiene
Lead
Lead, dissolved
Lead, total recoverable
Lindane
Mercury
Mercury, dissolved
Mercury, total recoverable
Methoxychlor
Nickel
Nickel, dissolved
Nickel, total recoverable
Nitrate as nitrogen
Nitrate-nitrite as nitrogen
Nitrite as nitrogen
Nonvolatile beta
Oxamyl
PCB 1016
PCB 1221
PCB 1232
PCB 1242
PCB 1248
PCB 1254
PCB 1260
PCB 1262
Pentachlorophenol
Piclorama
Selenium
Selenium, dissolved
Selenium, total recoverable
Simazine
Strontium-89/90
Strontium-90
Styrene
2,3,7,8-TCDD
Tetrachloroethylene
Thallium
Thallium, dissolved
Thallium, total recoverable
Toluene
Toxaphene
2,4,5-TP (Silvex)
1,2,4-Trichlorobenzene

\begin{tabular}{|c|c|c|c|}
\hline Unit & Level & $\underline{\text { Status }}$ & Source \\
\hline$\mu \mathrm{g} / \mathrm{L}$ & 5 & Final & EPA, 1993 \\
\hline$\mu g / L$ & 400 & Final & EPA, 1993 \\
\hline$\mu g / L$ & 20 & Final & EPA, 1993 \\
\hline$\mu g / L$ & 100 & Final & EPA, 1993 \\
\hline$\mu g / L$ & 2 & Final & EPA, 1993 \\
\hline$\mu g / L$ & 700 & Final & EPA, 1993 \\
\hline$\mu \mathrm{g} / \mathrm{L}$ & 4,000 & Final & EPA, 1993 \\
\hline$\mu g / L$ & 700 & Final & EPA, 1993 \\
\hline $\mathrm{pCi} / \mathrm{L}$ & $1.5 E+01$ & Final & EPA, 1993 \\
\hline$\mu \mathrm{g} / \mathrm{L}$ & 0.4 & Final & EPA, 1993 \\
\hline$\mu g / L$ & 0.2 & Final & EPA, 1993 \\
\hline$\mu g / L$ & 1 & Final & EPA, 1993 \\
\hline$\mu g / L$ & 50 & Final & EPA, 1993 \\
\hline$\mu g / L$ & 50 & Final & SCDHEC, 1981 \\
\hline$\mu \mathrm{g} / \mathrm{L}$ & 50 & Final & SCDHEC, 1981 \\
\hline$\mu g / L$ & 50 & Final & SCDHEC, 1981 \\
\hline$\mu g / L$ & 0.2 & Final & EPA, 1993 \\
\hline$\mu \mathrm{g} / \mathrm{L}$ & 2 & Final & EPA, 1993 \\
\hline$\mu \mathrm{g} / \mathrm{L}$ & 2 & Final & EPA, 1993 \\
\hline$\mu g / L$ & 2 & Final & EPA, 1993 \\
\hline$\mu g / L$ & 40 & Final & EPA, 1993 \\
\hline$\mu g / L$ & 100 & Final & EPA, 1993 \\
\hline$\mu g / L$ & 100 & Final & EPA, 1993 \\
\hline$\mu \mathrm{g} / \mathrm{L}$ & 100 & Final & EPA, 1993 \\
\hline$\mu g / L$ & 10,000 & Final & EPA, 1993 \\
\hline$\mu g / L$ & 10,000 & Final & EPA, 1993 \\
\hline$\mu \mathrm{g} / \mathrm{L}$ & 1,000 & Final & EPA, 1993 \\
\hline $\mathrm{pCi} / \mathrm{L}$ & $5 \mathrm{E}+01$ & Interim Final & EPA, 1977 \\
\hline$\mu g / L$ & 200 & Final & EPA, 1993 \\
\hline$\mu g / L$ & 0.5 & Final & EPA, 1993 \\
\hline$\mu \mathrm{g} / \mathrm{h}$ & 0.5 & Final & EPA, 1993 \\
\hline$\mu \mathrm{g} / \mathrm{L}$ & 0.5 & Final & EPA, 1993 \\
\hline$\mu g / L$ & 0.5 & Final & EPA, 1993 \\
\hline$\mu \mathrm{g} / \mathrm{L}$ & 0.5 & Final & EPA, 1993 \\
\hline$\mu g / L$ & 0.5 & Final & EPA, 1993 \\
\hline$\mu g / L$ & 0.5 & Final & EPA, 1993 \\
\hline$\mu g / L$ & 0.5 & Final & EPA, 1993 \\
\hline$\mu g / L$ & 1 & Final & EPA, 1993 \\
\hline$\mu \mathrm{g} / \mathrm{L}$ & 500 & Final & EPA, 1993 \\
\hline$\mu g / h$ & 50 & Final & EPA, 1993 \\
\hline$\mu \mathrm{g} / \mathrm{L}$ & 50 & Final & EPA, 1993 \\
\hline$\mu g / L$ & 50 & Final & EPA, 1993 \\
\hline$\mu g / L$ & 4 & Final & EPA, 1993 \\
\hline $\mathrm{pCi} / \mathrm{L}$ & $8 E+00$ & Final & EPA, 1993 \\
\hline pCi/L & $8 E+00$ & Final & EPA, 1993 \\
\hline$\mu g / L$ & 100 & Final & EPA, 1993 \\
\hline$\mu g / L$ & 0.00003 & Final & EPA, 1993 \\
\hline$\mu \mathrm{g} / \mathrm{L}$ & 5 & Final & EPA, 1993 \\
\hline$\mu g / L$ & 2 & Final & EPA, 1993 \\
\hline$\mu g / L$ & 2 & Final & EPA, 1993 \\
\hline$\mu g / L$ & & Final & EPA, 1993 \\
\hline$\mu g / L$ & 1,000 & Final & EPA, 1993 \\
\hline$\mu g / L$ & 3 & Final & EPA, 1993 \\
\hline$\mu g / L$ & 50 & Final & EPA, 1993 \\
\hline$\mu g / L$ & 70 & Final & EPA, 1993 \\
\hline
\end{tabular}


Analyte

1,1,1-Trichloroethane

1,1,2-Trichloroethane

Trichloroethylene

Tritium

Xylenes

$\begin{array}{llll}\text { Unit } & \text { Level } & \text { Status } & \text { Source } \\ \mu \mathrm{g} / \mathrm{L} & 200 & \text { Final } & \text { EPA, 1993 } \\ \mu \mathrm{g} / \mathrm{L} & 5 & \text { Final } & \text { EPA, 1993 } \\ \mu \mathrm{g} / \mathrm{L} & 5 & \text { Final } & \text { EPA, 1993 } \\ \mathrm{pCi} / \mathrm{mL} & 2 \mathrm{E}+01 & \text { Final } & \text { EPA, 1993 } \\ \mu \mathrm{g} / \mathrm{L} & 10,000 & \text { Final } & \text { EPA, 1993 }\end{array}$

Note: Final PDWS were assigned to alachlor, aldicarb, aldicarb sulfone, aldicarb sulfoxide, atrazine, carbofuran, dalapon, di(2-ethylhexyl) adipate, diquat dibromide, endothall, glyphosate, oxamyl, picloram, and simazine in the SRS Groundwater Monitoring Program for the first time beginning first quarter 1994.

a At present, EMS does not perform this analysis because the constituent is not in the current contract.

b The standard given is for gross alpha including radium-226 but excluding radon and uranium.

c For double radionuctide analyses where each separate radionuclide has its own standard, the stringent standard is used.

\section{References}

EPA (U.S. Environmental Protection Agency), 1977. National Interim Primary Drinking Water Regulations, EPA-570/9-76-003. Washington, DC.

EPA (U.S. Environmental Protection Agency), 1993. National Primary Drinking Water Regulations, Code of Federal Regulations, Section 40, Part 141, pp. 592-732. Washington, DC

SCDHEC (South Carolina Department of Health and Environmental Control), 1981. State Primary Drinking Water Regulations, R.61-58.5. Columbia, SC. 
WSRC-TR-94-0347

Unclassified

THIS PAGE LEFT BLANK INTENTIONALLY. 


\section{Appendix B}

Flagging Criteria 
THIS PAGE LEFT BLANK INTENTIONALLY. 


\section{Flagging Criteria}

The Savannah River Site Environmental Protection Department/Environmental Monitoring Section (EPD/EMS) flagging criteria are as follows:

- Flag 2 criteria for constituents equal the Safe Drinking Water Act (SDWA) final Primary Drinking Water Standard (PDWS), the SDWA proposed PDWS, or the SDWA Secondary Drinking Water Standard (SDWS). If a constituent does not have a drinking water standard, the Flag 2 criterion equals 10 times the method detection limit (MDL) calculated as the 90th percentile detection limit obtained recently by one of the primary analytical laboratories.

- Flag 1 criteria for constituents equal one-half of the final PDWS, one-half the proposed PDWS, or one-half the SDWS. If a constituent does not have a drinking water standard, the Flag 1 criterion equals 5 times the MDL calculated as the 90th percentile detection limit obtained recently by one of the primary analytical laboratories.

- Flag 0 criteria are assigned to constituent levels below Flag 1 criteria, constituent levels below the sample detection limits, or constituents having no flagging criteria.

The following parameters are exceptions to the flagging rules:

- EPD/EMS sets flagging criteria for specific conductance and $\mathrm{pH}$. No flags are set for alkalinity, calcium, carbonate, magnesium, potassium, silica, sodium, total dissolved solids, total phosphates (as P), and total phosphorus. Analyses for these parameters are conducted as part of the biennial comprehensive analyses or by special request.

- Aesthetic parameters such as color, corrosivity, Eh, odor, surfactants, and turbidity are not assigned flagging criteria but are analyzed by special request.

- Common laboratory contaminants and cleaners such as dichloromethane (methylene chloride), ketones, phthalates, and toluene are not assigned flagging criteria unless they have primary drinking water standards. These constituents are analyzed by special request.

\begin{tabular}{|c|c|c|c|c|}
\hline Analyte & Unit & Flag 1 & Flag 2 & $\underline{\text { Source }}^{\mathrm{a}}$ \\
\hline Acenaphthene & $\mu g / L$ & 50 & 100 & EPA Method 8270 \\
\hline Acenaphthylene & $\mu g / L$ & 50 & 100 & EPA Method 8270 \\
\hline Acetone & $\mu g / L$ & 500 & 1,000 & EPA Method 8240 \\
\hline Acetonitrile (Methyl cyanide) & $\mu g / L$ & 500 & 1,000 & EPA Method 8240 \\
\hline Acetophenone & $\mu g / L$ & 50 & 100 & EPA Method 8270 \\
\hline 2-Acetylaminofluorene & $\mu g / L$ & 50 & 100 & EPA Method 8270 \\
\hline Acrolein & $\mu g / L$ & 100 & 200 & EPA Method 8240 \\
\hline Acrylonitrile & $\mu g h$ & 100 & 200 & EPA Method 8240 \\
\hline Actinium-228 & $\mathrm{pCi} / \mathrm{L}$ & $1.64 E+03$ & $3.27 E+03$ & Proposed PDWS (EPA, 1991) \\
\hline Alachlor & $\mu \mathrm{g} / L$ & 1 & 2 & Final PDWS (EPA, 1993a) \\
\hline Aldicarb ${ }^{b}$ & $\mu g / L$ & 1.5 & 3 & Final PDWS (EPA, 1993a) \\
\hline Aldicarb sulfone $e^{b}$ & $\mu \mathrm{g} / \mathrm{L}$ & 1 & 2 & Final PDWS (EPA, 1993a) \\
\hline Aldicarb sulfoxide ${ }^{b}$ & $\mu g / L$ & 2 & 4 & Final PDWS (EPA, 1993a) \\
\hline Aldrin & $\mu g / L$ & 0.25 & 0.5 & EPA Method 8080 \\
\hline Alkalinity (as $\mathrm{CaCO}_{3}$ ) & & No flag & No flag & Set by EPD/EMS \\
\hline Allyl chloride & $\mu g / L$ & 250 & 500 & EPA Method 8240 \\
\hline Aluminum & $\mu g / L$ & 25 & 50 & SDWS (EPA, 1993b) \\
\hline Aluminum, dissolved & $\mu g / L$ & 25 & 50 & SDWS (EPA, 1993b) \\
\hline Aluminum, total recoverable & $\mu \mathrm{g} / \mathrm{L}$ & 25 & 50 & SDWS (EPA, 1993b) \\
\hline
\end{tabular}




Analyte
Americium-241
Americium-243
4-Aminobiphenyl
Ammonia
Ammonia nitrogen
Aniline
Anthracene
Antimony
Antimony, dissolved
Antimony, total recoverable
Antimony-125
Aramite
Arsenic
Arsenic, dissolved
Arsenic, total recoverable
Asbestos
Atrazine
Azobenzene
Barium
Barium, dissolved
Barium, total recoverable
Barium-140c
Benzene
alpha-Benzene hexachloride
beta-Benzene hexachloride
delta-Benzene hexachloride
Benzidine
Benzo[a]anthracene
Benzo[b]fluoranthene
Benzo[k]fluoranthene
Benzoic acid
Benzo[g, h,]perylene
Benzo[a]pyrene
1,4-Benzoquinone
Benzyl alcohol
Beryllium
Beryllium, dissolved
Beryllium, total recoverable
Beryllium-7
Bis(2-chloroethoxy) methane
Bis(2-chloroethyl) ether
Bis(2-chloroisopropyl) ether
Bis(chloromethyl) ether
Bis(2-ethylhexyl) phthalate
Bismuth-214
Boron
Boron, dissolved
Boron, total recoverable
Bromide
Bromodichloromethane
Bromoform
Bromomethane (Methyl bromide)
4-Bromophenyl phenyl ether
2-sec-Butyl-4,6-dinitrophenol
Butylbenzyl phthalate

\begin{tabular}{|c|c|c|}
\hline Unit & Flag 1 & Flag 2 \\
\hline $\mathrm{pCi} / \mathrm{L}$ & $3.17 E+00$ & $6.34 E+00$ \\
\hline $\mathrm{pCi} / \mathrm{L}$ & $3.19 E+00$ & $6.37 E+00$ \\
\hline$\mu g / L$ & 50 & 100 \\
\hline$\mu g / L$ & 500 & 1,000 \\
\hline$\mu g / L$ & 500 & 1,000 \\
\hline$\mu g / L$ & 50 & 100 \\
\hline$\mu g / L$ & 50 & 100 \\
\hline$\mu g / L$ & 3 & 6 \\
\hline$\mu g / L$ & 3 & 6 \\
\hline$\mu g / L$ & 3 & 6 \\
\hline $\mathrm{pCi} / \mathrm{L}$ & $1.5 E+02$ & $3 E+02$ \\
\hline$\mu g / L$ & 50 & 100 \\
\hline$\mu g / L$ & 25 & 50 \\
\hline$\mu g / L$ & 25 & 50 \\
\hline$\mu g / L$ & 25 & 50 \\
\hline Fibers & $-3,500,000$ & $7,000,000$ \\
\hline$\mu g / L$ & 1.5 & 3 \\
\hline$\mu g / L$ & 50 & 100 \\
\hline$\mu g / h$ & 1,000 & 2,000 \\
\hline$\mu g / L$ & 1,000 & 2,000 \\
\hline$\mu g / L$ & 1,000 & 2,000 \\
\hline $\mathrm{pCi} / \mathrm{L}$ & $4.5 \mathrm{E}+01$ & $9 \mathrm{E}+01$ \\
\hline$\mu g / L$ & 2.5 & 5 \\
\hline$\mu g / L$ & 0.25 & 0.5 \\
\hline$\mu \mathrm{g} / \mathrm{L}$ & 0.25 & 0.5 \\
\hline$\mu g / L$ & 0.25 & 0.5 \\
\hline$\mu g / L$ & 250 & 500 \\
\hline$\mu g / L$ & 0.05 & 0.1 \\
\hline$\mu g / L$ & 0.1 & 0.2 \\
\hline$\mu g / L$ & 0.1 & 0.2 \\
\hline$\mu g / L$ & 250 & 500 \\
\hline$\mu g / L$ & 50 & 100 \\
\hline$\mu \mathrm{g} / \mathrm{L}$ & 0.1 & 0.2 \\
\hline$\mu g / L$ & 50 & 100 \\
\hline$\mu g / L$ & 50 & 100 \\
\hline$\mu g / L$ & 2 & $\begin{array}{l}4 \\
4\end{array}$ \\
\hline $\begin{array}{l}\mu g / L \\
\mu g / L\end{array}$ & 2 & $\begin{array}{l}4 \\
4\end{array}$ \\
\hline $\begin{array}{l}\mu g / L \\
\mathrm{pCi} / \mathrm{L}\end{array}$ & $\begin{array}{l}2 \\
3 E+03\end{array}$ & $\begin{array}{l}4 \\
6 E+03\end{array}$ \\
\hline $\begin{array}{l}\mathrm{pCl} / \mathrm{L} \\
\mu \mathrm{g} / \mathrm{L}\end{array}$ & $\begin{array}{l}3 E+03 \\
50\end{array}$ & 100 \\
\hline$\mu g / L$ & 50 & 100 \\
\hline$\mu g / L$ & 50 & 100 \\
\hline$\mu \mathrm{g} / \mathrm{L}$ & 50 & 100 \\
\hline$\mu g / L$ & 3 & 6 \\
\hline $\mathrm{pCi} / \mathrm{L}$ & $9.4 \mathrm{E}+03$ & $1.89 E+04$ \\
\hline$\mu g / \Omega$ & 150 & 300 \\
\hline$\mu g / L$ & 150 & 300 \\
\hline$\mu g / h$ & 150 & 300 \\
\hline$\mu g / L$ & 5,000 & 10,000 \\
\hline$\mu g / L$ & 50 & 100 \\
\hline$\mu g / h$ & 50 & 100 \\
\hline$\mu g / L$ & 5 & 10 \\
\hline$\mu g / h$ & 50 & 100 \\
\hline$\mu g / L$ & 3.5 & 7 \\
\hline & No flag & No flag \\
\hline
\end{tabular}

$\underline{\text { Source }}^{a}$

Proposed PDWS (EPA, 1991)

Proposed PDWS (EPA, 1991)

EPA Method 8270

APHA Method 417B

EPA Method 350.1

EPA Method 8270

EPA Method 8270

Final PDWS (EPA, 1993a)

Final PDWS (EPA, 1993a)

Final PDWS (EPA, 1993a)

Interim Final PDWS (EPA, 1977)

EPA Method 8270

Final PDWS (EPA, 1993a)

Final PDWS (EPA, 1993a)

Final PDWS (EPA, 1993a)

Final PDWS (EPA, 1993a)

Final PDWS (EPA, 1993a)

EPA Method 625

Final PDWS (EPA, 1993a)

Final PDWS (EPA, 1993a)

Final PDWS (EPA, 1993a)

Interim Final PDWS (EPA, 1977)

Final PDWS (EPA, 1993a)

EPA Method 8080

EPA Method 8080

EPA Method 8080

EPA Method 8270

Proposed PDWS (EPA, 1990)

Proposed PDWS (EPA, 1990)

Proposed PDWS (EPA, 1990)

EPA Method 8270

EPA Method 8270

Final PDWS (EPA, 1993a)

EPA Method 8270

EPA Method 8270

Final PDWS (EPA, 1993a)

Final PDWS (EPA, 1993a)

Final PDWS (EPA, 1993a)

Interim Final PDWS (EPA, 1977)

EPA Method 8270

EPA Method 8270

EPA Method 8270

EPA Method 8270

Final PDWS (EPA, 1993a)

Proposed PDWS (EPA, 1991)

EPA Method 6010

EPA Method 6010

EPA Method 6010

EPA Method 300.0

Final PDWS (EPA, 1993a)

Final PDWS (EPA, 1993a)

EPA Method 8240

EPA Method 8270

Final PDWS (EPA, 1993a)

Set by EPD/EMS 


Analyte
Cadmium
Cadmium, dissolved
Cadmium, total recoverable
Calcium
Calcium, dissolved
Calcium, total recoverable
Carbon disulfide
Carbofuran
Carbon tetrachloride
Carbon-14
Carbonate
Cerium-141
Cerium-144
Cesium-134
Cesium-137
Chlordane
Chloride
4-Chloroaniline
Chlorobenzene
Chlorobenzilate
Chloroethane
Chloroethene (Vinyl chloride)
Chloroethyl vinyl ether
2-Chloroethyl vinyl ether
Chloroform
4-Chloro-m-cresol
Chloromethane (Methyl chloride)
2-Chloronaphthalene
2-Chlorophenol
4-Chlorophenyl phenyl ether
Chloroprene
Chromium
Chromium, dissolved
Chromium, total recoverable
Chromium-51
Chrysene
Cobalt
Cobalt, dissolved
Cobalt, total recoverable
Cobalt-57
Cobalt-58
Cobalt-60
Color
Copper
Copper, dissolved
Copper, total recoverable
Corrosivity
m-Cresol (3-Methylphenol)
o-Cresol (2-Methylphenol)
p-Cresol (4-Methylphenol)
Curium-242
Curium-243
Curium-243/244e
Curium-244
Curium-245/246

\begin{tabular}{|c|c|c|}
\hline Unit & Flag 1 & Flag 2 \\
\hline$\mu \mathrm{g} / \mathrm{L}$ & 2.5 & 5 \\
\hline$\mu \mathrm{g} / \mathrm{L}$ & 2.5 & 5 \\
\hline$\mu \mathrm{g} / \mathrm{L}$ & 2.5 & 5 \\
\hline & $\begin{array}{l}\text { No flag } \\
\text { No flag }\end{array}$ & $\begin{array}{l}\text { No flag } \\
\text { No flag }\end{array}$ \\
\hline$\mu \mathrm{g} / \mathrm{L}$ & $\begin{array}{l}\text { No flag } \\
5\end{array}$ & No flag \\
\hline$\mu \mathrm{g} / \mathrm{L}$ & $\begin{array}{l}5 \\
20\end{array}$ & $\begin{array}{l}10 \\
40\end{array}$ \\
\hline$\mu \mathrm{g} / \mathrm{L}$ & 2.5 & $\begin{array}{l}40 \\
5\end{array}$ \\
\hline $\mathrm{pCi} / \mathrm{L}$ & $1 \mathrm{E}+03$ & $\begin{array}{l}5 \\
2 \mathrm{E} \\
+03\end{array}$ \\
\hline & No flag & No flag \\
\hline $\mathrm{pCi} / \mathrm{L}$ & $1.5 E+02$ & $3 E+02$ \\
\hline $\mathrm{pCi} / \mathrm{L}$ & $1.31 E+02$ & $2.61 E+02$ \\
\hline $\mathrm{pCi} / \mathrm{L}$ & 4.07E+01 & $8.13 E+01$ \\
\hline $\mathrm{pCi} / \mathrm{L}$ & $1 \mathrm{E}+02$ & $2 E+02$ \\
\hline$\mu \mathrm{g} / \mathrm{L}$ & 1 & 2 \\
\hline$\mu \mathrm{g} / \mathrm{L}$ & 125,000 & 250,000 \\
\hline$\mu g / L$ & 50 & 100 \\
\hline$\mu g / L$ & 50 & 100 \\
\hline$\mu g / L$ & 50 & 100 \\
\hline$\mu \mathrm{g} / \mathrm{L}$ & 5 & 10 \\
\hline$\mu g / L$ & 1 & 2 \\
\hline$\mu g / L$ & 5 & 10 \\
\hline$\mu \mathrm{g} / \mathrm{L}$ & 5 & 10 \\
\hline$\mu g / L$ & 50 & 100 \\
\hline$\mu g / L$ & 50 & 100 \\
\hline$\mu g / L$ & 5 & 10 \\
\hline$\mu g / L$ & 50 & 100 \\
\hline$\mu \mathrm{g} / \mathrm{L}$ & 50 & 100 \\
\hline$\mu g / L$ & 50 & 100 \\
\hline$\mu \mathrm{g} / \mathrm{L}$ & 1,000 & 2,000 \\
\hline$\mu g / L$ & 50 & 100 \\
\hline$\mu g / L$ & 50 & 100 \\
\hline$\mu g / L$ & 50 & 100 \\
\hline $\mathrm{pCi} / \mathrm{L}$ & $3 E+03$ & $6 \mathrm{E}+03$ \\
\hline$\mu g / L$ & 0.1 & 0.2 \\
\hline$\mu \mathrm{g} / \mathrm{L}$ & 20 & 40 \\
\hline$\mu g / L$ & 20 & 40 \\
\hline$\mu g / L$ & 20 & 40 \\
\hline $\mathrm{pC} \mathrm{i} / \mathrm{L}$ & $5 E+02$ & $1 E+03$ \\
\hline $\mathrm{pCi} / \mathrm{L}$ & $4.5 E+03$ & $9 E+03$ \\
\hline $\mathrm{pCi} / \mathrm{L}$ & $\begin{array}{l}5 E+01 \\
\text { No flag }\end{array}$ & $1 \mathrm{E}+02$ \\
\hline$\mu g / L$ & $\begin{array}{l}\text { No flag } \\
500\end{array}$ & $\begin{array}{l}\text { No flag } \\
1,000\end{array}$ \\
\hline$\mu g / L$ & 500 & 1,000 \\
\hline$\mu g / L$ & 500 & 1,000 \\
\hline & No flag & No flag \\
\hline$\mu \mathrm{g} / \mathrm{L}$ & 50 & 100 \\
\hline$\mu g / L$ & 50 & 100 \\
\hline$y g / L$ & 50 & 100 \\
\hline $\mathrm{oCin}$ & $6.65 \mathrm{E}+01$ & $1.33 E+02$ \\
\hline $\mathrm{OCi} / \mathrm{L}$ & $4.15 \mathrm{E}+00$ & $8.3 E+00$ \\
\hline $\mathrm{Cl} / \mathrm{L}$ & $4.15 E+00$ & $8.3 E+00$ \\
\hline $\mathrm{Ci} / \mathrm{L}$ & $4.92 E+00$ & $9.84 E+00$ \\
\hline $\mathrm{Ci} / \mathrm{L}$ & $3.12 \mathrm{E}+00$ & $6.23 E+00$ \\
\hline
\end{tabular}

\section{Source}

Final PDWS (EPA, 1993a)

Final PDWS (EPA, 1993a)

Final PDWS (EPA, 1993a)

Set by EPD/EMS

Set by EPD/EMS

Set by EPD/EMS

EPA Method 8240

Final PDWS (EPA, 1993a)

Final PDWS (EPA, 1993a)

Interim Final PDWS (EPA, 1977)

Set by EPD/EMS

Interim Final PDWS (EPA, 1977)

Proposed PDWS (EPA, 1991)

Proposed PDWS (EPA, 1991)

Interim Final PDWS (EPA, 1977)

Final PDWS (EPA, 1993a)

SDWS (EPA, 1993b)

EPA Method 8270

Final PDWS (EPA, 1993a)

EPA Method 8270

EPA Method 8240

Final PDWS (EPA, 1993a)

EPA Method 8240

EPA Method 8240

Final PDWS (EPA, 1993a)

EPA Method 8270

EPA Method 8240

EPA Method 8240

EPA Method 8270

EPA Method 8270

EPA Method 8240

Final PDWS (EPA, 1993a)

Final PDWS (EPA, 1993a)

Final PDWS (EPA, 1993a)

Interim Final PDWS (EPA, 1977)

Proposed PDWS (EPA, 1990)

EPA Method 6010

EPA Method 6010

EPA Method 6010

Interim Final PDWS (EPA, 1977)

Interim Final PDWS (EPA, 1977)

Interim Final PDWS (EPA, 1977)

Set by EPD/EMS

Final PDWS (SCDHEC, 1981)

Final PDWS (SCDHEC, 1981)

Final PDWS (SCDHEC, 1981)

Set by EPD/EMS

EPA Method 8270

EPA Method 8270

EPA Method 8270

Proposed PDWS (EPA, 1991)

Proposed PDWS (EPA, 1991)

Proposed PDWS (EPA, 1991)

Proposed PDWS (EPA, 1991)

Proposed PDWS (EPA, 1991) 


Analyte
Curium-246
Cyanide
Dalapon
p,p'-DDD
p,p'-DDE
p,p'-DDT
Di-n-butyl phthalate
Di-n-octyl phthalate
Diallate
Dibenzla,h]anthracene
Dibenzofuran
Dibromochloromethane
1,2-Dibromo-3-chloropropane
1,2-Dibromoethane
Dibromomethane
(Methylene bromide)
1,2-Dichlorobenzene
1,3-Dichlorobenzene
1,4-Dichlorobenzene
3,3'-Dichlorobenzidine
trans-1,4-Dichloro-2-butene
Dichlorodiffuoromethane
1,1-Dichloroethane
1,2-Dichloroethane
1,1-Dichloroethylene
1,2-Dichloroethylene
cis-1,2-Dichloroethylene
trans-1,2-Dichloroethylene
Dichloromethane
(Methylene chloride)
2,4-Dichlorophenol
2,6-Dichlorophenol
2,4-Dichlorophenoxyacetic acid
1,2-Dichloropropane
cis-1,3-Dichloropropene
trans-1,3-Dichloropropene
Di(2-ethylhexyl) adipate
Dieldrin
Diethyl phthalate
Dimethoate
p-Dimethylaminoazobenzene
p-(Dimethylamino)ethylbenzene
7,12-Dimethylbenz[a]anthracene
3,3'-Dimethylbenzidine
a,a-Dimethylphenethylamine
2,4-Dimethyl phenol
Dimethyl phthalate
1,3-Dinitrobenzene
2,4-Dinitrophenol
2,4-Dinitrotoluene
2,6-Dinitrotoluene
Diquat dibromide
1,4-Dioxane
Diphenylamine
1,2-Diphenylhydrazine

\begin{tabular}{|c|c|c|c|}
\hline Unit & Flag 1 & Flag 2 & Source \\
\hline $\mathrm{PCi} / \mathrm{L}$ & $3.14 E+\infty 0$ & $6.27 \mathrm{E}+00$ & Proposed PDWS (EPA, 1991) \\
\hline$\mu g / L$ & 100 & 200 & Final PDWS (EPA, 1993a) \\
\hline$\mu g / L$ & 100 & 200 & Final PDWS (EPA, 1993a) \\
\hline$\mu g / L$ & 0.5 & 1 & EPA Method 8080 \\
\hline$\mu g / L$ & 0.5 & 1 & EPA Method 8080 \\
\hline$\mu \mathrm{g} / \mathrm{L}$ & 0.5 & & EPA Method 8080 \\
\hline & $\begin{array}{l}\text { No flag } \\
\text { No flag }\end{array}$ & No flag & $\begin{array}{l}\text { Set by EPD/EMS } \\
\text { Set by EPD/EMS }\end{array}$ \\
\hline$\mu \mathrm{g} / \mathrm{L}$ & & $\begin{array}{l}\text { No flag } \\
100\end{array}$ & $\begin{array}{l}\text { Set by EPD/EMS } \\
\text { EPA Method } 8270\end{array}$ \\
\hline$\mu \mathrm{g} / \mathrm{L}$ & 0.15 & 0.3 & Proposed PDWS (EPA, 1990) \\
\hline$\mu g / L$ & 50 & 100 & EPA Method 8270 \\
\hline$\mu \mathrm{g} / \mathrm{L}$ & 50 & 100 & Final PDWS (EPA, 1993a) \\
\hline$\mu g / L$ & 0.1 & 0.2 & Final PDWS (EPA, 1993a) \\
\hline $\begin{array}{l}\mu g / L \\
\mu g / L\end{array}$ & $\begin{array}{l}0.025 \\
5\end{array}$ & $\begin{array}{l}0.05 \\
10\end{array}$ & $\begin{array}{l}\text { Final PDWS (EPA, 1993a) } \\
\text { EPA Method } 8240\end{array}$ \\
\hline & & & \\
\hline$\mu g / L$ & $\begin{array}{l}300 \\
50\end{array}$ & 600 & Final PDWS (EPA, 1993a) \\
\hline$\mu \mathrm{g} / \mathrm{L}$ & $\begin{array}{l}50 \\
37.5\end{array}$ & 100 & EPA Method 8270 \\
\hline$\mu g / L$ & $\begin{array}{l}37.5 \\
50\end{array}$ & $\begin{array}{l}75 \\
100\end{array}$ & Final PDWS (EPA, 1993a) \\
\hline$\mu g / h$ & 150 & $\begin{array}{l}100 \\
300\end{array}$ & EPA Method 8270 \\
\hline$\mu g / L$ & 5 & $\begin{array}{l}300 \\
10\end{array}$ & EPA Method 8240 \\
\hline$\mu g / L$ & 5 & $\begin{array}{l}10 \\
10\end{array}$ & EPA Method 8240 \\
\hline$\mu g / L$ & 2.5 & $\begin{array}{l}10 \\
5\end{array}$ & EPA Method 8240 \\
\hline$\mu g / L$ & 3.5 & $\begin{array}{l}5 \\
7\end{array}$ & Final PDWS (EPA, 1993a) \\
\hline$\mu \mathrm{g} / \mathrm{L}$ & 25 & $\begin{array}{l}7 \\
50\end{array}$ & Final PDWS (EPA, 1993a) \\
\hline$\mu g / L$ & 35 & $\begin{array}{l}50 \\
70\end{array}$ & Final PDWS (EPA, 1993a) \\
\hline$\mu g / L$ & 50 & $\begin{array}{l}70 \\
100\end{array}$ & Final PDWS (EPA, 1993a) \\
\hline$\mu \mathrm{g} / \mathrm{L}$ & 2.5 & $\begin{array}{l}100 \\
5\end{array}$ & $\begin{array}{l}\text { Final PDWS (EPA, 1993a) } \\
\text { Final PDWS (EPA, 1993a) }\end{array}$ \\
\hline & & & Thal PUVV (EPA, 1993a) \\
\hline$\mu g / L$ & 50 & 100 & EPA Method 8270 \\
\hline$\mu g / L$ & 50 & 100 & EPA Method 8270 \\
\hline$\mu \mathrm{g} / \mathrm{L}$ & 35 & 70 & Final PDWS (EPA, 1993a) \\
\hline$\mu g / L$ & 2.5 & 5 & Final PDWS (EPA, 1993a) \\
\hline$\mu \mathrm{g} / \mathrm{L}$ & 5 & 10 & EPA Method 8240 \\
\hline$\mu g / L$ & 5 & 10 & EPA Method 8240 \\
\hline$\mu g / L$ & 200 & 400 & Final PDWS (EPA, 1993a) \\
\hline$\mu g / L$ & 2.5 & & EPA Method 8080 \\
\hline & No flag & No flag & Set by EPD/EMS \\
\hline$\mu g / L$ & 50 & 100 & $\begin{array}{l}\text { EPA Method } 8270 \\
\text { EPA Method } 8270\end{array}$ \\
\hline$\mu g / L$ & 50 & 100 & $\begin{array}{l}\text { EPA Method } 8270 \\
\text { EPA Method } 8270\end{array}$ \\
\hline$\mu g / L$ & 50 & $\begin{array}{l}100 \\
100\end{array}$ & $\begin{array}{l}\text { EPA Method } 8270 \\
\text { EPA Method } 8270\end{array}$ \\
\hline$\mu g / L$ & $\begin{array}{l}50 \\
50\end{array}$ & $\begin{array}{l}100 \\
100\end{array}$ & $\begin{array}{l}\text { EPA Method } 8270 \\
\text { EPA Method } 8270\end{array}$ \\
\hline $\begin{array}{l}\mu g / L \\
\mu g / L\end{array}$ & $\begin{array}{l}50 \\
50\end{array}$ & $\begin{array}{l}100 \\
100\end{array}$ & EPA Method 8270 \\
\hline$\mu \mathrm{g} / \mathrm{L}$ & & 100 & EPA Method 8270 \\
\hline & No flag & No flag & Set by EPD/EMS \\
\hline$\mu \mathrm{g} / \mathrm{L}$ & & 100 & EPA Method 8270 \\
\hline$\mu g / L$ & 250 & $\begin{array}{l}500 \\
100\end{array}$ & $\begin{array}{l}\text { EPA Method } 8270 \\
\text { EPA Method } 8270\end{array}$ \\
\hline$\mu g / L$ & $\begin{array}{l}50 \\
50\end{array}$ & $\begin{array}{l}100 \\
100\end{array}$ & $\begin{array}{l}\text { EPA Method } 8270 \\
\text { EPA Method } 8270\end{array}$ \\
\hline$u g / h$ & 10 & $\begin{array}{l}100 \\
20\end{array}$ & Final PDWS (EPA, 1993a) \\
\hline$u g / L$ & 50 & 100 & EPA Method 8270 \\
\hline$\mu g / L$ & 50 & 100 & EPA Method 8270 \\
\hline $\mathrm{ug} / \mathrm{l}$ & 50 & 100 & EPA Method 8270 \\
\hline
\end{tabular}




Analyte
Dissolved organic carbon
Disulfoton
Eh
Endosulfan I
Endosulfan II
Endosulfan sulfate
Endothall
Endrin
Endrin aldehyde
Endrin ketone
Ethylbenzene
Ethyl methacrylate
Ethyl methanesulfonate
Europium-152
Europium-154
Europium-155
Famphur
Fluoranthene
Fluorene
Fluoride
Glyphosate
Gross alpha
Heptachlor
Heptachlor epoxide
Heptachlorodibenzo-p-dioxin
isomers
$1,2,3,4,6,7,8-H P C D D$
Heptachlorodibenzo-p-furan
isomers
1,2,3,4,6,7,8-HPCDF
Hexachlorobenzene
Hexachlorobutadiene
Hexachlorocyclopentadiene
Hexachlorodibenzo-p-dioxin
isomers
1,2,3,4,7,8-HXCDD
Hexachlorodibenzo-p-furan
isomers
1,2,3,4,7,8-HXCDF
Hexachloroethane
Hexachlorophene
Hexachioropropene
2 2-Hexanone
Indeno[1,2,3-c,d]pyrene
lodine
lodine-129
lodine-131
lodomethane (Methyl iodide)
Iron
Iron, dissolved
iron, total recoverable
Iron-55
Iron-59
Isobutyl alcohol
Isodrin

\begin{tabular}{|c|c|c|c|}
\hline Unit & Flag 1 & Flag 2 & Source \\
\hline$\mu g / L$ & 5,000 & 10,000 & EPA Method 9060 \\
\hline$\mu g / L$ & 50 & 100 & EPA Method 8270 \\
\hline & No flag & No flag & Set by EPD/EMS \\
\hline$\mu \mathrm{g} / \mathrm{L}$ & 0.5 & 1 & EPA Method 8080 \\
\hline$\mu \mathrm{g} / \mathrm{L}$ & 0.5 & 1 & EPA Method 8080 \\
\hline$\mu g / L$ & 0.5 & 1 & EPA Method 8080 \\
\hline$\mu g / L$ & 50 & 100 & Final PDWS (EPA, 1993a) \\
\hline$\mu g / L$ & 1 & 2 & Final PDWS (EPA, 1993a) \\
\hline$\mu \mathrm{g} / \mathrm{L}$ & 0.5 & 1 & EPA Method 8080 \\
\hline & No flag & No flag & Set by EPD/EMS \\
\hline$\mu \mathrm{g} / \mathrm{L}$ & 350 & 700 & Final PDWS (EPA, 1993a) \\
\hline$\mu \mathrm{g} / \mathrm{L}$ & 50 & 100 & EPA Method 8270 \\
\hline$\mu \mathrm{g} / \mathrm{L}$ & 50 & 100 & EPA Method 8270 \\
\hline $\mathrm{PCi} / \mathrm{L}$ & $3 E+01$ & $6 \mathrm{E}+01$ & Interim Final PDWS (EPA, 1977) \\
\hline $\mathrm{pCi} / \mathrm{L}$ & $1 E+02$ & $2 E+02$ & Interim Final PDWS (EPA, 1977) \\
\hline $\mathrm{pCi} / \mathrm{L}$ & $3 E+02$ & $6 \mathrm{E}+02$ & Interim Final PDWS (EPA, 1977) \\
\hline$\mu \mathrm{g} / \mathrm{L}$ & 50 & 100 & EPA Method 8270 \\
\hline$\mu \mathrm{g} / \mathrm{L}$ & 50 & 100 & EPA Method 8270 \\
\hline$\mu \mathrm{g} / \mathrm{L}$ & 50 & 100 & EPA Method 8270 \\
\hline$\mu g / L$ & 2,000 & 4,000 & Final PDWS (EPA, 1993a) \\
\hline$\mu g / L$ & 350 & 700 & Final PDWS (EPA, 1993a) \\
\hline $\mathrm{pCi} / \mathrm{L}$ & $7.5 \mathrm{E}+00$ & $1.5 \mathrm{E}+01$ & Final PDWS (EPA, 1993a) \\
\hline$\mu \mathrm{g} / \mathrm{L}$ & 0.2 & 0.4 & Final PDWS (EPA, 1993a) \\
\hline$\mu g / L$ & 0.1 & 0.2 & Final PDWS (EPA, 1993a) \\
\hline$\mu g / L$ & 0.00325 & 0.0065 & EPA Method 8280 \\
\hline$\mu \mathrm{g} / \mathrm{L}$ & 0.00325 & 0.0065 & EPA Method 8280 \\
\hline$\mu g / L$ & 0.00225 & 0.0045 & EPA Method 8280 \\
\hline$\mu g / L$ & 0.00225 & 0.0045 & EPA Method 8280 \\
\hline$\mu \mathrm{g} / \mathrm{L}$ & 0.5 & 1 & Final PDWS (EPA, 1993a) \\
\hline$\mu g / L$ & 50 & 100 & EPA Method 8270 \\
\hline$\mu g / L$ & & & Final PDWS (EPA, 1993a) \\
\hline$\mu g / L$ & 0.00225 & 0.0045 & EPA Method 8280 \\
\hline$\mu g / L$ & 0.00225 & 0.0045 & EPA Method 8280 \\
\hline$\mu \mathrm{g} / \mathrm{L}$ & 0.002 & 0.004 & EPA Method 8280 \\
\hline$\mu \mathrm{g} / \mathrm{L}$ & 0.002 & 0.004 & EPA Method 8280 \\
\hline$\mu g / L$ & 50 & 100 & EPA Method 8270 \\
\hline$\mu g / L$ & 250 & 500 & EPA Method 8270 \\
\hline$\mu g / L$ & 50 & 100 & EPA Method 8270 \\
\hline$\mu g / L$ & 50 & 100 & EPA Method 8240 \\
\hline$\mu g / L$ & 50 & $\begin{array}{l}100 \\
500\end{array}$ & $\begin{array}{l}\text { EPA Method } 8270 \\
\text { APHA Method 415A }\end{array}$ \\
\hline $\begin{array}{l}\mu g / L \\
\mathrm{pCi} / \mathrm{L}\end{array}$ & $\begin{array}{l}250 \\
5 E-01\end{array}$ & $\begin{array}{l}500 \\
1 E+00\end{array}$ & \\
\hline $\begin{array}{l}\mathrm{pCi} / \mathrm{L} \\
\mathrm{pCi} / \mathrm{L}\end{array}$ & $\begin{array}{l}5 E-01 \\
1.5 E+00\end{array}$ & $\begin{array}{l}1 E+00 \\
3 E+00\end{array}$ & Interim Final PDWS (EPA, 1977) \\
\hline$\mu g / L$ & 75 & 150 & ÉPA Method 8240 \\
\hline$\mu \mathrm{g} / \mathrm{L}$ & 150 & 300 & SDWS (EPA, 1993b) \\
\hline$\mu g / L$ & 150 & 300 & SDWS (EPA, 1993b) \\
\hline$\mu g / L$ & 150 & 300 & SDWS (EPA, 1993b) \\
\hline pCi/L & $1 \mathrm{E}+03$ & $2 E+03$ & Interim Final PDWS (EPA, 1977) \\
\hline $\mathrm{pCi} / \mathrm{h}$ & $1 E+02$ & $2 E+02$ & Interim Final POWS (EPA, 1977) \\
\hline$\mu g / L$ & 500 & 1,000 & EPA Method 8240 \\
\hline & 50 & 100 & EPA Method 8270 \\
\hline
\end{tabular}




Analyte
Isophorone
Isosafrole
Kepone
Lanthanum-140
Lead
Lead, dissolved
Lead, total recoverable
Lead-212
Lindane
Lithium
Lithium, dissolved
Lithium, total recoverable
Magnesium
Magnesium, dissolved
Magnesium, total recoverable
Manganese
Manganese, dissolved
Manganese, total recoverable
Manganese-54
Mercury
Mercury, dissolved
Mercury, total recoverable
Methacrylonitrile
Methapyrilene
Methoxychlor
3-Methylcholanthrene
2-Methyl-4,6-dinitrophenol
Methyl ethyl ketone
Methyl isobutyl ketone
Methyl methacrylate
Methyl methanesulfonate
2-Methylnaphthalene
Molybdenum
Molybdenum, dissolved
Molybdenum, total recoverable
Naphthalene
1,4-Naphthoquinone
1-Naphthylamine
2-Naphthylamine
Neptunium-237
Nickel
Nickel, dissolved
Nickel, total recoverable
Nickel-59c
Nickel-63
Niobium-95
Nitrate as nitrogen
Nitrate-nitrite as nitrogen
Nitrite as nitrogen
m-Nitroaniline
o-Nitroaniline
p-Nitroaniline
Nitrobenzene
Nitrogen by Kjeldahl method
2-Nitrophenol.

\begin{tabular}{|c|c|c|}
\hline Unit & Flag 1 & Flag 2 \\
\hline$\mu \mathrm{g} / \mathrm{L}$ & 50 & 100 \\
\hline$\mu \mathrm{g} / \mathrm{L}$ & 50 & 100 \\
\hline$\mu \mathrm{g} / \mathrm{L}$ & 50 & 100 \\
\hline $\mathrm{pCi} / \Omega$ & $3 E+01$ & $6 \mathrm{E}+01$ \\
\hline$\mu g / L$ & 25 & 50 \\
\hline$\mu g / L$ & 25 & 50 \\
\hline$\mu g / L$ & 25 & 50 \\
\hline $\mathrm{pCi} / \mathrm{L}$ & $6.2 \mathrm{E}+01$ & $1.23 E+02$ \\
\hline$\mu \mathrm{g} / \mathrm{L}$ & 0.1 & 0.2 \\
\hline$\mu g / L$ & 25 & 50 \\
\hline$\mu g / L$ & 25 & 50 \\
\hline$\mu g / L$ & 25 & 50 \\
\hline & $\begin{array}{l}\text { No flag } \\
\text { No flag }\end{array}$ & $\begin{array}{l}\text { No flag } \\
\text { No flag }\end{array}$ \\
\hline & No flag & No flag \\
\hline$\mu g / L$ & 25 & 50 \\
\hline$\mu \mathrm{g} / \mathrm{L}$ & 25 & 50 \\
\hline$\mu g / L$ & 25 & 50 \\
\hline $\mathrm{pCi} / \mathrm{L}$ & $1.5 \mathrm{E}+02$ & $3 E+02$ \\
\hline$\mu \mathrm{g} / \mathrm{L}$ & 1 & 2 \\
\hline$\mu g / L$ & 1 & 2 \\
\hline$\mu g / L$ & 1 & 2 \\
\hline$\mu g / L$ & 250 & 500 \\
\hline$\mu \mathrm{g} / \mathrm{L}$ & 50 & 100 \\
\hline$\mu g / L$ & 20 & 40 \\
\hline$\mu \mathrm{g} / \mathrm{L}$ & 50 & 100 \\
\hline$\mu g / L$ & $\begin{array}{l}250 \\
\text { No flag }\end{array}$ & $\begin{array}{l}500 \\
\text { No flag }\end{array}$ \\
\hline & No flag & No flag \\
\hline$\mu g / L$ & 50 & 100 \\
\hline$\mu g / L$ & 50 & 100 \\
\hline$\mu \mathrm{g} / \mathrm{L}$ & 50 & 100 \\
\hline$\mu g / L$ & 250 & 500 \\
\hline$\mu g / L$ & 250 & 500 \\
\hline$\mu \mathrm{g} / \mathrm{L}$ & 250 & 500 \\
\hline$\mu g / L$ & 50 & 100 \\
\hline$\mu g / \mathrm{h}$ & 50 & 100 \\
\hline$\mu \mathrm{g} / \mathrm{L}$ & 50 & 100 \\
\hline$\mu g / L$ & 50 & 100 \\
\hline $\mathrm{pCi} / \mathrm{L}$ & $3.53 E+00$ & $7.06 \mathrm{E}+00$ \\
\hline$\mu g / L$ & 50 & 100 \\
\hline$\mu g / L$ & 50 & 100 \\
\hline$\mu g / L$ & 50 & 100 \\
\hline $\mathrm{pCi} / \mathrm{L}$ & $1.5 E+02$ & $3 E+02$ \\
\hline $\mathrm{pCi} / \mathrm{L}$ & $2.5 \mathrm{E}+01$ & $5 E+01$ \\
\hline $\mathrm{pCi} / \mathrm{L}$ & $1.5 E+02$ & $3 . E+02$ \\
\hline$\mu g / L$ & 5,000 & 10,000 \\
\hline$\mu g / L$ & 5,000 & 10,000 \\
\hline$\mu g / L$ & 500 & 1,000 \\
\hline$\mu g / L$ & 50 & 100 \\
\hline$\mu \mathrm{g} / \mathrm{L}$ & 50 & 100 \\
\hline$\mu g / L$ & 50 & 100 \\
\hline$\mu g / L$ & 50 & 100 \\
\hline$\mu g / L$ & 500 & 1,000 \\
\hline$\mu g / L$ & 50 & 100 \\
\hline
\end{tabular}

Source

EPA Method 8270

EPA Method 8270

EPA Method 8270

Interim Final PDWS (EPA, 1977)

Final PDWS (SCDHEC, 1981)

Final PDWS (SCDHEC, 1981)

Final PDWS (SCDHEC, 1981)

Proposed PDWS (EPA, 1991)

Final PDWS (EPA, 1993a)

EPA Method 6010

EPA Method 6010

EPA Method 6010

Set by EPD/EMS

Set by EPD/EMS

Set by EPD/EMS

SDWS (EPA, 1993b)

SDWS (EPA, 1993b)

SDWS (EPA, 1993b)

Interim Final PDWS (EPA, 1977)

Final PDWS (EPA, 1993a)

Final PDWS (EPA, 1993a)

Final PDWS (EPA, 1993a)

EPA Method 8240

EPA Method 8270

Final PDWS (EPA, 1993a)

EPA Method 8270

EPA Method 8270

Set by EPD/EMS

Set by EPD/EMS

EPA Method 8270

EPA Method 8270

EPA Method 8270

EPA Method 6010

EPA Method 6010

EPA Method 6010

EPA Method 8270

EPA Method 8270

EPA Method 8270

EPA Method 8270

Proposed PDWS (EPA, 1991)

Final PDWS (EPA, 1993a)

Final PDWS (EPA, 1993a)

Final PDWS (EPA, 1993a)

Interim Final PDWS (EPA, 1977)

Interim Final PDWS (EPA, 1977)

Interim Final PDWS (EPA, 1977)

Final PDWS (EPA, 1993a)

Final PDWS (EPA, 1993a)

Final PDWS (EPA, 1993a)

EPA Method 8270

EPA Method 8270

EPA Method 8270

EPA Method 8270

EPA Method 351.2

EPA Method 8270 


Analyte
4-Nitrophenol
4-Nitroquinoline-1-oxide
N-Nitrosodi-n-butylamine
N-Nitrosodiethylamine
N-Nitrosodimethylamine
N-Nitrosodiphenylamine
N-Nitrosodipropylamine
N-Nitrosomethylethylamine
N-Nitrosomorpholine
N-Nitrosopiperidine
N-Nitrosopyrrolidine
5-Nitro-o-toluidine
Nonvolatile beta
Octachlorodibenzo-p-dioxin
isomers
Octachlorodibenzo-p-furan
isomers
Odor
Oil \& Grease
Oxamyb
Parathion
Parathion methyl
PCB 1016
PCB 1221
PCB 1232
PCB 1242
PCB 1248
PCB 1254
PCB 1260
PCB 1262
Pentachlorobenzene
Pentachlorodibenzo-p-dioxin
isomers
1,2,3,7,8-PCDD
Pentachlorodibenzo-p-furan
isomers
1,2,3,7,8-PCDF
Pentachloroethane
Pentachloronitrobenzene
Pentachlorophenol
pH
pH
Phenacetin
Phenanthrene
Phenol
Phenols
p-Phenylenediamine
Phorate
Picloram
2-Picoline
Plutonium-238
Plutonium-239
Plutonium-239/240
Plutonium-240
Plutonium-241

\begin{tabular}{|c|c|c|c|}
\hline Unit & Flag 1 & Flag 2 & Source \\
\hline$\mu g / L$ & 50 & 100 & EPA Method 8270 \\
\hline$\mu g / L$ & 50 & 100 & EPA Method 8270 \\
\hline$\mu \mathrm{g} / \mathrm{L}$ & 50 & 100 & EPA Method 8270 \\
\hline$\mu g / L$ & 50 & 100 & EPA Method 8270 \\
\hline$\mu \mathrm{g} / \mathrm{L}$ & 50 & 100 & EPA Method 8270 \\
\hline$\mu g / L$ & 50 & 100 & EPA Method 8270 \\
\hline$\mu g / L$ & 50 & 100 & EPA Method 8270 \\
\hline$\mu \mathrm{g} / \mathrm{L}$ & 50 & 100 & EPA Method 8270 \\
\hline$\mu \mathrm{g} / \mathrm{L}$ & 50 & 100 & EPA Method 8270 \\
\hline$\mu \mathrm{g} / \mathrm{L}$ & 50 & 100 & EPA Method 8270 \\
\hline$\mu g / L$ & 50 & 100 & EPA Method 8270 \\
\hline$\mu \mathrm{g} / \mathrm{L}$ & 50 & 100 & EPA Method 8270 \\
\hline $\mathrm{pCi} / \mathrm{L}$ & $2.5 \mathrm{E}+01$ & $5 E+01$ & Interim Final PDWS (EPA, 1977) \\
\hline$\mu g / L$ & 0.005 & 0.01 & EPA Method 8280 \\
\hline$\mu \mathrm{g} / \mathrm{L}$ & 0.005 & 0.01 & EPA Method 8280 \\
\hline & No flag & No flag & Set by EPD/EMS \\
\hline$\mu \mathrm{g} / \mathrm{L}$ & 5,000 & 10,000 & EPA Method 413.1 \\
\hline$\mu \mathrm{g} / \mathrm{L}$ & 100 & 200 & Final PDWS (EPA, 1993a) \\
\hline$\mu g / L$ & 0.25 & 0.5 & EPA Method 8080 \\
\hline$\mu g / L$ & 0.25 & 0.5 & EPA Method 8080 \\
\hline$\mu g / L$ & 0.25 & 0.5 & Final PDWS (EPA, 1993a) \\
\hline$\mu g / L$ & 0.25 & 0.5 & Final PDWS (EPA, 1993a) \\
\hline$\mu g / L$ & 0.25 & 0.5 & Final PDWS (EPA, 1993a) \\
\hline$\mu \mathrm{g} / \mathrm{L}$ & 0.25 & 0.5 & Final PDWS (EPA, 1993a) \\
\hline$\mu \mathrm{g} / \mathrm{L}$ & 0.25 & 0.5 & Final PDWS (EPA, 1993a) \\
\hline$\mu \mathrm{g} / \mathrm{L}$ & 0.25 & 0.5 & Final PDWS (EPA, 1993a) \\
\hline$\mu g / L$ & 0.25 & 0.5 & Final PDWS (EPA, 1993a) \\
\hline$\mu \mathrm{g} / \mathrm{L}$ & 0.25 & 0.5 & Final PDWS (EPA, 1993a) \\
\hline$\mu \mathrm{g} / \mathrm{L}$ & & 100 & EPA Method 8270 \\
\hline$\mu g / L$ & 0.00275 & 0.0055 & EPA Method 8280 \\
\hline$\mu g / L$ & 0.00275 & 0.0055 & EPA Method 8280 \\
\hline$\mu g / L$ & 0.00275 & 0.0055 & EPA Method 8280 \\
\hline$\mu g / L$ & 0.00275 & 0.0055 & EPA Method 8280 \\
\hline$\mu g / L$ & 50 & 100 & EPA Method 8270 \\
\hline$\mu g / L$ & 50 & 100 & EPA Method 8270 \\
\hline$\mu g / L$ & 0.5 & 1 & Final PDWS (EPA, 1993a) \\
\hline $\mathrm{pH}$ & 8 & 10 & Set by EPD/EMS \\
\hline $\mathrm{pH}$ & 4 & 3 & Set by EPD/EMS \\
\hline$\mu g / L$ & 50 & 100 & EPA Method 8270 \\
\hline$\mu \mathrm{g} / \mathrm{L}$ & 50 & 100 & EPA Method 8270 \\
\hline$\mu g / L$ & 50 & 100 & EPA Method 8270 \\
\hline$\mu \mathrm{g} / \mathrm{L}$ & 25 & 50 & EPA Method 420.1 \\
\hline$\mu \mathrm{g} / \mathrm{L}$ & 50 & 100 & EPA Method 8270 \\
\hline$\mu \mathrm{g} / \mathrm{L}$ & 0.5 & 1 & EPA Method 8080 \\
\hline$\mu \mathrm{g} / \mathrm{L}$ & 250 & 500 & Final PDWS (EPA, 1993a) \\
\hline$\mu g / L$ & 50 & 100 & EPA Method 8270 \\
\hline $\mathrm{pCi} / \mathrm{L}$ & $3.51 \mathrm{E}+00$ & $7.02 \mathrm{E}+00$ & Proposed PDWS (EPA, 1991) \\
\hline $\mathrm{pCi} / \mathrm{L}$ & $3.11 \mathrm{E}+01$ & $6.21 \mathrm{E}+01$ & Proposed PDWS (EPA, 1991) \\
\hline $\mathrm{pCi} / \mathrm{L}$ & $3.11 \mathrm{E}+01$ & $6.21 E+01$ & Proposed PDWS (EPA, 1991) \\
\hline $\mathrm{pCi} / \mathrm{L}$ & $3.11 E+01$ & $6.22 E+01$ & Proposed PDWS (EPA, 1991) \\
\hline $\mathrm{pCi} / \mathrm{L}$ & $3.13 \mathrm{E}+01$ & $6.26 E+01$ & Proposed PDWS (EPA, 1991) \\
\hline
\end{tabular}




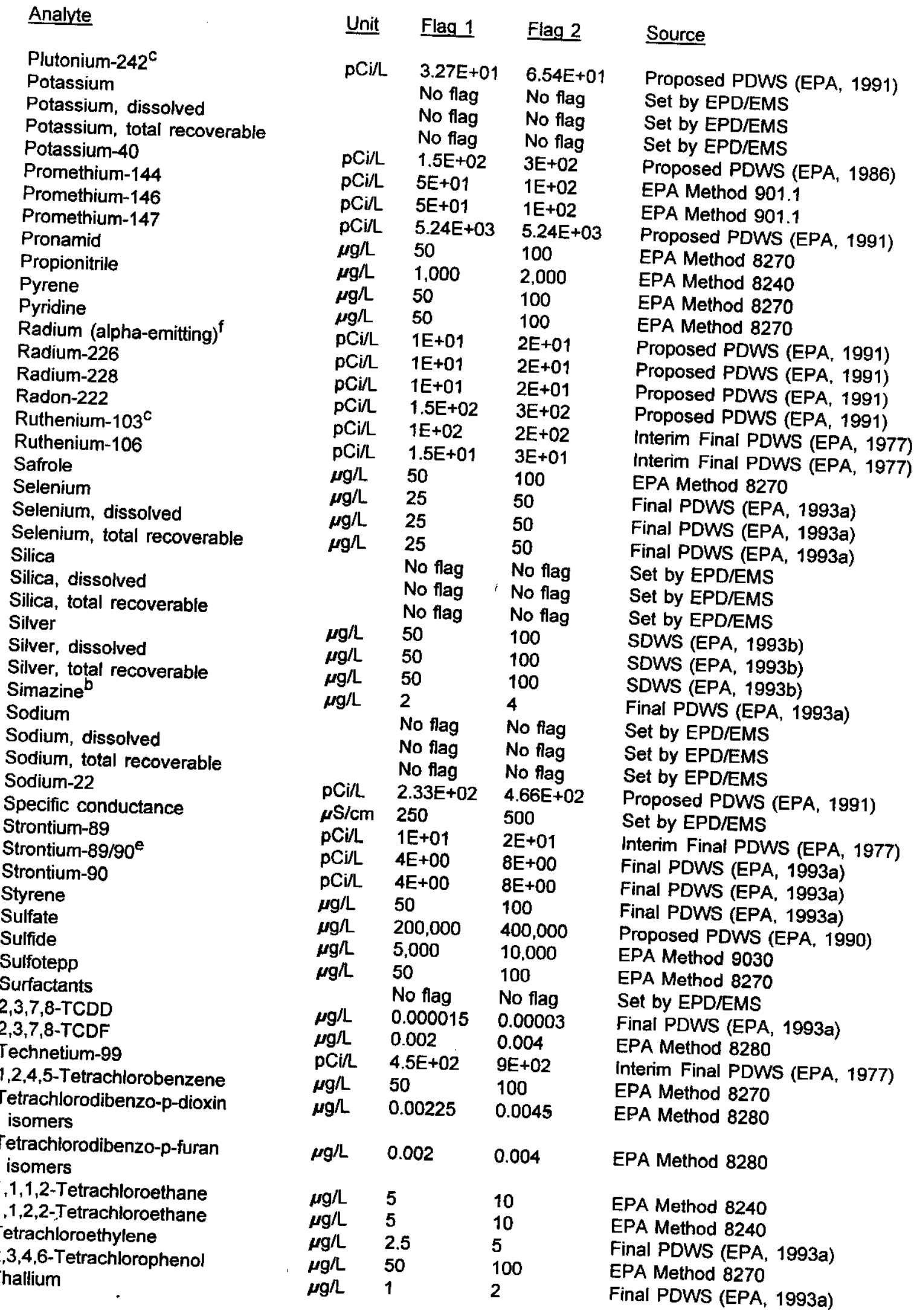




Analyte
Thallium, dissolved
Thallium, total recoverable
Thionazin
Thorium-228
Thorium-230
Thorium-232
Thorium-234
Tin
Tin, dissolved
Tin, total recoverable
Tin-113
Toluene
o-Toluidine
Total carbon
Total coliform
Total dissolved solids
Total hydrocarbons
Total inorganic carbon
Total organic carbon
Total organic halogens
Total organic nitrogen
Total petroleum hydrocarbons
Total phosphates (as P)
Total phosphorus
Toxaphene
2,4,5-TP (Silvex)
Tributyl phosphate
1,2,4-Trichlorobenzene
1,1,1-Trichloroethane
1,1,2-Trichloroethane
Trichloroethylene
Trichlorofluoromethane
2,4,5-Trichlorophenol
2,4,6-Trichlorophenol
$2,4,5-$-Trichlorophenoxyacetic acid
1,2,3-Trichloropropane
0,0,O-Triethyl phosphorothioate
1,3,5-Trinitrobenzene
Tritium
Turbidity
Uranium
Uranium, dissolved
Uranium, total recoverable
Uranium alpha activity
Uranium-233/234
Uranium-234
Uranium-235
Uranium-238
Vanadium
Vanadium, dissolved
Vanadium, total recoverable
Vinyl acetate

\begin{tabular}{|c|c|c|}
\hline Unit & Flag 1 & Flag 2 \\
\hline$\mu \mathrm{g} / \mathrm{L}$ & 1 & 2 \\
\hline$\mu g / L$ & 1 & 2 \\
\hline$\mu g / L$ & 50 & 100 \\
\hline $\mathrm{pCi} / \mathrm{L}$ & $6.25 E+01$ & $1.25 E+02$ \\
\hline $\mathrm{pCi} / \mathrm{L}$ & $3.96 \mathrm{E}+01$ & $7.92 E+01$ \\
\hline pCi/L & 4.4E+01 & $8.8 E+01$ \\
\hline $\mathrm{pCi} / \mathrm{L}$ & $2 E+02$ & $4.01 E+02$ \\
\hline$\mu \mathrm{g} / \mathrm{L}$ & 10 & 20 \\
\hline$\mu \mathrm{g} / \mathrm{L}$ & 10 & 20 \\
\hline$\mu \mathrm{g} / \mathrm{L}$ & 10 & 20 \\
\hline $\mathrm{pCi} / \mathrm{L}$ & $1.5 E+02$ & $3 E+02$ \\
\hline$\mu \mathrm{g} / \mathrm{L}$ & 500 & 1,000 \\
\hline$\mu \mathrm{g} / \mathrm{L}$ & 50 & 100 \\
\hline$\mu g / L$ & 5,000 & 10,000 \\
\hline & $\begin{array}{l}0 \\
\text { No flag }\end{array}$ & $\begin{array}{l}0 \\
\text { No flag }\end{array}$ \\
\hline$\mu g / L$ & 5,000 & 10,000 \\
\hline$\mu \mathrm{g} / \mathrm{L}$ & 5,000 & 10,000 \\
\hline$\mu g / L$ & 5,000 & 10,000 \\
\hline$\mu g / L$ & 25 & 50 \\
\hline$\mu \mathrm{g} / \mathrm{L}$ & 500 & 1,000 \\
\hline$\mu g / L$ & 5,000 & 10,000 \\
\hline & $\begin{array}{l}\text { No flag } \\
\text { No flag }\end{array}$ & $\begin{array}{l}\text { No flag } \\
\text { No flag }\end{array}$ \\
\hline$\mu g / L$ & 1.5 & 3 \\
\hline$\mu \mathrm{g} / \mathrm{L}$ & 25 & 50 \\
\hline$\mu \mathrm{g} / \mathrm{L}$ & 50 & 100 \\
\hline$\mu g / L$ & 35 & 70 \\
\hline$\mu g / L$ & 100 & 200 \\
\hline$\mu \mathrm{g} / \mathrm{L}$ & 2.5 & 5 \\
\hline$\mu \mathrm{g} / \mathrm{L}$ & 2.5 & 5 \\
\hline$\mu \mathrm{g} / \mathrm{L}$ & 5 & 10 \\
\hline$\mu g / L$ & 50 & 100 \\
\hline$\mu g / L$ & 50 & 100 \\
\hline$\mu g / L$ & 2.5 & 5 \\
\hline$\mu g / L$ & 5 & 10 \\
\hline$\mu \mathrm{g} / \mathrm{L}$ & 50 & 100 \\
\hline$\mu \mathrm{g} / \mathrm{L}$ & 50 & 100 \\
\hline $\mathrm{pCi} / \mathrm{mL}$ & $\begin{array}{l}1 E+01 \\
\text { No flag }\end{array}$ & $\begin{array}{l}2 E+01 \\
\text { No flag }\end{array}$ \\
\hline$\mu g / L$ & 10 & 20 \\
\hline$\mu g / L$ & 10 & 20 \\
\hline$\mu g / L$ & 10 & 20 \\
\hline $\mathrm{pCi} / \mathrm{L}$ & $1.5 \mathrm{E}+01$ & $3 E+01$ \\
\hline $\mathrm{pCi} / \mathrm{L}$ & $6.9 \mathrm{E}+00$ & $1.38 \mathrm{E}+01$ \\
\hline $\mathrm{Ci} / \mathrm{L}$ & $6.95 \mathrm{E}+00$ & $1.39 E+01$ \\
\hline $\mathrm{Ci} / \mathrm{L}$ & $7.25 \mathrm{E}+00$ & $1.45 \mathrm{E}+01$ \\
\hline Ci/L & $7.3 E+00$ & $1.46 \mathrm{E}+01$ \\
\hline 97 & 40 & 80 \\
\hline $\mathrm{g} / \mathrm{h}$ & 40 & 80 \\
\hline g/l & 40 & 80 \\
\hline $\mathrm{g} / \mathrm{h}$ & 5 & 10 \\
\hline
\end{tabular}

\section{Source}

Final PDWS (EPA, 1993a)

Final PDWS (EPA, 1993a)

EPA Method 8270

Proposed PDWS (EPA, 1991)

Proposed PDWS (EPA, 1991)

Proposed PDWS (EPA, 1991)

Proposed PDWS (EPA, 1991)

EPA Method 282.2

EPA Method 282.2

EPA Method 282.2

Interim Final PDWS (EPA, 1977)

Final PDWS (EPA, 1993a)

EPA Method 8270

EPA Method 9060

Final PDWS (EPA, 1993a)

Set by EPD/EMS

EPA Method 418.1

EPA Method 9060

EPA Method 9060

EPA Method 9020

APHA Method 420

EPA Method 418.1

Set by EPD/EMS

Set by EPD/EMS

Final PDWS (EPA, 1993a)

Final PDWS (EPA, 1993a)

EPA Method 8270

Final PDWS (EPA, 1993a)

Final PDWS (EPA, 1993a)

Final PDWS (EPA, 1993a)

Final PDWS (EPA, 1993a)

EPA Method 8240

EPA Method 8270

EPA Method 8270

EPA Method 8150

EPA Method 8240

EPA Method 8270

EPA Method 8270

Final PDWS (EPA, 1993a)

Set by EPD/EMS

Proposed PDWS (EPA, 1991)

Proposed PDWS (EPA, 1991)

Proposed PDWS (EPA, 1991)

Proposed PDWS (EPA, 1991)

Proposed PDWS (EPA, 1991)

Proposed PDWS (EPA, 1991)

Proposed PDWS (EPA, 1991)

Proposed PDWS (EPA, 1991)

EPA Method 6010

EPA Method 6010

EPA Method 6010

EPA Method 8240 


\begin{tabular}{|c|c|c|c|c|}
\hline Analyte & Unit & Flag 1 & Flag 2 & Source \\
\hline Xylenes & $\mu g / L$ & 5,000 & 10,000 & Final PDWS (EPA, 1993a) \\
\hline Yttrium-88 & $\mathrm{pCi} / \mathrm{L}$ & $5 E+01$ & $1 E+02$ & EPA Method 901.1 \\
\hline Zinc & $\mu \mathrm{g} / \mathrm{L}$ & 2,500 & 5,000 & SDWS (EPA, 1993b) \\
\hline Zinc, dissolved & $\mu g / L$ & 2,500 & 5,000 & SDWS (EPA, 1993b) \\
\hline Zinc, total recoverable & $\mu g / L$ & 2,500 & 5,000 & SDWS (EPA, 1993b) \\
\hline Zinc-65 & $\mathrm{pCi} / \mathrm{L}$ & $1.5 E+02$ & $3 E+02$ & Interim Final PDWS (EPA, 1977) \\
\hline Zirconium-95 & $\mathrm{pCi} / \mathrm{L}$ & $1 E+02$ & $2 E+02$ & Interim Final PDWS (EPA, 1977) \\
\hline Zirconium/Niobium-95 ${ }^{\mathrm{C}}$ & $\mathrm{pCi} / \mathrm{L}$ & $1 \mathrm{E}+02$ & $2 E+02$ & Interim Final PDWS (EPA, 1977) \\
\hline
\end{tabular}

a References for methods are in Appendix E; references for dated sources are at the end of this appendix.

b EMS is currently unable to perform this analysis.

c EMS discontinued monitoring this radionuclide because it is inappropriate for the SRS Groundwater Monitoring Program.

d EPD/EMS set this flagging criterion using the 1991 proposed PDWS because the final PDWS in 1977 may have been in error.

e For double radionuclide analyses where each separate radionuclide has its own standard, the more stringent standard is used.

$f$ The applied standard is for radium-226.

9 The primary maximum contaminant level range for turbidity is $1-5 \mathrm{NTU}$, which is inappropriate for the SRS Groundwater Monitoring Program.

\section{References}

EPA (U.S. Environmental Protection Agency), 1977. National Interim Primary Drinking Water Regulations, EPA-570/9-76-003. Washington, DC.

EPA (U.S. Environmental Protection Agency), 1986. Water Pollution Control; National Primary Drinking Water Regulations, Radionuclides (Proposed). Federal Register, September 30, 1986, pp. 34835-34862. Washington, DC.

EPA (U.S. Environmental Protection Agency), 1990. National Primary and Secondary Drinking Water Regulations; Synthetic Organic Chemicals and Inorganic Chemicals (Proposed Rule). Federal Register, July 25, 1990, pp. 30369-30448. Washington, DC.

EPA (U.S. Environmental Protection Agency), 1991. National Primary Drinking Water Regulations; Radionuclides; Proposed Rule. Federal Register, July 18, 1991, pp. 33052-33127. Washington, DC.

EPA (U.S. Environmental Protection Agency), 1993a. National Primary Drinking Water Regulations. Code of Federal Regulations, Section 40, Part 141, pp. 592-732. Washington, DC.

EPA (U.S. Environmental Protection Agency), 1993b. National Secondary Drinking Water Regulations. Code of Federal Regulations, Section 40, Part 143, pp. 774-777. Washington, DC.

SCDHEC (South Carolina Department of Health and Environmental Control), 1981. State Primary Drinking Water Regulations, R.61-58.5. Columbia, SC. 


\section{Appendix C}

Figures 
WSRC-TR-94-0347

Unclassified

THIS PAGE LEFT BLANK INTENTIONALLY. 


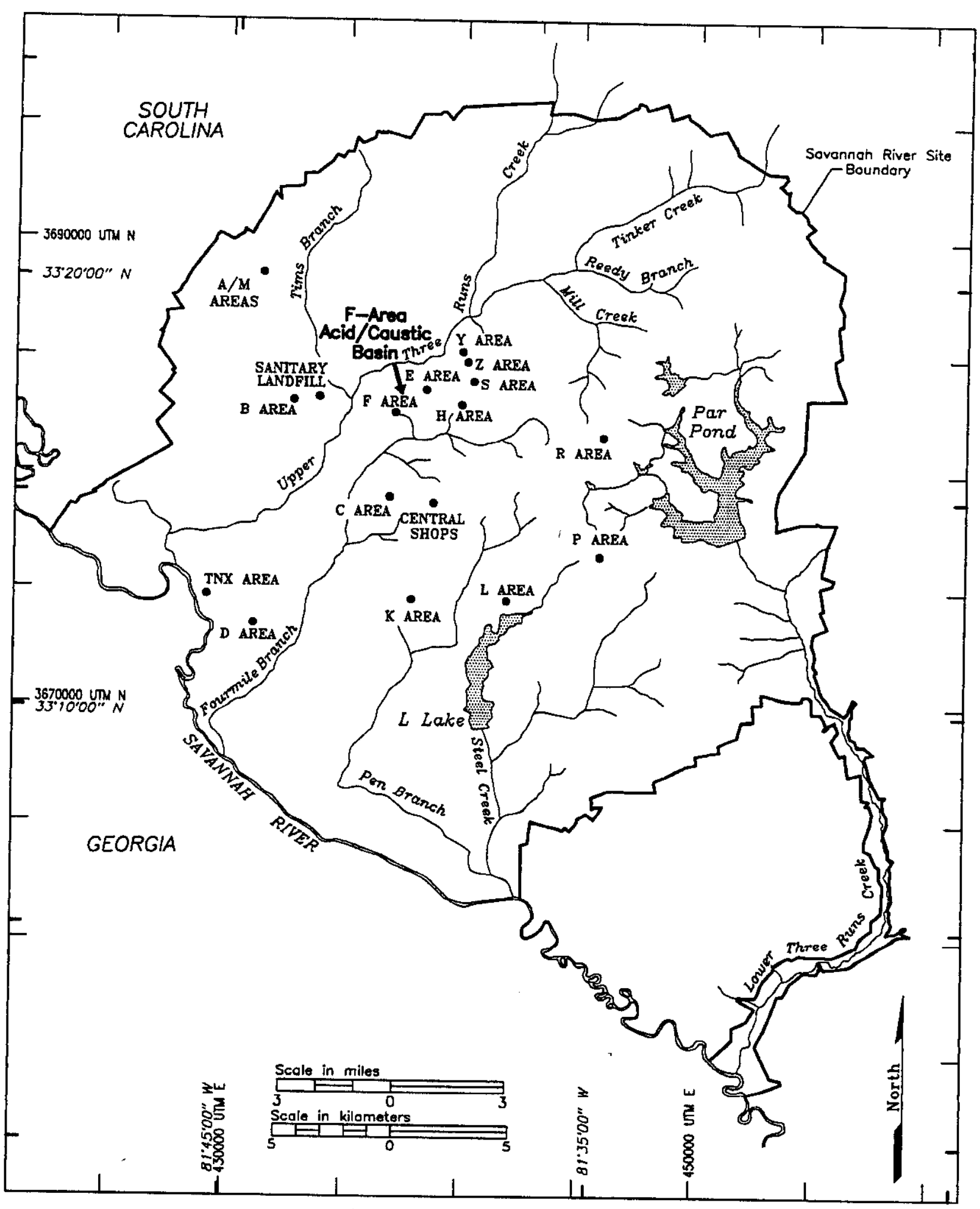

Figure 1. Location of the F-Area Acid/Caustic Basin at the Savannah River Site 


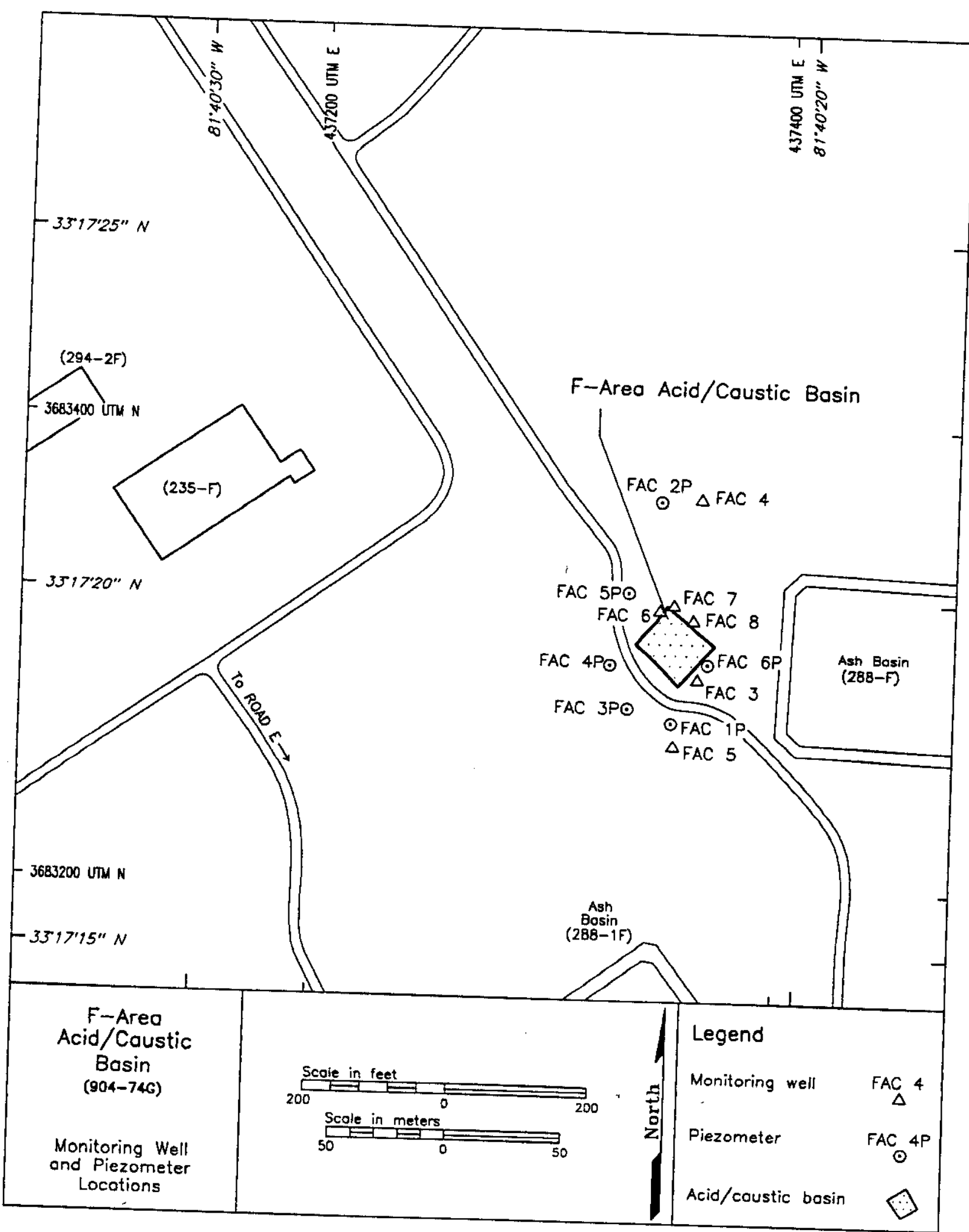

Figure 2. Location of Groundwater Monitoring Wells and Piezometer Wells at the F-Area
Acid/Caustic Basin 


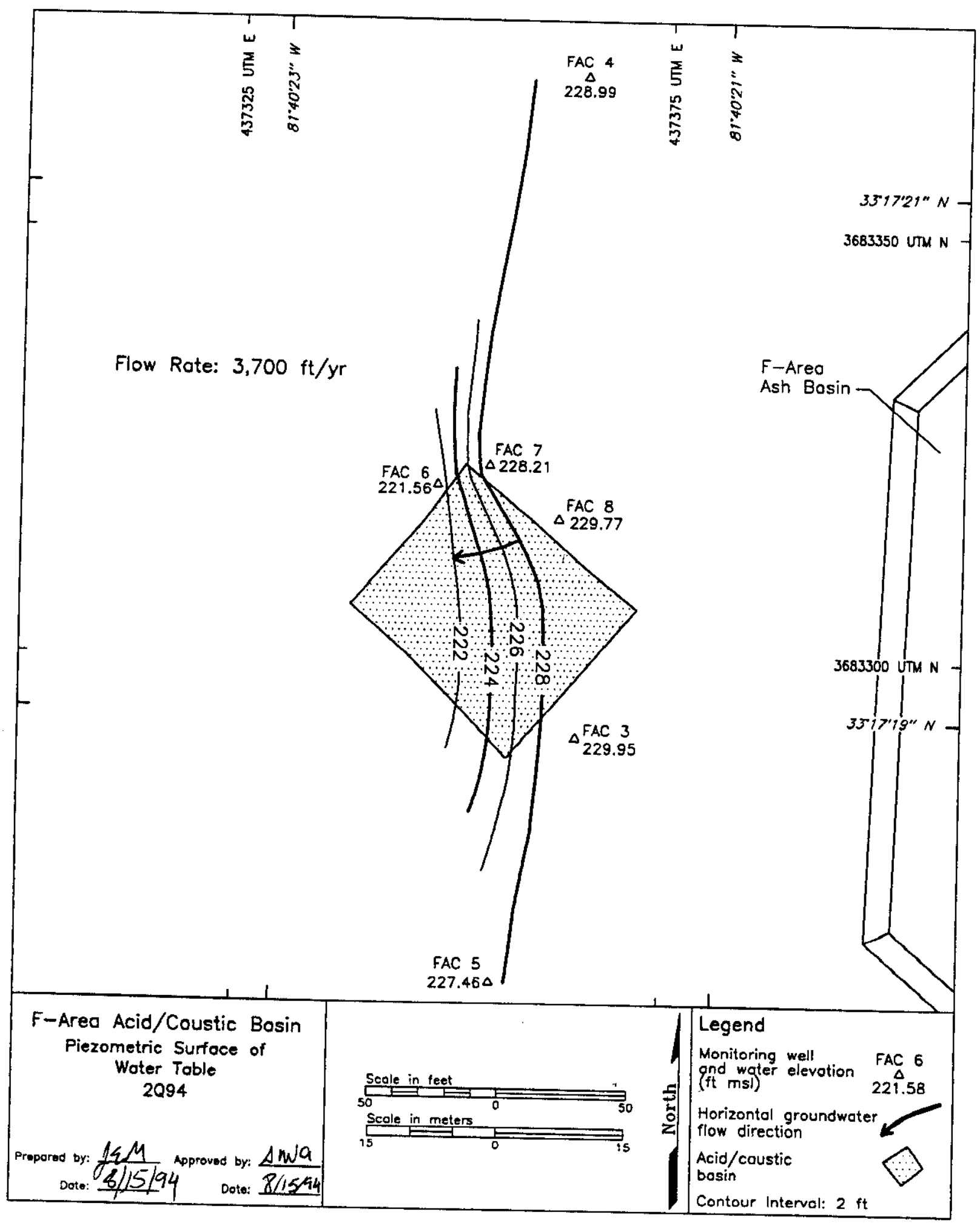
Figure 3. Piezometric Surface Map of the Water Table at the F-Area Acid/Caustic Basin,
Monitoring Well Data 


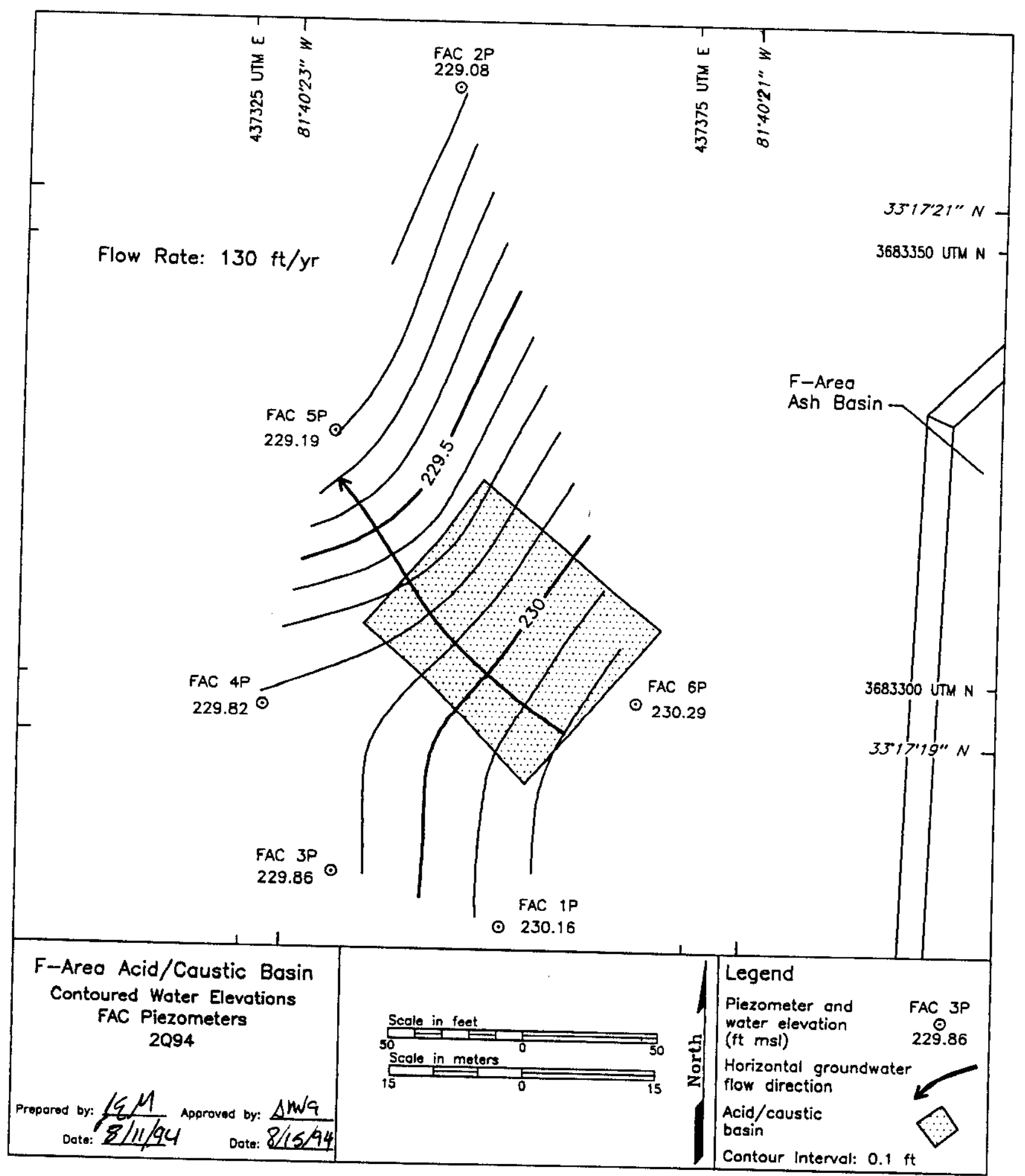

Figure 4. Water-Elevation Contour Map of the Water Table at the F-Area Acid/Caustic Basin,
Piezometer Data 


\section{Appendix D}

\section{Groundwater Monitoring Results Tables}


WSRC-TR-94-0347

Unclassified

THIS PAGE LEFT BLANK INTENTIONALLY. 


\section{Key to Reading the Tables}

The following abbreviations may appear in the data tables:

\section{Constituents}

\section{1,2,3,4,6,7,8-HPCDD \\ 1,2,3,4,6,7,8-HPCDF \\ $1,2,3,4,7,8-\mathrm{HXCDD}$ \\ $1,2,3,4,7,8-\mathrm{HXCDF}$ \\ Lindane \\ PCB}

1,2,3,7,8-PCDD

1,2,3,7,8-PCDF

Sp. conductance

TCDD

TCDF

\section{Laboratories}

\section{CN \\ EM}

GE and GP

SC

SP

TM

WA and WS

\section{Sampling Codes}

\author{
B \\ C \\ D \\ E \\ I \\ L \\ $\mathrm{P}$ \\ $s$ \\ $\mathrm{X}$
}

\section{Sampling Methods}
V

B

$P$

S 1,2,3,4,6,7,8-heptachlorodibenzo-p-dioxin

$1,2,3,4,6,7,8$-heptachlorodibenzo-p-furan

$1,2,3,4,7,8$-hexachlorodibenzo-p-dioxin

$1,2,3,4,7,8$-hexachlorodibenzo-p-furan

gamma-benzene hexachloride

polychlorinated biphenyl

1,2,3,7,8-pentachlorodibenzo-p-dioxin

1,2,3,7,8-pentachlorodibenzo-p-furan

specific conductance

tetrachlorodibenzo-p-dioxin

tetrachlorodibenzo-p-furan
Clemson Technical Center, Inc.

Environmental Protection Department/Environmental Monitoring Section (EPD/EMS) Laboratory

General Engineering Laboratories

Savannah River Technology Center

Spencer Testing Services, Inc.

TMA/Eberline

Roy F. Weston, Inc. blank sample was collected

well was pumping continuously

well was dry

equipment blank was collected

well went dry during sampling; insufficient water to collect all samples

well went dry before sampling began; only depth to water can be determined

inaccessibility or mechanical failure prevented sample

collection and field analysis of the water

no water in standpipe; for water level events only

well went dry during purging; samples collected after well

recovered sample collected using an open-bucket bailer sample collected using a bladder pump sample collected using a single-speed centrifugal downhole pump sample collected using a variable-speed pump 
Units

E

$\mathrm{mg} / \mathrm{L}$

msl

MSL

NTU

$\mathrm{pCi} / \mathrm{L}$

$\mathrm{pCi} / \mathrm{mL}$

$\mathrm{pH}$

$\mu \mathrm{g} / \mathrm{L}$

$\mu \mathrm{S} / \mathrm{cm}$

Other

CS

D

DF

GS

H

Mod

PDWS

PVC

TOC exponential notation (e.g., $1.1 \mathrm{E}-0.9=1.1 \times 10^{-9}=$ 0.0000000011 )

milligrams per liter

mean sea level

million structures per liter

turbidity unit

picocuries per liter

picocuries per milliliter

$\mathrm{pH}$ unit

micrograms per liter

microsiemens per centimeter

carbon steel

exceeded final Primary Drinking Water Standard

(PDWS) or screening level column in data tables

dilution factor column in data tables

groundwater protection standard column in data tables

holding time column in data tables

modifier column in data tables

primary drinking water standard

polyvinyl chloride

top of casing

\section{Holding Times}

Standard analytical methods include a limit, called holding time, on the maximum elapsed time large bullet $(\cdot)$ in the $H$ (holding time) column indicates that holding time was data tables, a Analyses performed beyond holding times may not yield valid results.

The South Carolina Department of Health and Environmental Control allows only 15 minutes to holding time criterion; laboratory $\mathrm{pH}$ an for $\mathrm{pH}$. Thus, only field $\mathrm{pH}$ measurements can meet the The laboratory procedure used for the determination of specific conductance allows one day to
elapse between sampling and analysis. Thus, laboratory specific conductance measurements
may exceed the holding time criterion.

\section{Data Rounding}

Constituent results in analytical results tables that appear to equal the final PDWS but are not in the database. Values stored in the database contain level) column are below the final PDWS results. Apparent discrepancies in the tables are due to the significant digits than the reported 


\section{Data Qualification}

The contract laboratories continually assess their own accuracy and precision according to U.S. Environmental Protection Agency (EPA) guidelines. They submit sample- or batch-specific qualterly summary. Properly defined and either at the same time as analytical results or in a quara key component in assessing data used result modifiers (also referred to as qualifiers) can be Protection Department/Environmental Meability. Result modifiers designed by the Environmental are defined below. These modifiers appear ing Section and provided to the primary laboratories tered modifiers are based on EPA's STORET the data tables under the column Mod. The let-

\section{Result modifier}

Data are not qualified. Numbers should be interpreted exactly as reported.

Value is estimated because quantitation in the sample or in associated quality control samples did not meet specifications.

The value in the result field is the instrument reading, not the sample quantification limit. Always used with the result qualifier $U$.

Value is off-scale high. The actual value is not known but is known to be greater than the value shown.

Presence of the analyte is verified but not quantified.

Result was rejected because performance requirements in the sample analysis or associated quality control analyses were not met

Analyte was not detected; if present, it was below the criteria for detection.

Material analyzed for but not detected. Analytical result reported is less than the sample quantitation limit.

Analyte was detected in an associated method blank.

Result was obtained from an unpreserved or improperly preserved sample. Data may not be accurate.

Result may be an underestimation of the true value due to analytical bias.

Result may be an overestimation of the true value due to analytical bias.

The associated result may be of poor precision (high variability) due to analytical bias.

Result is associated with QA results indicating matrix interference.

The associated result is from a reanalysis performed out of holding time due to problems with an earlier analysis. 
Table 1. Maximum Results for Constituents Exceeding Final Primary Drinking Water Standards

\begin{tabular}{|c|c|c|c|c|c|c|}
\hline Well & Constituent & Unit & $\underline{3 Q 93}$ & $\underline{4 Q 93}$ & $\underline{1094}$ & 2Q94 \\
\hline FAC 3 & $\begin{array}{l}\text { Dichloromethane } \\
\text { Gross alpha } \\
\text { Nonvolatile beta }\end{array}$ & $\begin{array}{l}\mu \mathrm{g} / \mathrm{L} \\
\mathrm{pCi} / \mathrm{L} \\
\mathrm{pCi} / \mathrm{L}\end{array}$ & $\begin{array}{l}9.4 \\
3.9 E+01 \\
-\end{array}$ & $\begin{array}{l}-^{a} \\
- \\
-\end{array}$ & $\begin{array}{l}- \\
7.2 E+01 \\
6.8 E+01\end{array}$ & $\begin{array}{l}- \\
2.3 E+01 \\
-\end{array}$ \\
\hline FAC 4 & $\begin{array}{l}\text { Dichloromethane } \\
\text { Gross alpha } \\
\text { Nonvolatile beta }\end{array}$ & $\begin{array}{l}\mu \mathrm{g} / \mathrm{L} \\
\mathrm{pCi} / \mathrm{L} \\
\mathrm{pCi} / \mathrm{L}\end{array}$ & $\begin{array}{l}12 \\
3.4 E+01 \\
-\end{array}$ & $\begin{array}{l}- \\
4.7 E+01 \\
-\end{array}$ & $\begin{array}{l}-\overline{6.6 E+01} \\
5.5 E+01\end{array}$ & $\begin{array}{l}- \\
- \\
-\end{array}$ \\
\hline FAC 5 & $\begin{array}{l}\text { Dichloromethane } \\
\text { Gross alpha }\end{array}$ & $\begin{array}{l}\mu g / L \\
p C i / L\end{array}$ & $\begin{array}{l}6.2 \\
3.2 E+01\end{array}$ & $\overline{3.8 E}+01$ & $\overline{4.8 E}+01$ & - \\
\hline FAC 8 & Dichloromethane & $\mu g / L$ & 5.5 & - & - & - \\
\hline
\end{tabular}

Note: The modifier column applies to second quarter 1994 data only.

a $-=$ not above final PDWS.

Table 2. Maximum Results for Constituents Exceeding Other Flag 2 Criteria or the SRS Turbidity
Standard

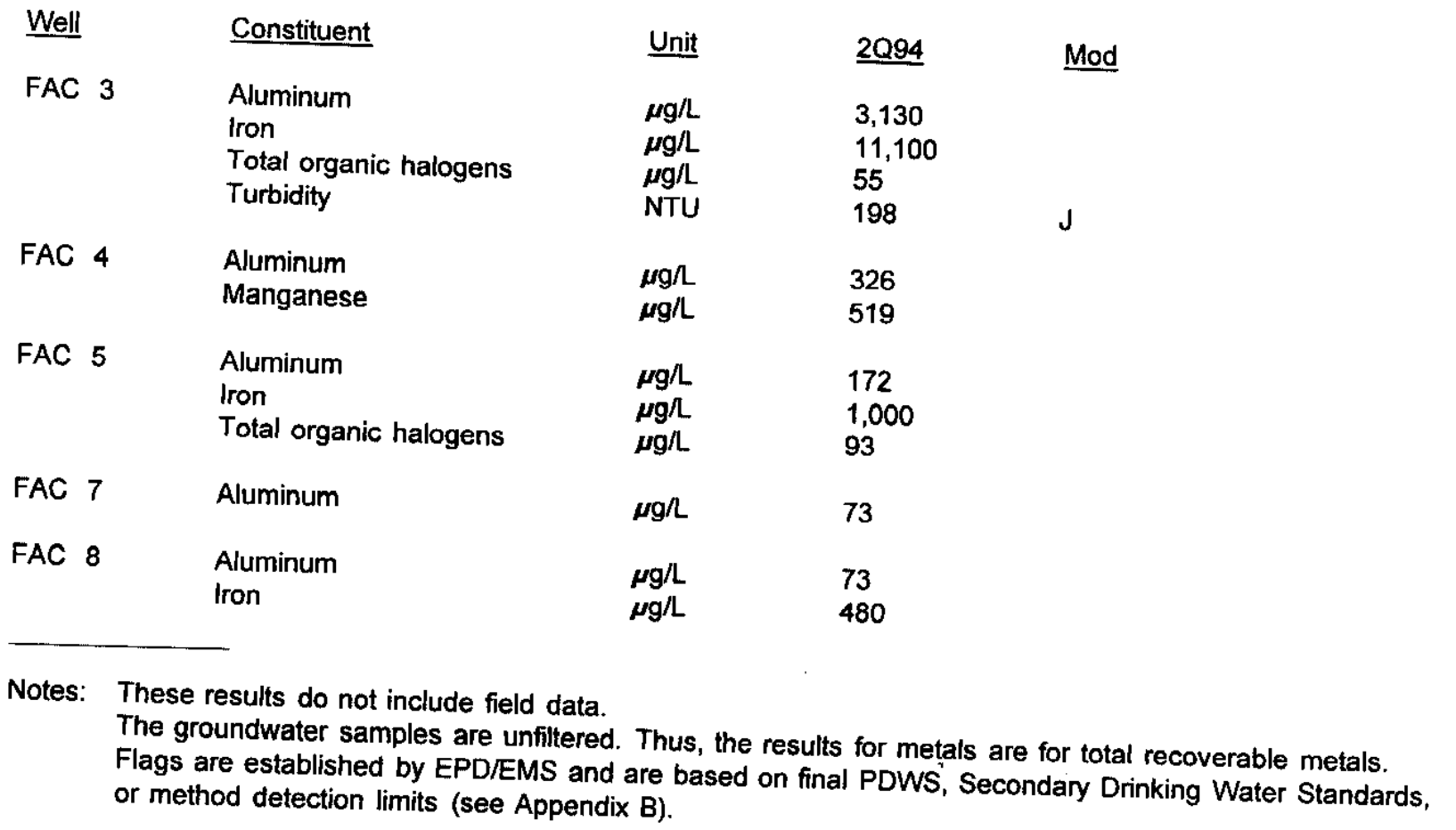




\section{Table 3. Groundwater Monitoring Results for Individual Wells}

\section{WELL FAC 3}

$\begin{array}{lllllll}\text { SRS Coord. } & \text { Lat/Longitude } & \text { Screen Zone Elevation } & \text { Top of Casing } & \text { Casing } & \text { Pump } & \text { Formation } \\ \text { N78018.3 } & \begin{array}{llllll}33.288592^{\circ} \mathrm{N} \\ \text { E55322.7 }\end{array} & \begin{array}{l}81.6726744^{\circ} \mathrm{W} \\ 254.8-224.8 \mathrm{ft} \mathrm{msl}\end{array} & 311.8 \mathrm{ft} \mathrm{msl} & 4^{\prime \prime} \mathrm{PVC} & \text { B } & \text { Water Table }\end{array}$

\section{FIELD MEASUREMENTS}

Sample date: 05/19/94

Depth to water: $81.85 \mathrm{ft}(24.95 \mathrm{~m})$ below TOC

Water elevation: $229.95 \mathrm{ft}(70.09 \mathrm{~m}) \mathrm{ms}$ l

Sp. conductance: $144 \mu \mathrm{S} / \mathrm{cm}$

Turbidity: 457 NTU

Water evacuated before sampling: $5 \mathrm{gal}$

\section{LABORATORY ANALYSES}

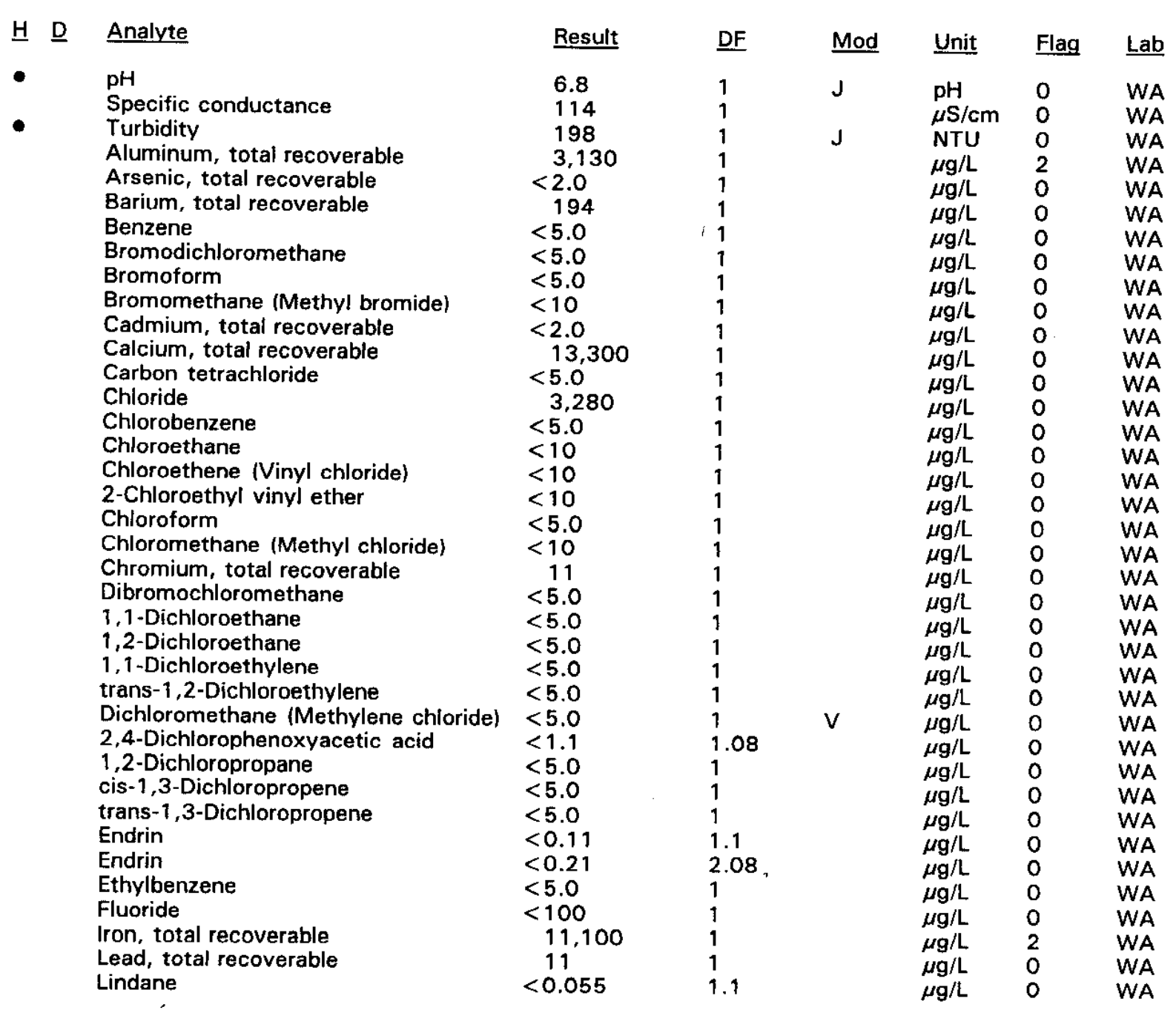

Time: 10:19

pH: 6.6

Alkalinity: $22 \mathrm{mg} / \mathrm{L}$

Water temperature: $19,8^{\circ} \mathrm{C}$

Volumes purged: 1.5 well volumes

- = exceeded holding time. - = exceeded screening level or final primary drinking water standard. 
WELL FAC 3 collected on 05/19/94, laboratory analyses (cont.)

\section{H $\underline{\text { Analyte }}$}

Lindane

Magnesium, total recoverable

Manganese, total recoverable

Mercury, total recoverable

Mercury, total recoverable

Methoxychlor

Methoxychlor

Methoxychlor

Nitrate as nitrogen

Phenols

Potassium, total recoverable

Selenium, total recoverable

Silica, total recoverable

Silver, total recoverable

Sodium, total recoverable

Sulfate

1,1,2,2-Tetrachloroethane

Tetrachloroethylene

Toluene

Total dissolved solids

Total organic carbon

Total organic halogens

Total phosphates (as P)

Toxaphene

Toxaphene

Toxaphene

2,4,5-TP (Silvex)

1,1,1-Trichloroethane

1,1,2-Trichloroethane

Trichloroethylene

Trichlorofluoromethane

- Gross alpha

Nonvolatile beta

Radium-226

Radium-228

Tritium

Tritium

\section{Result}

$<0.10$

2,120

38

0.26

0.27

$<1.0$

$<1.0$

$<0.55$

203

$<5.0$

3.370

$<2.0$

12,700

$<2.0$

3,340

29,700

$<5.0$

$<5.0$

$<5.0$

98,000

2,880

55

141

$<1.1$

$<2.1$

$<2.1$

$<0.54$

$<5.0$

$<5.0$

$<5.0$

$<5.0$

2.3E + 01

$2.5 \mathrm{E}+01$

$4.5 E+00$

1. $2 \mathrm{E}+0 \mathrm{O}$

7.2E-01

6.3E-01
DF $\quad$ Mod 2.08

1

1

2.08

2.08

1.1

1

1

1

1

2.1

1

1

10

1

1
1

1

1

1

1.1

2.08

2.08

1.08

1

1

1

1

1

1

1

1

1

\begin{tabular}{|c|c|c|}
\hline Unit & Flag & $\underline{L a b}$ \\
\hline$\mu \mathrm{g} / \mathrm{L}$ & 0 & WA \\
\hline$\mu \mathrm{g} / \mathrm{L}$ & 0 & WA \\
\hline$\mu \mathrm{g} / \mathrm{L}$ & 1 & WA \\
\hline$\mu \mathrm{g} / \mathrm{L}$ & 0 & WA \\
\hline$\mu \mathrm{g} / \mathrm{L}$ & 0 & WA \\
\hline$\mu \mathrm{g} / \mathrm{L}$ & 0 & WA \\
\hline$\mu \mathrm{g} / \mathrm{L}$ & 0 & WA \\
\hline$\mu \mathrm{g} / \mathrm{L}$ & 0 & WA \\
\hline$\mu \mathrm{g} / \mathrm{L}$ & 0 & WA \\
\hline$\mu \mathrm{g} / \mathrm{L}$ & 0 & WA \\
\hline$\mu \mathrm{g} / \mathrm{L}$ & 0 & WA \\
\hline$\mu \mathrm{g} / \mathrm{L}$ & 0 & WA \\
\hline$\mu g / L$ & 0 & WA \\
\hline$\mu \mathrm{g} / \mathrm{L}$ & 0 & WA \\
\hline$\mu \mathrm{g} / \mathrm{L}$ & 0 & WA \\
\hline$\mu \mathrm{g} / \mathrm{L}$ & 0 & WA \\
\hline$\mu \mathrm{g} / \mathrm{L}$ & 0 & WA \\
\hline$\mu \mathrm{g} / \mathrm{L}$ & 0 & WA \\
\hline$u g / L$ & 0 & WA \\
\hline$h$ & 0 & WA \\
\hline$/ g / L$ & 0 & WA \\
\hline /L & 2 & WA \\
\hline$\pi$ & 0 & WA \\
\hline & 0 & WA \\
\hline & 0 & WA \\
\hline $\mathrm{g} / \mathrm{L}$ & $\begin{array}{l}0 \\
0\end{array}$ & $\begin{array}{l}\text { WA } \\
\text { WA }\end{array}$ \\
\hline$g / L$ & 0 & WA \\
\hline$g / L$ & 0 & WA \\
\hline$g / L$ & 0 & WA \\
\hline$g / L$ & 0 & WA \\
\hline $\mathrm{Ci} / \mathrm{L}$ & 2 & $T M$ \\
\hline $\mathrm{Ci} / \mathrm{L}$ & 0 & TM \\
\hline $\mathrm{Ci} / \mathrm{L}$ & 0 & TM \\
\hline $\mathrm{Ci} / \mathrm{L}$ & 0 & TM \\
\hline $\mathrm{Ci} / \mathrm{m}$ & 0 & TM \\
\hline $\mathrm{C} \mathrm{i} / \mathrm{mL}$ & 0 & TM \\
\hline
\end{tabular}

- = exceeded holding time. $=$ exceeded screening level or final primary drinking water standard. 


\section{WELL FAC 4}

\begin{tabular}{|c|c|c|c|c|c|c|}
\hline SRS Coord. & Lat/Longitude & Screen Zone Elevation & Top of Casing & Casing & Pump & Formation \\
\hline $\begin{array}{l}\text { N78223.8 } \\
\text { E55472.9 }\end{array}$ & $\begin{array}{l}33.289292^{\circ} \mathrm{N} \\
81.672678^{\circ} \mathrm{W}\end{array}$ & $237.8-207.8 \mathrm{ft} \mathrm{ms!}$ & $309.9 \mathrm{ft} \mathrm{msl}$ & 4" PVC & $\mathrm{S}$ & Water Table \\
\hline
\end{tabular}

\section{FIELD MEASUREMENTS}

Sample date: 05/18/94

Depth to water: $80.91 \mathrm{ft}(24.66 \mathrm{~m})$ below TOC Water elevation: $228.99 \mathrm{ft}(69.80 \mathrm{~m}) \mathrm{ms}$

Sp. conductance: $261 \mu \mathrm{S} / \mathrm{cm}$

Turbidity: 0.9 NTU

Water evacuated before sampling: $111 \mathrm{gal}$

\section{LABORATORY ANALYSES}

H $\underline{\text { Analyte }}$

- $\quad$ pH

Specific conductance

- Turbidity

Turbidity

Aluminum, total recoverable

Arsenic, total recoverable

Barium, total recoverable

Benzene

Bromodichloromethane

Bromoform

Bromomethane (Methyl bromide)

Cadmium, total recoverable

Calcium, total recoverable

Carbon tetrachloride

Chloride

Chlorobenzene

Chloroethane

Chloroethene (Vinyl chloride)

2-Chloroethyl vinyl ether

Chloroform

Chloromethane (Methyl chloride)

Chromium, total recoverable

Dibromochloromethane

1,1-Dichloroethane

1,2-Dichloroethane

1,1-Dichloroethylene

trans-1,2-Dichloroethylene

Dichloromethane (Methylene chloride)

2,4-Dichlorophenoxyacetic acid

1,2-Dichloropropane

cis-1,3-Dichloropropene

trans-1,3-Dichloropropene

Endrin

Ethylbenzene

Fluoride

Iron, total recoverable

Lead, total recoverable

Lindane

\begin{tabular}{|c|c|c|c|c|c|}
\hline Result & DF & Mod & Unit & Flag & $\underline{\text { Lab }}$ \\
\hline 5.2 & 1 & $J$ & $\mathrm{pH}$ & 0 & WA \\
\hline 5.3 & 1 & $J$ & $\mathrm{pH}$ & 0 & WA \\
\hline 230 & 1 & & $\mu \mathrm{S} / \mathrm{cm}$ & 0 & WA \\
\hline 0.44 & 1 & $J$ & NTU & 0 & WA \\
\hline 0.44 & 1 & $J$ & NTU & 0 & WA \\
\hline 326 & 1 & & $\mu \mathrm{g} / \mathrm{L}$ & 2 & WA \\
\hline$<2.0$ & 1 & & $\mu \mathrm{g} / \mathrm{L}$ & 0 & WA \\
\hline 63 & 1 & & $\mu \mathrm{g} / \mathrm{L}$ & 0 & WA \\
\hline$<5.0$ & 1 & & $\mu \mathrm{g} / \mathrm{L}$ & 0 & WA \\
\hline$<5.0$ & 1 & & $\mu \mathrm{g} / \mathrm{L}$ & 0 & WA \\
\hline$<5.0$ & 1 & & $\mu \mathrm{g} / \mathrm{L}$ & 0 & WA \\
\hline$<10$ & 1 & & $\mu \mathrm{g} / \mathrm{L}$ & 0 & WA \\
\hline$<2.0$ & 1 & & $\mu \mathrm{g} / \mathrm{L}$ & 0 & WA \\
\hline 11,800 & 1 & & $\mu \mathrm{g} / \mathrm{L}$ & 0 & WA \\
\hline$<5.0$ & 1 & & $\mu \mathrm{g} / \mathrm{L}$ & 0 & WA \\
\hline 4,520 & 1 & & $\mu \mathrm{g} / \mathrm{L}$ & 0 & WA \\
\hline$<5.0$ & 1 & & $\mu g / L$ & 0 & WA \\
\hline$<10$ & 1 & & $\mu \mathrm{g} / \mathrm{L}$ & 0 & WA \\
\hline$<10$ & 1 & & $\mu \mathrm{g} / \mathrm{L}$ & 0 & WA \\
\hline$<10$ & 1 & & $\mu \mathrm{g} / \mathrm{L}$ & 0 & WA \\
\hline$<5.0$ & 1 & & $\mu \mathrm{g} / \mathrm{L}$ & 0 & WA \\
\hline$<10$ & 1 & & $\mu \mathrm{g} / \mathrm{L}$ & 0 & WA \\
\hline$<4.0$ & 1 & & $\mu g / L$ & 0 & WA \\
\hline$<5.0$ & 1 & & $\mu \mathrm{g} / \mathrm{L}$ & 0 & WA \\
\hline$<5.0$ & 1 & & $\mu \mathrm{g} / \mathrm{L}$ & 0 & WA \\
\hline$<5.0$ & 1 & & $\mu \mathrm{g} / \mathrm{L}$ & 0 & WA \\
\hline 5.0 & 1 & & $\mu \mathrm{g} / \mathrm{L}$ & 0 & WA \\
\hline 5.0 & 1 & & $\mu \mathrm{g} / \mathrm{L}$ & 0 & WA \\
\hline$<5.0$ & 1 & V & $\mu \mathrm{g} / \mathrm{L}$ & 0 & WA \\
\hline$<1.1$ & 1.08 & & $\mu g / L$ & 0 & WA \\
\hline 5.0 & 1 & & $\mu g / L$ & 0 & WA \\
\hline 5.0 & 1 & & $\mu \mathrm{g} / \mathrm{L}$ & 0 & WA \\
\hline 5.0 & 1 & & $\mu \mathrm{g} / \mathrm{L}$ & 0 & WA \\
\hline$=0.10$ & 1.04 & & $\mu \mathrm{g} / \mathrm{L}$ & 0 & WA \\
\hline 5.0 & 1 & & $\mu \mathrm{g} / \mathrm{L}$ & 0 & WA \\
\hline 100 & & & $\mu \mathrm{g} / \mathrm{L}$ & 0 & WA \\
\hline 92 & 1 & & $\mu \mathrm{g} / \mathrm{L}$ & 0 & WA \\
\hline$: 3.0$ & 1 & & $\mu \mathrm{g} / \mathrm{L}$ & 0 & WA \\
\hline 0.052 & 1.04 & & $\mu \mathrm{g} / \mathrm{L}$ & 0 & WA \\
\hline
\end{tabular}

Time: 11:54

$\mathrm{pH}: 4.7$

Alkalinity: $0 \mathrm{mg} / \mathrm{L}$

Water temperature: $21.7^{\circ} \mathrm{C}$

Volumes purged: 8.0 well volumes 
WELL FAC 4 collected on 05/18/94, laboratory analyses (cont.)

\section{H D Analyte}

Magnesium, total recoverable Manganese, total recoverable Mercury, total recoverable Methoxychior

Nitrate as nitrogen

Phenols

Phenols

Potassium, total recoverable

Selenium, total recoverable

Silica, total recoverable

Silver, total recoverable

Sodium, total recoverable

Sulfate

1,1,2,2-Tetrachloroethane

Tetrachloroethylene

Toluene

Total dissolved solids

Total organic carbon

Total organic halogens

Total phosphates (as P)

Toxaphene

2,4,5-TP (Silvex)

1,1,1-Trichloroethane

1,1,2-Trichloroethane

Trichloroethylene

Trichlorofluoromethane

Gross alpha

Gross alpha

Nonvolatile beta

Nonvolatile beta

Radium-226

Radium-228

Tritium

Tritium

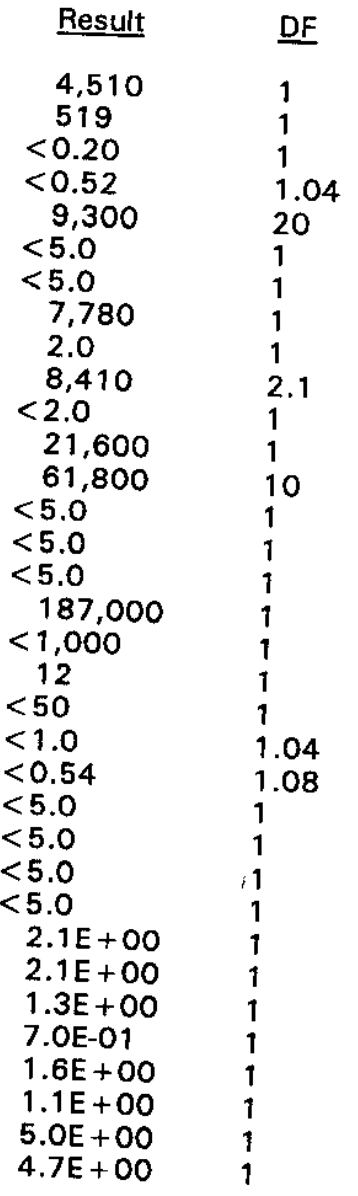

- = exceeded holding time. exceeded screening level or final primary drinking water standard. 
WELL FAC 5

\begin{tabular}{|c|c|c|c|c|c|c|}
\hline SRS Coord. & Lat/Longitude & Screen Zone Elevation & Top of Casing & Casing & Pump & Formation \\
\hline $\begin{array}{l}N 77960.3 \\
\text { E55241.3 }\end{array}$ & $\begin{array}{l}33.288331^{\circ} \mathrm{N} \\
81.672776^{\circ} \mathrm{W}\end{array}$ & $234.0-214.0 \mathrm{ft} \mathrm{msl}$ & $315.8 \mathrm{ft} \mathrm{ms}$ & 4" PVC & $s$ & Wate \\
\hline
\end{tabular}

FIELD MEASUREMENTS

Sample date: 05/19/94

Depth to water: $88.34 \mathrm{ft}(26.93 \mathrm{~m})$ below TOC

Water elevation: $227.46 \mathrm{ft}(69.33 \mathrm{~m}) \mathrm{msl}$

Sp. conductance: $177 \mu \mathrm{S} / \mathrm{cm}$

Turbidity: 9.6 NTU

Water evacuated before sampling: 9 gal

The well went dry during purging.

Time: $8: 59$

pH: 4.8

Alkalinity: $0 \mathrm{mg} / \mathrm{L}$

Water temperature: $18.6^{\circ} \mathrm{C}$

Volumes purged: 1.0 well volumes

\section{LABORATORY ANALYSES}

H D Analyte

- $\quad \mathrm{pH}$

Specific conductance

Turbidity

Aluminum, total recoverable

Arsenic, total recoverable

Barium, total recoverable

Benzene

Bromodichloromethane

Bromoform

Bromomethane (Methyl bromide)

Cadmium, total recoverable

Calcium, total recoverable

Carbon tetrachloride

Chloride

Chlorobenzene

Chloroethane

Chloroethene (Vinyl chloride)

2-Chloroethyl vinyl ether

Chloroform

Chloromethane (Methyl chloride)

Chromium, total recoverable

Dibromochloromethane

1,1-Dichloroethane

1,2-Dichloroethane

1,1-Dichloroethylene

trans-1,2-Dichloroethylene

Dichloromethane (Methylene chloride)

2,4-Dichlorophenoxyacetic acid

2,4-Dichlorophenoxyacetic acid

1,2-Dichloropropane

cis-1,3-Dichloropropene

trans-1,3-Dichloropropene

Endrin

Ethylbenzene

Fluoride

Iron, total recoverabie

Lead, total recoverable

Lindane

Magnesium, total recoverable

\begin{tabular}{|c|c|c|c|c|c|}
\hline Result & $\underline{D F}$ & Mod & Unit & Flag & Lab \\
\hline 5.2 & 1 & $J$ & $\mathrm{pH}$ & 0 & WA \\
\hline 133 & 1 & & $\mu \mathrm{S} / \mathrm{cm}$ & 0 & WA \\
\hline 3.4 & 1 & $J$ & NTU & 0 & WA \\
\hline 172 & 1 & & $\mu \mathrm{g} / \mathrm{L}$ & 2 & WA \\
\hline$<2.0$ & 1 & & $\mu \mathrm{g} / \mathrm{L}$ & 0 & WA \\
\hline 30 & 1 & & $\mu \mathrm{g} / \mathrm{L}$ & 0 & WA \\
\hline$<5.0$ & 1 & & $\mu \mathrm{g} / \mathrm{L}$ & 0 & WA \\
\hline$<5.0$ & 1 & & $\mu g / L$ & 0 & WA \\
\hline$<5.0$ & 1 & & $\mu \mathrm{g} / \mathrm{L}$ & 0 & WA \\
\hline$<10$ & 1 & & $\mu \mathrm{g} / \mathrm{L}$ & 0 & WA \\
\hline$<2.0$ & 1 & & $\mu \mathrm{g} / \mathrm{L}$ & 0 & WA \\
\hline 9,990 & 1 & & $\mu \mathrm{g} / \mathrm{L}$ & 0 & WA \\
\hline$<5.0$ & 1 & & $\mu \mathrm{g} / \mathrm{L}$ & 0 & WA \\
\hline 3,480 & 1 & & $\mu g / L$ & 0 & WA \\
\hline$<5.0$ & 1 & & $\mu \mathrm{g} / \mathrm{L}$ & 0 & WA \\
\hline$<10$ & 1 & & $\mu \mathrm{g} / \mathrm{L}$ & 0 & WA \\
\hline$<10$ & 1 & & $\mu \mathrm{g} / \mathrm{L}$ & 0 & WA \\
\hline$<10$ & 1 & & $\mu g / L$ & 0 & WA \\
\hline$<5.0$ & 1 & & $\mu \mathrm{g} / \mathrm{L}$ & 0 & WA \\
\hline$<10$ & 1 & & $\mu \mathrm{g} / \mathrm{L}$ & 0 & WA \\
\hline$<4.0$ & 1 & & $\mu \mathrm{g} / \mathrm{L}$ & 0 & WA \\
\hline$<5.0$ & 1 & & $\mu \mathrm{g} / \mathrm{L}$ & 0 & WA \\
\hline$<5.0$ & 1 & & $\mu \mathrm{g} / \mathrm{L}$ & 0 & WA \\
\hline$<5.0$ & 1 & & $\mu \mathrm{g} / \mathrm{L}$ & 0 & WA \\
\hline$<5.0$ & 1 & & $\mu \mathrm{g} / \mathrm{L}$ & 0 & WA \\
\hline$<5.0$ & 1 & & $\mu g / L$ & 0 & WA \\
\hline$<5.0$ & 1 & V & $\mu \mathrm{g} / \mathrm{L}$ & 0 & WA \\
\hline$<1.1$ & 1.11 & & $\mu \mathrm{g} / \mathrm{L}$ & 0 & WA \\
\hline$<2.2$ & 2.17 & & $\mu \mathrm{g} / \mathrm{L}$ & 0 & WA \\
\hline$<5.0$ & 1 & & $\mu \mathrm{g} / \mathrm{L}$ & 0 & WA \\
\hline$<5.0$ & 1 & & $\mu \mathrm{g} / \mathrm{L}$ & 0 & WA \\
\hline$<5.0$ & & & $\mu \mathrm{g} / \mathrm{L}$ & 0 & WA \\
\hline$<0.11$ & 1.06 & & $\mu \mathrm{g} / \mathrm{L}$ & 0 & WA \\
\hline$<5.0$ & & & $\mu \mathrm{g} / \mathrm{L}$ & 0 & WA \\
\hline$<100$ & & - & $\mu \mathrm{g} / \mathrm{L}$ & 0 & WA \\
\hline 1,000 & & & $\mu \mathrm{g} / \mathrm{L}$ & 2 & WA \\
\hline 3.7 & 1 & & $\mu \mathrm{g} / \mathrm{L}$ & 0 & WA \\
\hline$<0.053$ & 1.06 & & $\mu \mathrm{g} / \mathrm{L}$ & 0 & WA \\
\hline & 1 & & $\mu \mathrm{g} / \mathrm{L}$ & 0 & WA \\
\hline
\end{tabular}

\footnotetext{
- = exceeded holding time. $\square=$ exceeded screening level or final primary drinking water standard.
} 
WELL FAC 5 collected on 05/19/94, laboratory analyses (cont.)

\section{H $\underline{\text { Analyte }}$}

Manganese, total recoverable

Mercury, total recoverable

Methoxychlor

Nitrate as nitrogen

Phenols

Potassium, total recoverable

Selenium, total recoverable

Silica, total recoverable

Silver, total recoverable

Sodium, total recoverable

Sulfate

1,1,2,2-Tetrachloroethane

Tetrachloroethylene

Toluene

Total dissolved solids

Total organic carbon

Total organic carbon

Total organic halogens

Total phosphates (as P)

Toxaphene

2,4,5-TP (Silvex)

2,4,5-TP (Silvex)

1,1,1-Trichloroethane

1,1,2-Trichloroethane

Trichloroethylene

Trichlorofluoromethane

Gross alpha

Nonvolatile beta

Radium-226

Radium-226

Radium-228

Radium-228

Tritium

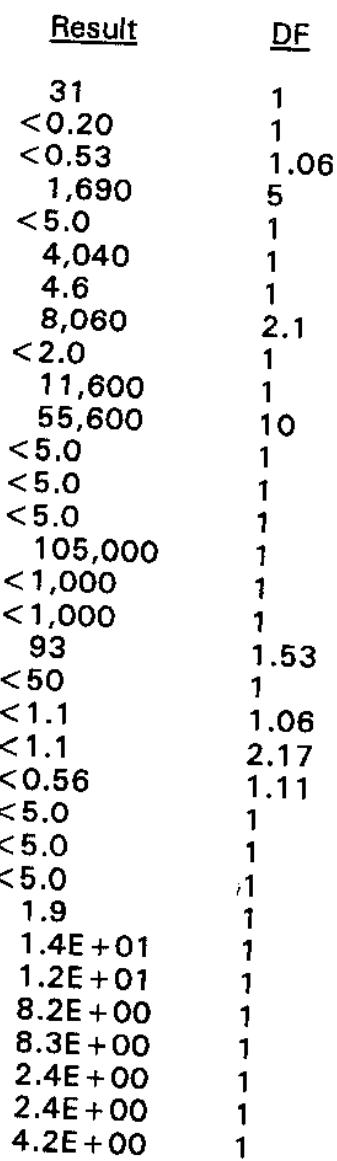

\section{WELL FAC 5P}

$\begin{array}{cccc}\text { Unit } & & \text { Flag } & \text { Lab } \\ \mu \mathrm{g} / \mathrm{L} & 1 & \text { WA } \\ \mu \mathrm{g} / \mathrm{L} & 0 & \text { WA } \\ \mu \mathrm{g} / \mathrm{L} & 0 & \text { WA } \\ \mu \mathrm{g} / \mathrm{L} & 0 & \text { WA } \\ \mu \mathrm{g} / \mathrm{L} & 0 & \text { WA } \\ \mu \mathrm{g} / \mathrm{L} & 0 & \text { WA } \\ \mu \mathrm{g} / \mathrm{L} & 0 & \text { WA } \\ \mu \mathrm{g} / \mathrm{L} & 0 & \text { WA } \\ \mu \mathrm{g} / \mathrm{L} & 0 & \text { WA } \\ \mu \mathrm{g} / \mathrm{L} & 0 & \text { WA } \\ \mu \mathrm{g} / \mathrm{L} & 0 & \text { WA } \\ \mu \mathrm{g} / \mathrm{L} & 0 & \text { WA } \\ \mu \mathrm{g} / \mathrm{L} & 0 & \text { WA } \\ \mu \mathrm{g} / \mathrm{L} & 0 & \text { WA } \\ \mu \mathrm{g} / \mathrm{L} & 0 & \text { WA } \\ \mu \mathrm{g} / \mathrm{L} & 0 & \text { WA } \\ \mu \mathrm{g} / \mathrm{L} & 0 & \text { WA } \\ \mu \mathrm{g} / \mathrm{L} & 2 & \text { WA } \\ \mu \mathrm{g} / \mathrm{L} & 0 & \text { WA } \\ \mu \mathrm{g} / \mathrm{L} & 0 & \text { WA } \\ \mu \mathrm{g} / \mathrm{L} & 0 & \text { WA } \\ \mu \mathrm{g} / \mathrm{L} & 0 & \text { WA } \\ \mu \mathrm{g} / \mathrm{L} & 0 & \text { WA } \\ \mu \mathrm{g} / \mathrm{L} & 0 & \text { WA } \\ \mu \mathrm{g} / \mathrm{L} & 0 & \text { WA } \\ \mu \mathrm{g} / \mathrm{L} & 0 & \text { WA } \\ \mathrm{pC \textrm {i } / \mathrm { L }} & 1 & \text { TM } \\ \mathrm{pCi} / \mathrm{L} & 0 & \text { TM } \\ \rho \mathrm{Ci} / \mathrm{L} & 0 & \text { TM } \\ \mathrm{\rho C \textrm {i }} / \mathrm{L} & 0 & \text { TM } \\ \mathrm{\rho Ci} / \mathrm{L} & 0 & \text { TM } \\ \mathrm{pCi} / \mathrm{L} & 0 & \text { TM } \\ \mathrm{\rho Ci} / \mathrm{mL} & 0 & \text { TM } \\ & & 0 & \end{array}$

\begin{tabular}{|c|c|c|c|c|c|c|}
\hline is Coord. & Lat/Longitude & Screen Zone Elevation & Top of Casing & Casing & Pump & \\
\hline 53 & $\begin{array}{l}38927^{\circ} \mathrm{N} \\
3001^{\circ} \mathrm{W}\end{array}$ & $235.7-225.7 \mathrm{ft} \mathrm{msl}$ & $313 \mathrm{ft} \mathrm{msl}$ & 2" PVC & & Water Tabie \\
\hline
\end{tabular}

FIELD MEASUREMENTS

Sample date: 05/18/94

Depth to water: $83.91 \mathrm{ft}(25.58 \mathrm{~m})$ below TOC

Time: 10:59

Water elevation: $229.09 \mathrm{ft}(69.83 \mathrm{~m}) \mathrm{msl}$

No water evacuated before sampling.

The well pumped dry before all field parameters were collected.

\footnotetext{
- = exceeded holding time. $\bullet=$ exceeded screening level or final primary drinking water standard.
} 


\section{WELL FAC 6}

\begin{tabular}{|c|c|c|c|c|c|c|}
\hline SRS Coord. & Lat/Longitude & Screen Zone Elevation & Top of Casing & $\underline{\text { Casing }}$ & Pump & Formation \\
\hline $\begin{array}{l}781 \\
653\end{array}$ & $\begin{array}{l}33.288858^{\circ} \mathrm{N} \\
81.672855^{\circ} \mathrm{W}\end{array}$ & $236.2-216.2 \mathrm{ft} \mathrm{msl}$ & $312.5 \mathrm{ft} \mathrm{msl}$ & 4" PVC & $\mathbf{s}$ & \\
\hline
\end{tabular}

\section{FIELD MEASUREMENTS}

Sample date: 05/18/94

Depth to water: $90.94 \mathrm{ft}(27.72 \mathrm{~m})$ below TOC

Time: $11: 10$

Water elevation: $221.56 \mathrm{ft}(67.53 \mathrm{~m}) \mathrm{msl}$

No water evacuated before sampling.

The well pumped dry before all field parameters were collected.

\section{WELL FAC 7}

\begin{tabular}{|c|c|c|c|c|c|c|}
\hline & Lat/Longitude & Screen Zone Elevation & Top of Casing & Casing & Pump & \\
\hline $\begin{array}{l}N 781 \\
=553\end{array}$ & $\begin{array}{l}33.288879^{\circ} \mathrm{N} \\
81.672790^{\circ} \mathrm{W}\end{array}$ & $0050-70$ & $312 \mathrm{ft} \mathrm{msl}$ & 4" P & $s$ & Water Table \\
\hline
\end{tabular}

\section{FIELD MEASUREMENTS}

Sample date: 05/19/94

Depth to water: $83.79 \mathrm{ft}(25.54 \mathrm{~m})$ below TOC

Water elevation: $228.21 \mathrm{ft}(69.56 \mathrm{~m}) \mathrm{msl}$

Sp. conductance: $35 \mu \mathrm{S} / \mathrm{cm}$

Turbidity: 12.3 NTU

Water evacuated before sampling: $5 \mathrm{gal}$

The well went dry during purging.

\section{LABORATORY ANALYSES}

H $\underline{\text { Analyte }}$

- $\mathrm{pH}$

Specific conductance

Turbidity

Aluminum, total recoverable

Arsenic, total recoverable

Barium, total recoverable

Benzene

Bromodichloromethane

Bromoform

Bromomethane (Methyl bromide)

Cadmium, total recoverable

Calcium, total recoverable

Carbon tetrachloride

Chloride

Chlorobenzene

Chloroethane

Chloroethene (Vinyl chloride)

2-Chloroethyl vinyl ether

Chloroform

Chloromethane (Methyl chloride)

Chromium, total recoverable

Dibromochloromethane
Time: $8: 37$

pH: 5.4

Alkalinity: $1 \mathrm{mg} / \mathrm{L}$

Water temperature: $18.9^{\circ} \mathrm{C}$

Volumes purged: 0.6 well volumes

$\begin{array}{llllll}\text { Result } & \text { DF } & \text { Mod } & \underline{\text { Unit }} & \text { Flag } & \text { Lab } \\ 5.5 & 1 & & & & \\ 24 & 1 & & \mu \mathrm{pH} & 0 & \text { WA } \\ 3.0 & 1 & \mathrm{~J} & \mu \mathrm{NT} / \mathrm{cm} & 0 & \text { WA } \\ 73 & 1 & & \mu \mathrm{g} / \mathrm{L} & 2 & \text { WA } \\ <2.0 & 1 & & \mu \mathrm{g} / \mathrm{L} & 0 & \text { WA } \\ <4.0 & 1 & & \mu \mathrm{g} / \mathrm{L} & 0 & \text { WA } \\ <5.0 & 1 & & \mu \mathrm{g} / \mathrm{L} & 0 & \text { WA } \\ <5.0 & 1 & & \mu \mathrm{g} / \mathrm{L} & 0 & \text { WA } \\ <5.0 & 1 & & \mu \mathrm{g} / \mathrm{L} & 0 & \text { WA } \\ <10 & 1 & & \mu \mathrm{g} / \mathrm{L} & 0 & \text { WA } \\ <2.0 & 1 & & \mu \mathrm{g} / \mathrm{L} & 0 & \text { WA } \\ 338 & 1 & & \mu \mathrm{g} / \mathrm{L} & 0 & \text { WA } \\ <5.0 & 1 & & \mu \mathrm{g} / \mathrm{L} & 0 & \text { WA } \\ 2.800 & 1 & & \mu \mathrm{g} / \mathrm{L} & 0 & \text { WA } \\ <5.0 & 1 & & \mu \mathrm{g} / \mathrm{L} & 0 & \text { WA } \\ <10 & 1 & & \mu \mathrm{g} / \mathrm{L} & 0 & \text { WA } \\ <10 & 1 & & \mu \mathrm{g} / \mathrm{L} & 0 & \text { WA } \\ <10 & 1 & & \mu \mathrm{g} / \mathrm{L} & 0 & \text { WA } \\ <5.0 & 1 & & \mu \mathrm{g} / \mathrm{L} & 0 & \text { WA } \\ <10 & 1 & & \mu \mathrm{g} / \mathrm{L} & 0 & \text { WA } \\ <4.0 & 1 & & \mu \mathrm{g} / \mathrm{L} & 0 & \text { WA } \\ <5.0 & 1 & & \mu \mathrm{g} / \mathrm{L} & 0 & \text { WA }\end{array}$

\footnotetext{
- = exceeded holding time. - = exceeded screening level or final primary drinking water standard.
} 
WELL FAC 7 collected on 05/19/94, laboratory analyses (cont.)

H $\quad$ Analyte

1,1-Dichloroethane

1,2-Dichloroethane

1,1-Dichloroethylene

trans-1,2-Dichloroethylene

Dichloromethane (Methylene chloride)

2,4-Dichlorophenoxyacetic acid

1,2-Dichloropropane

cis-1,3-Dichloropropene

trans-1,3-Dichloropropene
Endrin

Ethylbenzene

Fluoride

Iron, total recoverable

Lead, total recoverable

Lindane

Magnesium, total recoverable

Manganese, total recoverable

Mercury, total recoverable

Methoxychlor

Nitrate as nitrogen

Phenols

Potassium, total recoverable

Selenium, total recoverable

Silica, total recoverable

Silver, total recoverable

Sodium, total recoverable

Sulfate

1,1,2,2-Tetrachloroethane

Tetrachloroethylene

Toluene

Total dissolved solids

Total organic carbon

Total organic halogens

Total phosphates (as P)

Toxaphene

2,4,5-TP (Silvex)

1,1,1-Trichloroethane

1,1,2-Trichloroethane

Trichloroethylene

Trichlorofluoromethane

Gross alpha

Nonvolatile beta

Radium-226

Radium-228

Tritium
Result

$<5.0$

$<5.0$

$<5.0$

$<5.0$

$<14$

$<1.1$

$<5.0$

$<5.0$

$<5.0$

$<0.11$

$<5.0$

$<100$

225

4.4

$<0.054$

228

7.4

0.34

$<0.54$

214

$<5.0$

608

$<2.0$

8,430

$<2.0$

4,120

4,430

$<5.0$

$<5.0$

$<5.0$

26,000

$<1,000$

7.8

$<50$

$<1.1$

$<0.55$

$<5.0$

$<5.0$

$<5.0$

$<5.0$

1.7E + 00

$5.6 E+00$

4.0E-O1

$<5.0 E-02$

$2.0 \mathrm{E}+00$
$\underline{D F}$

1

1

1

1

1.1

1

1

1.08

1

1

1

1.08

1

1.08

1

1

1

2.1

1

1

1

1

1

1.08

1.1

1

1

1

1

18

Mod Unit Flag Lab

$\begin{array}{lll}\mu \mathrm{g} / \mathrm{L} & 0 & \text { WA } \\ \mu \mathrm{g} / \mathrm{L} & 0 & \text { WA } \\ \mu \mathrm{g} / \mathrm{L} & 0 & \text { WA }\end{array}$

JV $\quad \mu g / L \quad O \quad W A$

$J V \mu g / L \quad O \quad W A$

$\mu \mathrm{g} / \mathrm{L} \quad 0 \quad$ WA

$\mu g / L \quad 0 \quad W A$

$\mu \mathrm{g} / \mathrm{L} \quad 0$ WA

$\mu g / L \quad 0 \quad W A$

$\mu \mathrm{g} / \mathrm{L} \quad 0 \quad W A$

$\mu \mathrm{g} / \mathrm{L} \quad 0 \quad$ WA

$\mu g / L \quad 0 \quad W A$

$\mu \mathrm{g} / \mathrm{L} \quad 1 \quad$ WA

$\mu \mathrm{g} / \mathrm{L} \quad 0 \quad$ WA

$\mu g / L \quad 0 \quad W A$

$\mu \mathrm{g} / \mathrm{L} \quad 0 \quad$ WA

$\mu \mathrm{g} / \mathrm{L} \quad 0 \quad W A$

$\mu \mathrm{g} / \mathrm{L} \quad 0 \quad W A$

$\mu \mathrm{g} / \mathrm{L} \quad 0 \quad$ WA

$\mu g / L \quad 0 \quad W A$

$\mu \mathrm{g} / \mathrm{L} \quad 0 \quad W A$

$\mu g / L \quad 0 \quad W A$

$\mu \mathrm{g} / \mathrm{L} \quad 0 \quad W A$

$\mu \mathrm{g} / \mathrm{L} \quad 0 \quad$ WA

$\mu \mathrm{g} / \mathrm{L} \quad 0 \quad W A$

$\mu \mathrm{g} / \mathrm{L} \quad 0 \quad W A$

$\mu \mathrm{g} / \mathrm{L} \quad 0 \quad$ WA

$\mu \mathrm{g} / \mathrm{L} \quad 0 \quad W A$

$\mu g / L \quad 0 \quad W A$

$\mu \mathrm{g} / \mathrm{L} \quad 0 \quad W A$

$\mu \mathrm{g} / \mathrm{L} \quad 0 \quad W A$

$\mu g / L \quad 0 \quad W A$

$\mu \mathrm{g} / \mathrm{L} \quad 0 \quad W A$

$\mu \mathrm{g} / \mathrm{L} \quad 0 \quad W A$

$\mu g / L \quad 0 \quad W A$

$\mu \mathrm{g} / \mathrm{L} \quad 0 \quad$ WA

$\mu \mathrm{g} / \mathrm{L} \quad 0 \quad$ WA

$\mu g / L \quad 0 \quad$ WA

$\mu \mathrm{g} / \mathrm{L} \quad \mathrm{O}$ WA

$\mu \mathrm{g} / \mathrm{L} \quad 0 \quad W A$

$\mathrm{pCi} / \mathrm{L} \quad 0 \quad \mathrm{TM}$

pCi/L 00 TM

$p C i / L \quad 0 \quad T M$

pCi/L 00 TM

$\mathrm{pCi} / \mathrm{mL} \quad \mathrm{O} \quad \mathrm{TM}$

- = exceeded holding time. $\square=$ exceeded screening level or final primary drinking water standard. 


\section{WELL FAC 8}

\begin{tabular}{|c|c|c|c|c|c|c|}
\hline SRS Coord. & Lat/Lonqitude & Screen Zone Elevation & Top of Casing & $\underline{\text { Casing }}$ & Pump & Formation \\
\hline $\begin{array}{l}90.9 \\
56.0\end{array}$ & $\begin{array}{l}33.288823^{\circ} \mathrm{N} \\
81.672701^{\circ} \mathrm{W}\end{array}$ & $236.0-216.0 \mathrm{ft} \mathrm{msl}$ & $311 \mathrm{ft} \mathrm{msl}$ & 4" PVC & $\mathrm{s}$ & \\
\hline
\end{tabular}

\section{FIELD MEASUREMENTS}

Sample date: $05 / 19 / 94$

Depth to water: $81.23 \mathrm{ft}\{24.76 \mathrm{~m}\rangle$ below TOC

Water elevation: $229.77 \mathrm{ft}(70.03 \mathrm{~m}) \mathrm{msl}$

Sp. conductance: $55 \mu \mathrm{S} / \mathrm{cm}$

Turbidity: 4.5 NTU

Water evacuated before sampling: $3 \mathrm{gal}$

The well went dry during purging.

\section{LABORATORY ANALYSES}

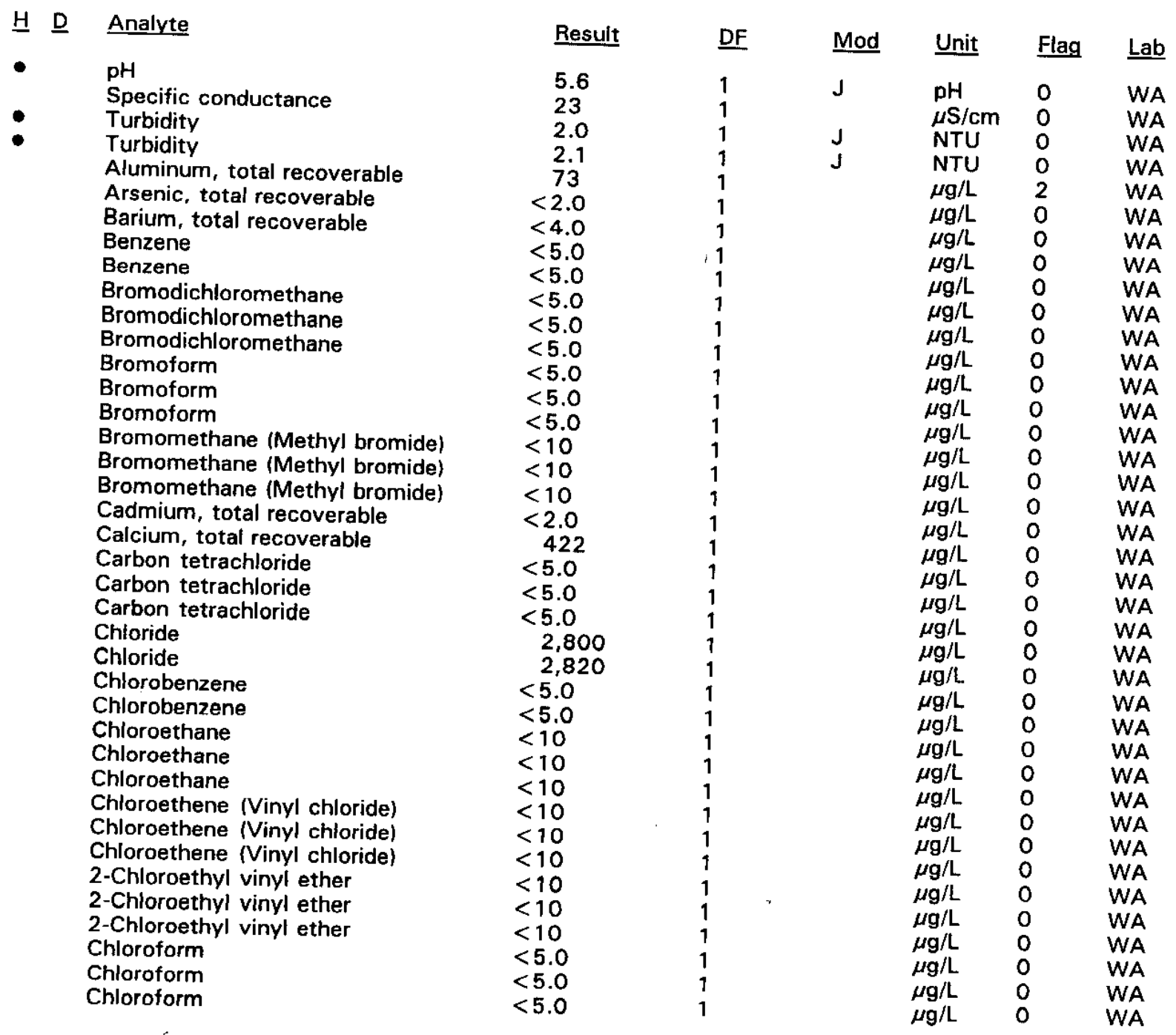

- = exceeded holding time. $\square=$ exceeded screening level or final primary drinking water standard.

Time: $8: 17$

$\mathrm{pH}: 5.4$

Alkalinity: $5 \mathrm{mg} / \mathrm{L}$

Water temperature: $18.6{ }^{\circ} \mathrm{C}$

Volumes purged: 0.3 well volumes 
WELL FAC 8 collected on 05/19/94, laboratory analyses (cont.)

브 $\underline{\text { Analyte }}$

Chloromethane (Methyl chloride)

Chloromethane (Methyl chloride)

Chloromethane (Methyl chloride)

Chromium, total recoverable

Dibromochloromethane

Dibromochloromethane

Dibromochioromethane

1,1-Dichloroethane

1,1-Dichloroethane

1,1-Dichloroethane

1,2-Dichloroethane

1,2-Dichloroethane

1,2-Dichloroethane

1, 1-Dichloroethylene

1,1-Dichloroethylene

trans-1,2-Dichloroethylene

trans-1,2-Dichloroethylene

trans-1,2-Dichloroethylene

Dichloromethane (Methylene chloride)

Dichloromethane (Methylene chloride)

Dichloromethane (Methylene chloride)

2,4-Dichlorophenoxyacetic acid

1,2-Dichloropropane

1,2-Dichloropropane

1,2-Dichloropropane

cis-1,3-Dichloropropene

cis-1,3-Dichloropropene

cis-1,3-Dichloropropene

trans-1,3-Dichloropropene

trans-1,3-Dichloropropene

trans-1,3-Dichloropropene

Endrin

Ethylbenzene

Ethylbenzene

Ethylbenzene

Fluoride

Iron, total recoverable

Lead, total recoverable

Lindane

Magnesium, total recoverable

Manganese, total recoverable

Mercury, total recoverable

Methoxychlor

Nitrate as nitrogen

Phenols

Potassium, total recoverable

Selenium, total recoverable

Silica, total recoverable

Silver, total recoverable

Sodium, total recoverable

Sulfate

1, 1,2,2-Tetrachloroethane

$1,1,2,2$-Tetrachloroethane

1,1,2,2-Tetrachloroethane

Tetrachloroethylene

Tetrachloroethylene
Result

$<10$

$<10$

$<10$

$<4.0$

$<5.0$

$<5.0$

$<5.0$

$<5.0$

$<5.0$

$<5.0$

$<5.0$

$<5.0$

$<5.0$

$<5.0$

$<5.0$

$<5.0$

$<5.0$

$<5.0$

$<5.0$

$<5.0$

$<14$

$<1.1$

$<5.0$

$<5.0$

$<5.0$

$<5.0$

$<5.0$

$<5.0$

$<5.0$

$<5.0$

$<5.0$

$<0.11$

$<5.0$

$<5.0$

$<5.0$

$<100$

480

4.5

$<0.053$

173

5.4

0.36

$<0.53$

169

$<5.0$

675

$<2.0$

8,100

$<2.0$

4,150

3,470

$<5.0$

$<5.0$

$<5.0$

$<5.0$

$<5.0$
DF

Mod

Unit Flag Lab

$\mu \mathrm{g} / \mathrm{L} \quad \mathrm{O} \quad$ WA

$\mu \mathrm{g} / \mathrm{L} \quad \mathrm{W}$ WA

$\mu \mathrm{g} / \mathrm{L} \quad \mathrm{O} \quad$ WA

$\mu \mathrm{g} / \mathrm{L} \quad \mathrm{O}$ WA

$\mu g / L \quad 0 \quad$ WA

$\mu g / L \quad 0 \quad$ WA

$\mu \mathrm{g} / \mathrm{L} \quad 0 \quad$ WA

$\mu g / L \quad 0 \quad$ WA

$\mu \mathrm{g} / \mathrm{L} \quad 0 \quad$ WA

$\mu g / L \quad 0 \quad$ WA

$\mu g / L \quad 0 \quad$ WA

$\mu g / L \quad 0 \quad W A$

$\mu g / L \quad 0 \quad$ WA

$\mu \mathrm{g} / \mathrm{L} \quad 0 \quad$ WA

$\mu g / L \quad 0 \quad$ WA

$\mu \mathrm{g} / \mathrm{L} \quad \mathrm{O} \quad$ WA

$\mu \mathrm{g} / \mathrm{L} \quad 0 \quad$ WA

$\mu \mathrm{g} / \mathrm{L} \quad 0 \quad$ WA

V

$\mu \mathrm{g} / \mathrm{L}$

$\mu \mathrm{g} / \mathrm{L}$

JV

$\mu g / L$

$\mu \mathrm{g} / \mathrm{L}$

$\mu \mathrm{g} / \mathrm{L}$

$\mu \mathrm{g} / \mathrm{L}$

$\mu \mathrm{g} / \mathrm{L}$

$\mu \mathrm{g} / \mathrm{L}$

$\mu \mathrm{g} / \mathrm{L}$

$\mu \mathrm{g} / \mathrm{L}$

$\mu \mathrm{g} / \mathrm{L}$

$\mu g / L$

$\mu \mathrm{g} / \mathrm{L}$

$\mu \mathrm{g} / \mathrm{L}$

$\mu g / L$

$\mu \mathrm{g} / \mathrm{L}$

$\mu g / L$

$\mu \mathrm{g} / \mathrm{L}$

$\mu \mathrm{g} / \mathrm{L}$

$\mu \mathrm{g} / \mathrm{L}$

$\mu \mathrm{g} / \mathrm{L}$

$\mu \mathrm{g} / \mathrm{L}$

$\mu \mathrm{g} / \mathrm{L}$

$\mu \mathrm{g} / \mathrm{L}$

$\mu \mathrm{g} / \mathrm{L}$

$\mu \mathrm{g} / \mathrm{L}$

$\mu \mathrm{g} / \mathrm{L}$

$\mu \mathrm{g} / \mathrm{L}$

$\mu \mathrm{g} / \mathrm{L}$

$\mu \mathrm{g} / \mathrm{L}$

$\mu \mathrm{g} / \mathrm{L}$

$\mu \mathrm{g} / \mathrm{L}$

$\mu \mathrm{g} / \mathrm{L}$

$\mu \mathrm{g} / \mathrm{L}$

$\mu \mathrm{g} / \mathrm{L}$

$\mu \mathrm{g} / \mathrm{L}$

$\mu \mathrm{g} / \mathrm{L}$
WA

WA

WA

WA

WA

WA

WA

WA

WA

WA

WA

WA

WA

WA

WA

WA

WA

WA

WA

WA

WA

WA

WA

WA

WA

WA

WA

WA

WA

WA

WA

WA

WA

WA

WA

WA

WA

WA

- = exceeded holding time.

= exceeded screening level or final primary drinking water standard. 
WELL FAC 8 collected on 05/19/94, laboratory analyses (cont.)

\section{H $\underline{\text { D }}$ Analyte}

Tetrachloroethylene

Toluene

Toluene

Total dissolved solids

Total organic carbon

Total organic halogens

Total phosphates (as P)

Toxaphene

2,4,5-TP (Silvex)

$1,1,1$-Trichloroethane

1,1,1-Trichloroethane

1,1,1-Trichloroethane

1,1,2-Trichloroethane

1,1,2-Trichloroethane

1,1,2-Trichloroethane

Trichloroethylene

Trichloroethylene

Trichlorofluoromethane

Trichlorofluoromethane

Trichlorofluoromethane

Gross alpha

Nonvolatile beta

Radium-226

Radium-228

Tritium

\begin{tabular}{|c|c|}
\hline Result & $\underline{D F}$ \\
\hline$<5.0$ & 1 \\
\hline$<5.0$ & 1 \\
\hline$<5.0$ & 1 \\
\hline 32,000 & 1 \\
\hline$<1,000$ & 1 \\
\hline 7.5 & 1 \\
\hline $\begin{array}{l}<50 \\
<10\end{array}$ & 1 \\
\hline $\begin{array}{l}<1.0 \\
<0.55\end{array}$ & $\begin{array}{l}1.05 \\
1.09\end{array}$ \\
\hline$<5.0$ & 1 \\
\hline$<5.0$ & 1 \\
\hline$<5.0$ & 1 \\
\hline$<5.0$ & 1 \\
\hline$<5.0$ & 1 \\
\hline$<5.0$ & 1 \\
\hline$<5.0$ & 1 \\
\hline$<5.0$ & 1 \\
\hline$<5.0$ & 1 \\
\hline$<5.0$ & 1 \\
\hline$<5.0$ & 1 \\
\hline $4.5 E+00$ & 1 \\
\hline $3.9 E+00$ & 1 \\
\hline $1.4 \mathrm{E}+00$ & 1 \\
\hline $1.6 \mathrm{E}+00$ & 1 \\
\hline $1.9 E+00$ & 1 \\
\hline
\end{tabular}

\begin{tabular}{llll} 
Mod & Unit & Flag & Lab \\
\cline { 2 - 3 }$\mu \mathrm{g} / \mathrm{L}$ & 0 & WA \\
$\mu \mathrm{g} / \mathrm{L}$ & 0 & WA \\
$\mu \mathrm{g} / \mathrm{L}$ & 0 & WA \\
$\mu \mathrm{g} / \mathrm{L}$ & 0 & WA \\
$\mu \mathrm{g} / \mathrm{L}$ & 0 & WA \\
$\mu \mathrm{g} / \mathrm{L}$ & 0 & WA \\
$\mu \mathrm{g} / \mathrm{L}$ & 0 & WA \\
$\mu \mathrm{g} / \mathrm{L}$ & 0 & WA \\
$\mu \mathrm{g} / \mathrm{L}$ & 0 & WA \\
$\mu \mathrm{g} / \mathrm{L}$ & 0 & WA \\
$\mu \mathrm{g} / \mathrm{L}$ & 0 & WA \\
$\mu \mathrm{g} / \mathrm{L}$ & 0 & WA \\
$\mu \mathrm{g} / \mathrm{L}$ & 0 & WA \\
$\mu \mathrm{g} / \mathrm{L}$ & 0 & WA \\
$\mu \mathrm{g} / \mathrm{L}$ & 0 & WA \\
$\mu \mathrm{g} / \mathrm{L}$ & 0 & WA \\
$\mu \mathrm{g} / \mathrm{L}$ & 0 & WA \\
$\mu \mathrm{g} / \mathrm{L}$ & 0 & WA \\
$\mu \mathrm{g} / \mathrm{L}$ & 0 & WA \\
$\mu \mathrm{g} / \mathrm{L}$ & 0 & WA \\
$\mathrm{pCi} / \mathrm{L}$ & 0 & TM \\
$\mathrm{pCi} / \mathrm{L}$ & 0 & TM \\
$\mathrm{pCi} / \mathrm{L}$ & 0 & TM \\
$\mathrm{pCi} / \mathrm{L}$ & 0 & TM \\
$\mathrm{pCi} / \mathrm{mL}$ & 0 & TM \\
& &
\end{tabular}

- = exceeded holding time. $\square=$ exceeded screening level or final primary drinking water standard. 
WSRC-TR-94-0347

Unclassified

THIS PAGE LEFT BLANK INTENTIONALLY. 


\section{Appendix E}

\section{Data Quality/Useability Assessment}


THIS PAGE LEFT BLANK INTÉNTIONALLY. 


\section{Data Quality/Useability Assessment}

Quality assurance/quality control (QAVC) procedures relating to accuracy and precision of analyses performed on groundwater samples are followed in the field and laboratory and are reviewed prior to publication of results. The review by the Environmental Protection Department/ quarter and Monitoring Section (EPD/EMS) of the volume of analytical data acquired each cannot be compled in various reports is an ongoing process; its review of the QAVC data servation and Recover obtain further information and associated regulations. Other site and regulatory personnel can described below.

\section{Data Qualification}

The contract laboratories continually assess their own accuracy and precision according to U.S. Environmental Protection Agency (EPA) guidelines. They submit sample- or batch-specific QAVQ information either at the same time as analytical results or in quarterly summaries. Properly defined and used result modifiers (also referred to as qualifiers) can be a key component in assessing data useability. Result modifiers designed by EPD/EMS and used by the primary laboratories are presented in Appendix D.

\section{Assessment of Accuracy of the Data} Accuracy, or the nearness of the reported result to the true concentration of a constituent in a
sample, can be assessed in several ways.

A laboratory's general accuracy can be judged by analysis of results obtained from known samples. The non-radionuclide contract laboratories analyze commercial reference samples every quarter at EPD/EMS' request. The results of these analyses are presented in the EPD/ EMS groundwater monitoring quarterly reports. The primary laboratories also seek or maintain state certification by participating periodically in performance studies; reference samples and analysis of results are provided by EPA. Results of these studies also are published in the
EPD/EMS quarterly reports.

Analysis of blanks provides a tool for assessing the accuracy of both sampling and laboratory analysis. Results for all field blanks for the quarter can be found in the EPD/EMS quarterly reports. Any field or laboratory blanks that exceed established minimums are identified in the same reports, in tables associating them with groundwater samples analyzed in the same
batches.

Surrogates, organic compounds similar in chemical behavior to the compounds of interest but not normally found in environmental samples, are used to monitor the effect of the matrix on the accuracy of analyses for organic parameters. For example, for analyses of volatile organics by EPA Method 8240, three surrogate compounds are added to all samples and blanks in each analytical batch. In analyses of semivolatile organics, three acid compounds and three base/neutral compounds are used. Two surrogates are used in organochlorine pesticides analyses. Percent recoveries for surrogate analyses are calculated by laboratory personnel, reported to EPD/EMS, reviewed, and entered into the database, but they are not published. If recoveries are not within specified limits, the laboratory is expected to reanalyze the samples or attach qualifiers to the
data identifying the anomalous results. 
Sample-specific accuracy for both organic and inorganic parameters can be assessed by examia baseline set of values. A spike duplicate results. A sample is analyzed unspiked to determine compounds appropriate to the analysesion of the sample is spiked with known concentrations of pounds for volatile organics analyses, eing performed, typically five volatile organic comcide compounds for pesticides, all metals for semivolatile compounds for semivolatiles, six pestiand a known quantity of cyanide for cyanide metals analyses by SW-846 methods (EPA, 1986), that is recovered (i.e., measured in excess analysis. The percentage of the spike compound direct measure of analytical accuracy. EPA the value obtained for the unspiked sample) is a run at least once per 20 samples of similar matrix. Matrix spike/matrix spike duplicate results are reported to EPD/EMS but are not published.
For organic compounds, according to EPA guidelines, no action is spike/matrix spike duplicate data alone (i.e., no result modifion is taken on the basis of matrix modifiers are assigned solely on the basis problem in the analysis of one or more analytes.

In the case of inorganic compounds, such as metals, the matrix spike sample analysis provides information about the effect of each sample matrix on the digestion and measurement methodology. Data qualifiers assigned by the laboratories on the basis of the percentage of spike recovery are reported in the published results tables.

\section{Assessment of Precision}

Precision of the analyses, or agreement of a set of replicate results among themselves, is assessed through the use of duplicates initiated by the laboratory and blind replicates (provided by EPD/EMS). The results of duplicate and replicate analyses are presented in the results tables results are not presented in fourth-quarter reports; the results year. Duplicate and replicate highest result for each analyte for each quarter of the year.

The laboratories assess precision by calculating the relative percent difference, or RPD, for each pair of laboratory-initiated duplicate results. One of the contract laboratories uses a data qualifier (J3) to modify metals analyses when the RPD for laboratory duplicates is greater than $20 \%$.

Additional statistical comparisons of laboratory duplicate and blind replicate results, both intraand interlaboratory, are presented in the EPD/EMS quarterly reports. The calculation used for these reports is the MRD, or mean relative difference, which is similar to EPA's RPD except that interlaboratory, during one quarter. requires calculation of a reference detection limit, which limits may vary among samples, the MRD tile of the array of limits in the population of all duplicate is the detection limit at the 90th percenlyte during a particular quarter. The MRD is not method-specific.

\section{Method-Specific Accuracy and Precision}

The contract laboratories' EPA-approved laboratory procedures include QAVC requirements as an integral part of the methods. Thus, knowledge of the method used in obtaining data is an important component of determining data useability. EPA has conducted extensive research and the accuracy and precision of a method is available from water and waste water; information on on required QAVQC procedures. A listing of the methods used by the pions, as is full information Q procedures. A listing of the methods used by the primary laboratories during 
fourth quarter 1993 is given below along with the source for the method description. Many, if not all, of these sources include presentations of representative accuracy and precision results.

\section{Methods Used by the Contract Laboratories}

\begin{tabular}{|c|c|c|}
\hline Method & Used to Analyze & Source \\
\hline EPA120.1 & Specific conductance & EPA EMSL 1983 \\
\hline EPA150.1 & $\mathrm{pH}$ & EPA EMSL 1983 \\
\hline EPA160.1 & Total dissolved solids & EPA EMSL 1983 \\
\hline EPA160.2 & Total dissolved solids, total suspended solids & EPA EMSL 1983 \\
\hline EPA180.1 & Turbidity & EPA EMSL 1983 \\
\hline EPA200.7 & Metals & EPA EMSL 1983 \\
\hline EPA204.2 & Antimony & EPA EMSL 1983 \\
\hline EPA206.2 & Arsenic & EPA EMSL 1983 \\
\hline EPA239.2 & Lead & EPA EMSL 1983 \\
\hline EPA245.1 & Mercury & EPA EMSL 1983 \\
\hline EPA270.2 & Selenium & EPA EMSL 1983 \\
\hline EPA279.2 & Thallium & EPA EMSL 1983 \\
\hline EPA300.0 & Chloride, nitrite, sulfate & EPA EMSL 1991 \\
\hline EPA310.1 & Alkalinity & EPA EMSL 1983 \\
\hline EPA325.2 & Chloride & EPA EMSL 1983 \\
\hline EPA335.3 & Cyanide & EPA EMSL 1983 \\
\hline EPA340.2 & Fluoride & EPA EMSL 1983 \\
\hline EPA353.1 & Nitrogen, nitrate-nitrite & EPA EMSL 1983 \\
\hline EPA353.2 & Nitrogen, nitrate, nitrite, or combined & EPA EMSL 1983 \\
\hline EPA365.1 & Phosphorus, all forms (reported as total phosphates) & EPA EMSL 1983 \\
\hline EPA365.2 & Phosphorus, all forms (reported as total phosphates) & EPA EMSL 1983 \\
\hline EPA376.2 & Sulfide & EPA EMSL 1983 \\
\hline EPA413.1 & Oil \& grease & EPA EMSL 1983 \\
\hline EPA415.1 & $\begin{array}{l}\text { Dissolved organic carbon, total inorganic carbon, total } \\
\text { organic carbon }\end{array}$ & EPA EMSL 1983 \\
\hline EPA418.1 & Total petroleum hydrocarbons & EPA EMSL 1983 \\
\hline EPA420.2 & Phenols & EPA EMSL 1983 \\
\hline EPA900.0 & Gross alpha, nonvolatile beta & EPA EMSL 1980 \\
\hline EPA900.1 & Total alpha-emitting radium & EPA EMSL 1980 \\
\hline EPA906.0 & Tritium & EPA EMSL 1980 \\
\hline EPA6010 & Metals & EPA 1986 \\
\hline EPA7041 & Antimony & EPA 1986 \\
\hline EPA7060 & Arsenic & EPA 1986 \\
\hline EPA7421 & Lead & EPA 1986 \\
\hline EPA7470 & Mercury & EPA 1986 \\
\hline EPA7740 & Selenium & EPA 1986 \\
\hline EPA7841 & Thallium & EPA 1986 \\
\hline EPA8010 & Chlorinated volatile organics & $\begin{array}{l}\text { EPA } 1986 \\
\text { EPA } 1986\end{array}$ \\
\hline EPA8080 & Organochlorine pesticides and PCBs & $\begin{array}{l}\text { EPA } 1986 \\
\text { EPA } 1986\end{array}$ \\
\hline EPA8240 & GCMS volatiles & EPA 1986 \\
\hline EPA8270 & GCMS semivolatiles & EPA 1986 \\
\hline EPA8280 & Dioxins and furans & EPA 1986 \\
\hline EPA9012 & $\begin{array}{l}\text { Dioxins and furans } \\
\text { Cyanide }\end{array}$ & EPA 1986 \\
\hline EPA9020 & $\begin{array}{l}\text { Cyanide } \\
\text { Total organic halogens }\end{array}$ & EPA 1986 \\
\hline EPA9020A & $\begin{array}{l}\text { Total organic halogens } \\
\text { Total organic halogens }\end{array}$ & EPA 1986 \\
\hline EPA9030 & $\begin{array}{l}\text { Total organic halogens } \\
\text { Sulfide }\end{array}$ & EPA 1986 \\
\hline EPA9060 & $\begin{array}{l}\text { Sulfide } \\
\text { Dissolved organic carbon, total inorganic carbon, total }\end{array}$ & EPA 1986 \\
\hline & $\begin{array}{l}\text { Dissolved organic carbon, total inorganic carbon, total } \\
\text { organic carbon }\end{array}$ & EPA 1986 \\
\hline
\end{tabular}


An example of available method-specific QAQC information is that for the analysis of metals by Engineering Laboratories (GE) and Roy F. WMS, 1983). The primary laboratories, General plasma (ICP) atomic emission spectrometric method.

The following precision and accuracy data are based on the experience of seven laboratories that applied the ICP technique to acid-distilled water matrices that had been spiked with various give results for samples having three concentratories analyzed all 14 elements.) The references having the lowest values, similar to actual gration ranges; the results here are for samples

\section{ICP Precision and Accuracy Data}

\begin{tabular}{llll} 
Element & True value $(\mu \mathrm{g} / \mathrm{L})$ & $\begin{array}{l}\text { Mean reported } \\
\text { value }(\boldsymbol{\mu} / \mathrm{L})\end{array}$ & $\begin{array}{l}\text { Mean } \\
\text { Aluminum }\end{array}$ \\
\cline { 2 - 2 } & & $\underline{\text { RSD }}$ \\
Arsenic & 60 & 62 & 33 \\
Beryllium & 22 & 19 & 23 \\
Cadmium & 20 & 20 & 9.8 \\
Chromium & 2.5 & 2.9 & 16 \\
Cobalt & 10 & 10 & 18 \\
Copper & 20 & 20 & 4.1 \\
Iron & 11 & 11 & 40 \\
Lead & 20 & 19 & 15 \\
Manganese & 24 & 30 & 32 \\
Nickel & 15 & 15 & 6.7 \\
Selenium & 30 & 28 & 11 \\
Vanadium & 6 & 8.5 & 42 \\
Zinc & 70 & 69 & 2.9 \\
& 16 & 19 & 45 \\
\hline
\end{tabular}

a Relative standard deviation. In EPA (1986), the column heading is Mean Standard Deviation (\%).

As another example, EPA Method 601/8010 (EPA, 1991/EPA, 1986) is used by both GE and Weston for analyses of halogenated volatile organics. In the presentation of the method in both concentration. Contract laboratories are expecific accuracy and precision as functions of

\section{Accuracy and Precision as Functions of Concentration for EPA Method 601/8010}

Parameter

Bromodichloromethane

Bromoform

Bromomethane

Carbon tetrachloride

Chlorobenzene

Chloroethane

2-Chloroethyl vinyl ether ${ }^{f}$

Chloroform

Chloromethane

Dibromochloromethane

1,2-Dichlorobenzene

1,3-Dichlorobenzene

Accuracy as
recover $x^{\prime a}$
$1.12 C-1.02^{d}$
$0.96 C-2.05$
$0.76 C-1.27$
$0.98 C-1.04$
$1.00 C-1.23$
$0.99 C-1.53$
$1.00 C$
$0.93 C-0.39$
$0.77 C+0.18$
$0.94 C+2.72$
$0.93 C+1.70$
$0.95 C+0.43$

Single analyst

precision $(\mu \mathrm{q} / \mathrm{L})^{b}$

$0.11 \bar{X}+0.04^{e}$

$0.12 \bar{X}+0.58$

$0.28 \bar{X}+0.27$

$0.15 \bar{X}+0.38$

$0.15 \bar{X}-0.02$

$0.14 \bar{X}-0.13$

$0.20 \bar{X}$

$0.13 \bar{X}+0.15$

$0.28 \bar{X}-0.31$

$0.11 \bar{X}+1.10$

$0.20 \bar{X}+0.97$

$0.14 \bar{X}+2.33$
Overall precision $(\mu \mathrm{g} / \mathrm{L})^{c}$

$0.20 \bar{X}+1.00$

$0.21 \bar{X}+2.41$

$0.36 \bar{X}+0.94$

$0.20 \bar{X}+0.39$

$0.18 \bar{X}+1.21$

$0.17 \bar{X}+0.63$

$0.35 \bar{X}$

$0.19 \bar{X}-0.02$

$0.52 \bar{X}+1.31$

$0.24 \bar{X}+1.68$

$0.13 \bar{X}+6.13$

$0.26 \bar{X}+2.34$ 


\section{Parameter}

1,4-Dichlorobenzene

1,1-Dichloroethane

1,2-Dichloroethane

1,1-Dichloroethene

trans-1,2-Dichloroethene

Dichloromethane

(Methylene chloride)

1,2-Dichloropropane $f$

cis-1,3-Dichloropropene ${ }^{f}$

trans-1,3-Dichloropropene ${ }^{f}$

1,1,2,2-Tetrachloroethane

Tetrachloroethylene

1,1,1-Trichloroethane

1,1,2-Trichloroethane

Trichloroethylene

Trichlorofluoromethane

Vinyl chloride
Accuracy as recovery, $X^{\prime}(\mu \mathrm{g} / \mathrm{L})$

$0.93 C-0.09$

$0.95 C-1.08$

$1.04 C-1.06$

$0.98 C-0.87$

$0.97 C-0.16$

$0.91 C-0.93$

$1.00 \mathrm{C}$

$1.00 \mathrm{C}$

$1.00 \mathrm{C}$

$0.95 C+0.19$

$0.94 C+0.06$

$0.90 C-0.16$

$0.86 C+0.30$

$0.87 C+0.48$

$0.89 C-0.07$

$0.97 C-0.36$
Single analyst precision $(\mu \mathrm{g} / \mathrm{L})$

$0.15 \bar{X}+0.29$

$0.09 \bar{X}+0.17$

$0.11 \bar{X}+0.70$

$0.21 \bar{X}-0.23$

$0.11 \bar{X}+1.46$

$0.11 \bar{X}+0.33$

$0.13 \bar{X}$

$0.18 \bar{X}$

$0.18 \bar{X}$

$0.14 \bar{X}+2.41$

$0.14 \bar{X}+0.38$

$0.15 \bar{X}+0.04$

$0.13 \bar{X}-0.14$

$0.13 \bar{X}-0.03$

$0.15 \bar{X}+0.67$

$0.13 \bar{X}+0.65$
Overall precision $(\mu \mathrm{g} / \mathrm{L})$

$0.20 \bar{X}+0.41$

$0.14 \bar{X}+0.94$

$0.15 \bar{X}+0.94$

$0.29 \bar{X}-0.40$

$0.17 \bar{X}+1.46$

$0.21 \bar{X}+1.43$

$0.23 \bar{X}$

$0.32 \bar{X}$

$0.32 \bar{X}$

$0.23 \bar{X}+2.79$

$0.18 \bar{X}+2.21$

$0.20 \bar{X}+0.37$

$0.19 \bar{X}+0.67$

$0.23 \bar{X}+0.30$

$0.26 \bar{X}+0.91$

$0.27 \bar{X}+0.40$

a $\mathrm{X}^{\prime}=$ expected recovery for one or more measurements of a sample containing a concentration of $C$, in $\mu \mathrm{g} / \mathrm{L}$.

Expected single analyst standard deviation of measurements.

c Expected interlaboratory standard deviation of measurements.

$C=$ true value for the concentration, in $\mu \mathrm{g} / \mathrm{L}$.

e $\bar{X}=$ average recovery found for measurements of samples containing a concentration of $C$, in $\mu \mathrm{g} / \mathrm{L}$. Estimates based on performance of a single laboratory.

\section{References}

EPA (U.S. Environmental Protection Agency), 1986. Test Methods for Evaluating Solid Waste (SW-846), Volumes IA-IC. Washington, DC.

EPA (U.S. Environmental Protection Agency), 1991. Guidelines Establishing Test Procedures for the Analysis of Pollutants, Code of Federal Regulations, Title 40, Part 136, Appendix A. Washington, DC.

EPA EMSL (U.S. Environmental Protection Agency, Environmental Monitoring and Systems Laboratory), 1980. Prescribed Procedures for Measurement of Radioactivity in Drinking Water, EPA-600/4-80-032. Cincinnati, $\mathrm{OH}$.

EPA EMSL (U.S. Environmental Protection Agency, Environmental Monitoring and Systems Laboratory), 1983. Methods for Chemical Analysis of Water and Wastes. Cincinnati, $\mathrm{OH}$.

EPA EMSL (U.S. Environmental Protection Agency, Environmental Monitoring and Systems Laboratory), 1991. Test Method, The Determination of Inorganic Anions in Water by Ion Chromatography-Method 300.0. Cincinnati, $\mathrm{OH}$. 
WSRC-TR-94-0347

Unclassified

THIS PAGE LEFT BLANK INTENTIONALLY. 
WSRC-TR-94-0347

Unclassified

\section{Appendix F}

\section{Hydrographs}


THIS PAGE LEFT BLANK INTENTIIONALLY. 


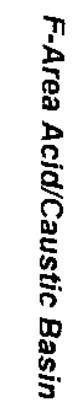

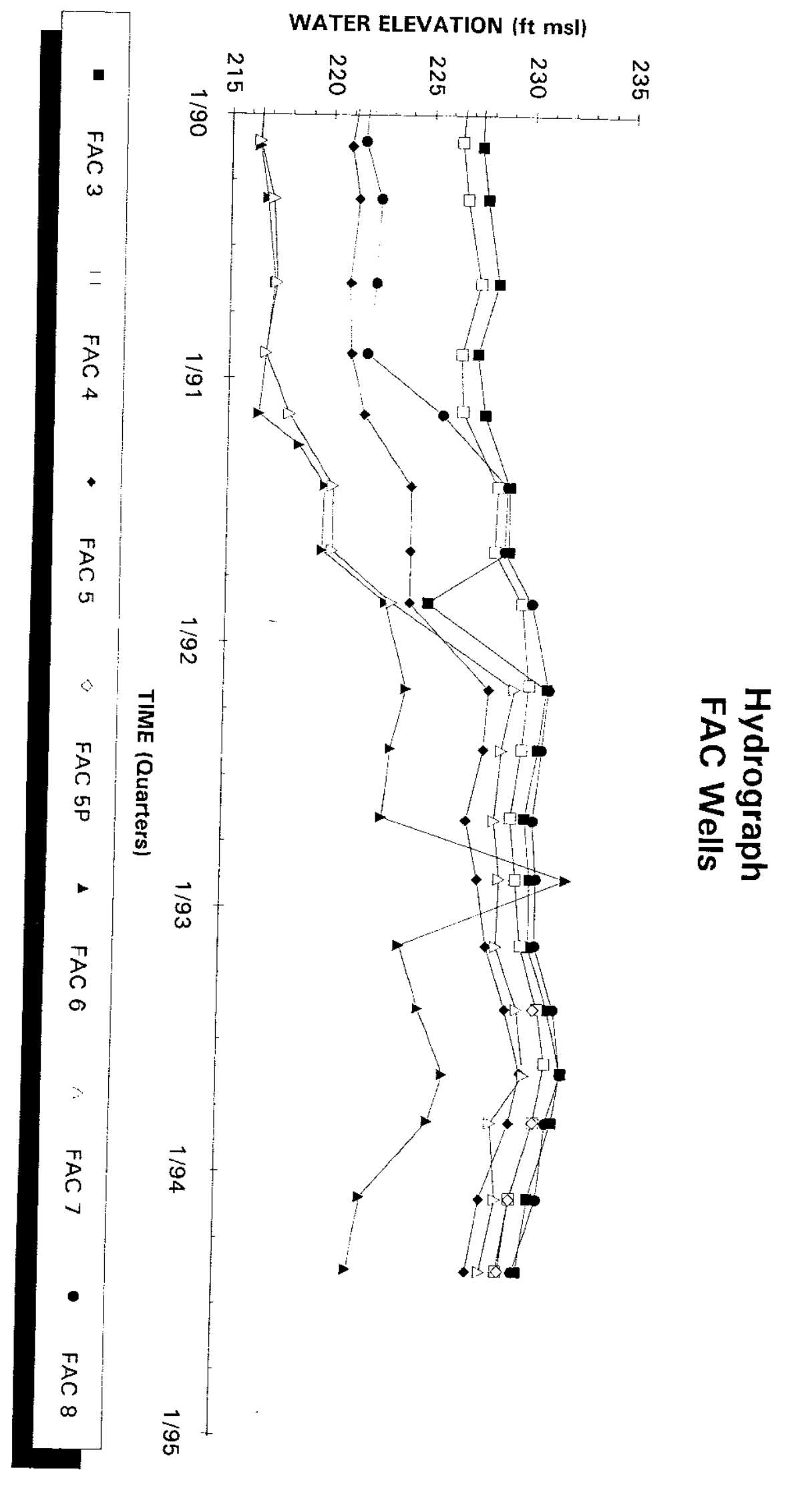

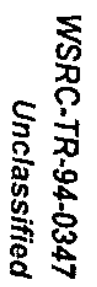


THIS PAGE LEFT BLANK INTEŃTIONALLY. 